\title{
DIFFERENTIAL OPERATORS ON QUANTIZED FLAG MANIFOLDS AT ROOTS OF UNITY, II
}

\author{
TOSHIYUKI TANISAKI
}

To Etsuro Date on his 60th birthday

\begin{abstract}
We formulate a Beilinson-Bernstein-type derived equivalence for a quantized enveloping algebra at a root of 1 as a conjecture. It says that there exists a derived equivalence between the category of modules over a quantized enveloping algebra at a root of 1 with fixed regular Harish-Chandra central character and the category of certain twisted $D$-modules on the corresponding quantized flag manifold. We show that the proof is reduced to a statement about the (derived) global sections of the ring of differential operators on the quantized flag manifold. We also give a reformulation of the conjecture in terms of the (derived) induction functor.
\end{abstract}

\section{$\S 0$. Introduction}

\section{1 .}

Let $G$ be a connected, simply connected simple algebraic group over $\mathbb{C}$, and let $H$ be a maximal torus of $G$. We denote by $\mathfrak{g}$ and $\mathfrak{h}$ the Lie algebras of $G$ and $H$, respectively. Let $Q$ and $\Lambda$ be the root lattice and the weight lattice, respectively. Let $h_{G}$ be the Coxeter number of $G$. We fix an odd integer $\ell>h_{G}$, which is prime to the order of $\Lambda / Q$ and prime to 3 if $\mathfrak{g}$ is of type $G_{2}, F_{4}, E_{6}, E_{7}, E_{8}$, and we consider the De Concini-Kac-type quantized enveloping algebra $U_{\zeta}$ at $q=\zeta=\exp (2 \pi \sqrt{-1} / \ell)$.

In [20], we started the investigation of the corresponding quantized flag manifold $\mathcal{B}_{\zeta}$, which is a noncommutative scheme, and the category of $D$-modules on it. In view of a general philosophy saying that quantized objects at roots of 1 resemble ordinary objects in positive characteristics, it

Received April 21, 2012. Revised January 28, 2013. Accepted May 14, 2013.

First published online January 7, 2014.

2010 Mathematics Subject Classification. Primary 20G05; Secondary $17 B 37$.

The author was partially supported by Grants-in-Aid for Scientific Research, Challenging Exploratory Research award 21654005 and by Grants-in-Aid for Scientific Research (C) award 24540026 from the Japan Society for the Promotion of Science.

(C) 2014 by The Editorial Board of the Nagoya Mathematical Journal 
is natural to pursue an analogue of the theory of $D$-modules on the ordinary flag manifolds in positive characteristics due to Bezrukavnikov, Mirković, and Rumynin [6]. Along this line, we have established in [20] certain Azumaya properties of the ring of differential operators on the quantized flag manifold. The aim of the present article is to investigate an analogue of another main point of [6] about the Beilinson-Bernstein-type derived equivalence.

\section{2 .}

We denote by $\mathcal{D}_{\mathcal{B}_{\zeta}, 1}$ the sheaf of rings of differential operators on the quantized flag manifold $\mathcal{B}_{\zeta}$. More generally, for each $t \in H$ we have its twisted analogue denoted by $\mathcal{D}_{\mathcal{B}_{\zeta}, t}$. It is obtained as the specialization $\mathcal{D}_{\mathcal{B}_{\zeta}} \otimes_{\mathbb{C}[H]} \mathbb{C}$ of the universally twisted sheaf $\mathcal{D}_{\mathcal{B}_{\zeta}}$ with respect to the ring homomorphism $\mathbb{C}[H] \rightarrow \mathbb{C}$ corresponding to $t \in H$.

Let $\mathcal{B}$ be the ordinary flag manifold for $G$. Then we have a Frobenius morphism $\operatorname{Fr}: \mathcal{B}_{\zeta} \rightarrow \mathcal{B}$, which is a finite morphism from a noncommutative scheme to an ordinary scheme. Taking the direct images, we obtain sheaves $\operatorname{Fr}_{*} \mathcal{D}_{\mathcal{B}_{\zeta}}, \operatorname{Fr}_{*} \mathcal{D}_{\mathcal{B}_{\zeta}, t}(t \in H)$ of rings on $\mathcal{B}$ (in the ordinary sense). Denote by $\operatorname{Mod}_{\text {coh }}\left(\operatorname{Fr}_{*} \mathcal{D}_{\mathcal{B}_{\zeta}, t}\right)$ the category of coherent $\operatorname{Fr}_{*} \mathcal{D}_{\mathcal{B}_{\zeta}, t}$-modules. Let $Z_{\mathrm{Har}}\left(U_{\zeta}\right)$ be the Harish-Chandra center of $U_{\zeta}$, and let $\mathbb{C}_{t}$ be the corresponding 1dimensional $Z_{\mathrm{Har}}\left(U_{\zeta}\right)$-module. Denote by $\operatorname{Mod}_{f}\left(U_{\zeta} \otimes_{Z_{\mathrm{Har}}\left(U_{\zeta}\right)} \mathbb{C}_{t}\right)$ the category of finitely generated $U_{\zeta} \otimes_{Z_{\mathrm{Har}}\left(U_{\zeta}\right)} \mathbb{C}_{t}$-modules. Then we have a functor

$$
R \Gamma(\mathcal{B}, \bullet): D^{b}\left(\operatorname{Mod}_{\mathrm{coh}}\left(\operatorname{Fr}_{*} \mathcal{D}_{\mathcal{B}_{\zeta}, t}\right)\right) \rightarrow D^{b}\left(\operatorname{Mod}_{f}\left(U_{\zeta} \otimes_{Z_{\mathrm{Har}}\left(U_{\zeta}\right)} \mathbb{C}_{t}\right)\right)
$$

between derived categories. It is natural in view of [6] to conjecture that (0.1) gives an equivalence if $t$ is regular. By imitating the argument of [6], we can show that this is true if we have

$$
R \Gamma\left(\mathcal{B}, \operatorname{Fr}_{*} \mathcal{D}_{\mathcal{B}_{\zeta}}\right) \cong U_{\zeta} \otimes_{Z_{\mathrm{Har}}\left(U_{\zeta}\right)} \mathbb{C}[\Lambda]
$$

However, we do not know how to prove (0.2) at present; hence, we can only state it as a conjecture. We have also a stronger conjecture,

$$
R \Gamma\left(\mathcal{B}, \operatorname{Fr}_{*}\left(\mathcal{D}_{\mathcal{B}_{\zeta}}\right)_{f}\right) \cong U_{\zeta, f} \otimes_{Z_{\mathrm{Har}}\left(U_{\zeta}\right)} \mathbb{C}[\Lambda]
$$

which is the analogue of (0.2) regarding the adjoint finite parts $\left(\mathcal{D}_{\mathcal{B}_{\zeta}}\right)_{f}, U_{\zeta, f}$ of $\mathcal{D}_{\mathcal{B}_{\zeta}}, U_{\zeta}$, respectively. We will give a reformulation of $(0.3)$ in terms of the induction functor (see Conjecture 5.2 below). It turns out that $(0.3)$ is 
equivalent to some assertions in Backelin and Kremnizer [2], [3] stated to be true under certain conditions on $\ell$ (see Remark 5.4 below).

It is also an interesting problem to find a formulation which works even in the case when the parameter $t \in H$ is singular. In the case of Lie algebras in positive characteristics, Bezrukavnikov, Mirković, and Rumynin in [5] have succeeded in giving a more general framework, which works even for singular parameters, using partial flag manifolds (quotients of $G$ by parabolic subgroups). In their case, the parameter space is $\mathfrak{h}^{*}$, and one can associate for each $h \in \mathfrak{h}^{*}$ a parabolic subgroup whose Levi subgroup is the centralizer of $h$; however, in our case the centralizer of $t \in H$ is not necessarily a Levi subgroup of a parabolic subgroup, and hence the method in [5] cannot be directly applied to our case.

\section{3 .}

This article has the following organization. In Section 1, we recall basic facts on quantized enveloping algebras at roots of 1 and the corresponding quantized flag manifolds. In Section 2, we investigate properties of the category of $D$-modules. In particular, we show that (0.2) implies (0.1) for regular $t$ and that (0.3) implies (0.2). In Sections 3 and 4, we recall some known results on the representations of quantized enveloping algebras and the induction functor, respectively. Finally, in Section 5 we give a reformulation of (0.3) in terms of the induction functor.

\section{§1. Quantized flag manifold}

\subsection{Quantized enveloping algebras}

1.1.1. Let $G$ be a connected simply connected simple algebraic group over the complex number field $\mathbb{C}$. We fix Borel subgroups $B^{+}$and $B^{-}$such that $H=B^{+} \cap B^{-}$is a maximal torus of $G$. Set $N^{+}=\left[B^{+}, B^{+}\right]$, and set $N^{-}=\left[B^{-}, B^{-}\right]$. We denote the Lie algebras of $G, B^{+}, B^{-}, H, N^{+}, N^{-}$by $\mathfrak{g}, \mathfrak{b}^{+}, \mathfrak{b}^{-}, \mathfrak{h}, \mathfrak{n}^{+}, \mathfrak{n}^{-}$, respectively. Let $\Delta \subset \mathfrak{h}^{*}$ be the root system of $(\mathfrak{g}, \mathfrak{h})$. We denote by $\Lambda \subset \mathfrak{h}^{*}$ and $Q \subset \mathfrak{h}^{*}$ the weight lattice and the root lattice, respectively. For $\lambda \in \Lambda$ we denote by $\theta_{\lambda}$ the corresponding character of $H$. The coordinate algebra $\mathbb{C}[H]$ of $H$ is naturally identified with the group algebra $\mathbb{C}[\Lambda]=\bigoplus_{\lambda \in \Lambda} \mathbb{C} e(\lambda)$ via the correspondence $\theta_{\lambda} \leftrightarrow e(\lambda)$ for $\lambda \in \Lambda$. We take a system of positive roots $\Delta^{+}$such that $\mathfrak{b}^{+}$is the sum of weight spaces with weights in $\Delta^{+} \cup\{0\}$. Let $\left\{\alpha_{i}\right\}_{i \in I}$ be the set of simple roots, and let $\left\{\varpi_{i}\right\}_{i \in I}$ be the corresponding set of fundamental weights. We denote by $\Lambda^{+}$the set of dominant integral weights. We set $Q^{+}=\bigoplus_{i \in I} \mathbb{Z}_{\geqq 0} \alpha_{i}$. Let $W \subset$ 
$G L\left(\mathfrak{h}^{*}\right)$ be the Weyl group. For $i \in I$ we denote by $s_{i} \in W$ the corresponding simple reflection. We take a $W$-invariant symmetric bilinear form

$$
(,): \mathfrak{h}^{*} \times \mathfrak{h}^{*} \rightarrow \mathbb{C}
$$

such that $(\alpha, \alpha)=2$ for short roots $\alpha$. For $\alpha \in \Delta$ we set $\alpha^{\vee}=2 \alpha /(\alpha, \alpha)$. For $i \in I$ we fix $\bar{e}_{i} \in \mathfrak{g}_{\alpha_{i}}, \bar{f}_{i} \in \mathfrak{g}_{-\alpha_{i}}$ such that $\left[\bar{e}_{i}, \bar{f}_{i}\right]=\alpha_{i}^{\vee}$ under the identification $\mathfrak{h}=\mathfrak{h}^{*}$ induced by $($,$) .$

1.1.2. For $n \in \mathbb{Z}_{\geqq 0}$ we set

$$
\begin{aligned}
{[n]_{t} } & =\frac{t^{n}-t^{-n}}{t-t^{-1}} \in \mathbb{Z}\left[t, t^{-1}\right], \\
{[n]_{t} ! } & =[n]_{t}[n-1]_{t} \cdots[2]_{t}[1]_{t} \in \mathbb{Z}\left[t, t^{-1}\right] .
\end{aligned}
$$

We denote by $U_{\mathbb{F}}$ the quantized enveloping algebra over $\mathbb{F}=\mathbb{Q}\left(q^{1 /|\Lambda / Q|}\right)$ associated to $\mathfrak{g}$. Namely, $U_{\mathbb{F}}$ is the associative algebra over $\mathbb{F}$ generated by elements

$$
k_{\lambda} \quad(\lambda \in \Lambda), \quad e_{i}, f_{i} \quad(i \in I)
$$

satisfying the relations

$$
\begin{gathered}
k_{0}=1, \quad k_{\lambda} k_{\mu}=k_{\lambda+\mu} \quad(\lambda, \mu \in \Lambda), \\
k_{\lambda} e_{i} k_{\lambda}^{-1}=q^{\left(\lambda, \alpha_{i}\right)} e_{i} \quad(\lambda \in \Lambda, i \in I), \\
k_{\lambda} f_{i} k_{\lambda}^{-1}=q^{-\left(\lambda, \alpha_{i}\right)} f_{i} \quad(\lambda \in \Lambda, i \in I), \\
e_{i} f_{j}-f_{j} e_{i}=\delta_{i j} \frac{k_{i}-k_{i}^{-1}}{q_{i}-q_{i}^{-1}} \quad(i, j \in I), \\
\sum_{n=0}^{1-a_{i j}}(-1)^{n} e_{i}^{\left(1-a_{i j}-n\right)} e_{j} e_{i}^{(n)}=0 \quad(i, j \in I, i \neq j), \\
\sum_{n=0}^{1-a_{i j}}(-1)^{n} f_{i}^{\left(1-a_{i j}-n\right)} f_{j} f_{i}^{(n)}=0 \quad(i, j \in I, i \neq j),
\end{gathered}
$$

where $q_{i}=q^{\left(\alpha_{i}, \alpha_{i}\right) / 2}, k_{i}=k_{\alpha_{i}}, a_{i j}=2\left(\alpha_{i}, \alpha_{j}\right) /\left(\alpha_{i}, \alpha_{i}\right)$ for $i, j \in I$, and

$$
e_{i}^{(n)}=e_{i}^{n} /[n]_{q_{i}} !, \quad f_{i}^{(n)}=f_{i}^{n} /[n]_{q_{i}} !
$$


for $i \in I$ and $n \in \mathbb{Z}_{\geqq 0}$. We will use the Hopf algebra structure of $U_{\mathbb{F}}$ given by

$$
\begin{aligned}
& \Delta\left(k_{\lambda}\right)=k_{\lambda} \otimes k_{\lambda} \quad(\lambda \in \Lambda), \\
& \Delta\left(e_{i}\right)=e_{i} \otimes 1+k_{i} \otimes e_{i}, \quad \Delta\left(f_{i}\right)=f_{i} \otimes k_{i}^{-1}+1 \otimes f_{i} \quad(i \in I), \\
& \varepsilon\left(k_{\lambda}\right)=1, \quad \varepsilon\left(e_{i}\right)=\varepsilon\left(f_{i}\right)=0 \quad(\lambda \in \Lambda, i \in I), \\
& S\left(k_{\lambda}\right)=k_{\lambda}^{-1}, \quad S\left(e_{i}\right)=-k_{i}^{-1} e_{i}, \quad S\left(f_{i}\right)=-f_{i} k_{i} \quad(\lambda \in \Lambda, i \in I) .
\end{aligned}
$$

Define subalgebras $U_{\mathbb{F}}^{0}, U_{\mathbb{F}}^{+}, U_{\mathbb{F}}^{-}, U_{\mathbb{F}}^{\geqq 0}, U_{\mathbb{F}}^{\leqq 0}$ of $U_{\mathbb{F}}$ by

$$
\begin{gathered}
U_{\mathbb{F}}^{0}=\left\langle k_{\lambda} \mid \lambda \in \Lambda\right\rangle, \quad U_{\mathbb{F}}^{+}=\left\langle e_{i} \mid i \in I\right\rangle, \quad U_{\mathbb{F}}^{-}=\left\langle f_{i} \mid i \in I\right\rangle, \\
U_{\mathbb{F}}^{\geqq 0}=\left\langle k_{\lambda}, e_{i} \mid \lambda \in \Lambda, i \in I\right\rangle, \quad U_{\mathbb{F}}^{\leqq 0}=\left\langle k_{\lambda}, f_{i} \mid \lambda \in \Lambda, i \in I\right\rangle .
\end{gathered}
$$

The multiplication of $U_{\mathbb{F}}$ induces isomorphisms

$$
\begin{aligned}
& U_{\mathbb{F}} \cong U_{\mathbb{F}}^{-} \otimes U_{\mathbb{F}}^{0} \otimes U_{\mathbb{F}}^{+} \cong U_{\mathbb{F}}^{+} \otimes U_{\mathbb{F}}^{0} \otimes U_{\mathbb{F}}^{-}, \\
& U_{\mathbb{F}}^{\geqq 0} \cong U_{\mathbb{F}}^{0} \otimes U_{\mathbb{F}}^{+} \cong U_{\mathbb{F}}^{+} \otimes U_{\mathbb{F}}^{0}, \\
& U_{\mathbb{F}}^{\leqq 0} \cong U_{\mathbb{F}}^{0} \otimes U_{\mathbb{F}}^{-} \cong U_{\mathbb{F}}^{-} \otimes U_{\mathbb{F}}^{0},
\end{aligned}
$$

of $\mathbb{F}$-modules. The fact $(1.1)$ is called the triangular decomposition of $U_{\mathbb{F}}$. For $\gamma \in Q$ we set

$$
U_{\mathbb{F}, \gamma}^{ \pm}=\left\{u \in U_{\mathbb{F}}^{ \pm} \mid k_{\mu} u k_{-\mu}=q^{(\gamma, \mu)} u(\mu \in \Lambda)\right\}
$$

Then we have

$$
U_{\mathbb{F}}^{ \pm}=\bigoplus_{\gamma \in Q^{+}} U_{\mathbb{F}, \pm \gamma}^{ \pm} .
$$

For $i \in I$ we denote by $T_{i}$ the automorphism of the algebra $U_{\mathbb{F}}$ given by

$$
\begin{aligned}
& T_{i}\left(k_{\mu}\right)=k_{s_{i} \mu}(\mu \in \Lambda), \\
& T_{i}\left(e_{j}\right)= \begin{cases}\sum_{k=0}^{-a_{i j}}(-1)^{k} q_{i}^{-k} e_{i}^{\left(-a_{i j}-k\right)} e_{j} e_{i}^{(k)} & (j \in I, j \neq i), \\
-f_{i} k_{i} & (j=i),\end{cases} \\
& T_{i}\left(f_{j}\right)= \begin{cases}\sum_{k=0}^{-a_{i j}}(-1)^{k} q_{i}^{k} f_{i}^{(k)} f_{j} f_{i}^{\left(-a_{i j}-k\right)} & (j \in I, j \neq i), \\
-k_{i}^{-1} e_{i} & (j=i)\end{cases}
\end{aligned}
$$


(see [15]). Let $w_{0}$ be the longest element of $W$. We fix a reduced expression

$$
w_{0}=s_{i_{1}} \cdots s_{i_{N}}
$$

of $w_{0}$, and we set

$$
\beta_{k}=s_{i_{1}} \cdots s_{i_{k-1}}\left(\alpha_{i_{k}}\right) \quad(1 \leqq k \leqq N) .
$$

Then we have $\Delta^{+}=\left\{\beta_{k} \mid 1 \leqq k \leqq N\right\}$. For $1 \leqq k \leqq N$ we set

$$
e_{\beta_{k}}=T_{i_{1}} \cdots T_{i_{k-1}}\left(e_{i_{k}}\right), \quad f_{\beta_{k}}=T_{i_{1}} \cdots T_{i_{k-1}}\left(f_{i_{k}}\right) .
$$

Then $\left\{e_{\beta_{N}}^{m_{N}} \cdots e_{\beta_{1}}^{m_{1}} \mid m_{1}, \ldots, m_{N} \geqq 0\right\}$ (resp., $\left\{f_{\beta_{N}}^{m_{N}} \cdots f_{\beta_{1}}^{m_{1}} \mid m_{1}, \ldots, m_{N} \geqq\right.$ $0\}$ ) is an $\mathbb{F}$-basis of $U_{\mathbb{F}}^{+}$(resp., $U_{\mathbb{F}}^{-}$), called the PBW (Poincaré-BirkhoffWitt) basis (see [14]). We have $e_{\alpha_{i}}=e_{i}$ and $f_{\alpha_{i}}=f_{i}$ for any $i \in I$.

Denote by

$$
\tau: U_{\mathbb{F}}^{\geqq 0} \times U_{\mathbb{F}}^{\leqq 0} \rightarrow \mathbb{F}
$$

the Drinfeld pairing. It is characterized as the unique bilinear form satisfying

$$
\begin{aligned}
\tau\left(x, y_{1} y_{2}\right) & =(\tau \otimes \tau)\left(\Delta(x), y_{1} \otimes y_{2}\right) \quad\left(x \in U_{\mathbb{F}}^{\geqq 0}, y_{1}, y_{2} \in U_{\mathbb{F}}^{\leqq 0}\right), \\
\tau\left(x_{1} x_{2}, y\right) & =(\tau \otimes \tau)\left(x_{2} \otimes x_{1}, \Delta(y)\right) \quad\left(x_{1}, x_{2} \in U_{\mathbb{F}}^{\geqq 0}, y \in U_{\mathbb{F}}^{\leqq 0}\right), \\
\tau\left(k_{\lambda}, k_{\mu}\right) & =q^{-(\lambda, \mu)} \quad(\lambda, \mu \in \Lambda), \\
\tau\left(k_{\lambda}, f_{i}\right) & =\tau\left(e_{i}, k_{\lambda}\right)=0 \quad(\lambda \in \Lambda, i \in I), \\
\tau\left(e_{i}, f_{j}\right) & =\delta_{i j} /\left(q_{i}^{-1}-q_{i}\right) \quad(i, j \in I)
\end{aligned}
$$

(see [15], [18]). It also satisfies the following.

Lemma 1.1 ([15, Section 1.2], [18, Proposition 2.1.1]). We have the following:

(i) $\quad \tau(S(x), S(y))=\tau(x, y)$ for $x \in U_{\mathbb{F}}^{\geqq 0}, y \in U_{\mathbb{F}}^{\leqq 0}$;

(ii) for $x \in U_{\mathbb{F}}^{\geqq 0}, y \in U_{\mathbb{F}}^{\leqq 0}$ we have

$$
\begin{aligned}
& y x=\sum_{(x)_{2},(y)_{2}} \tau\left(x_{(0)}, S\left(y_{(0)}\right)\right) \tau\left(x_{(2)}, y_{(2)}\right) x_{(1)} y_{(1)}, \\
& x y=\sum_{(x)_{2},(y)_{2}} \tau\left(x_{(0)}, y_{(0)}\right) \tau\left(x_{(2)}, S\left(y_{(2)}\right)\right) y_{(1)} x_{(1)}
\end{aligned}
$$


(iii) $\tau\left(x k_{\lambda}, y k_{\mu}\right)=q^{-(\lambda, \mu)} \tau(x, y)$ for $\lambda, \mu \in \Lambda, x \in U_{\mathbb{F}}^{+}, y \in U_{\mathbb{F}}^{-}$;

(iv) $\tau\left(U_{\mathbb{F}, \beta}^{+}, U_{\mathbb{F},-\gamma}^{-}\right)=\{0\}$ for $\beta, \gamma \in Q^{+}$with $\beta \neq \gamma$;

(v) for any $\beta \in Q^{+}$, the restriction $\left.\tau\right|_{U_{\mathbb{F}, \beta}^{+} \times U_{\mathbb{F},-\beta}^{-}}$is nondegenerate.

We define an algebra homomorphism

$$
\operatorname{ad}: U_{\mathbb{F}} \rightarrow \operatorname{End}_{\mathbb{F}}\left(U_{\mathbb{F}}\right)
$$

by

$$
\operatorname{ad}(u)(v)=\sum_{(u)} u_{(0)} v\left(S u_{(1)}\right) \quad\left(u, v \in U_{\mathbb{F}}\right) .
$$

1.1.3. We fix an integer $\ell>1$ satisfying

(a) $\ell$ is odd;

(b) $\ell$ is prime to 3 if $G$ is of type $G_{2}, F_{4}, E_{6}, E_{7}, E_{8}$;

(c) $\ell$ is prime to $|\Lambda / Q|$;

and a primitive $\ell$ th root $\zeta^{\prime} \in \mathbb{C}$ of 1 . Define a subring $\mathbb{A}$ of $\mathbb{F}$ by

$$
\mathbb{A}=\left\{f\left(q^{1 /|\Lambda / Q|}\right) \mid f(x) \in \mathbb{Q}(x), f \text { is regular at } x=\zeta^{\prime}\right\}
$$

We set $\zeta=\left(\zeta^{\prime}\right)^{|\Lambda / Q|}$. We note that $\zeta$ is also a primitive $\ell$ th root of 1 by condition (c).

We denote by $U_{\mathbb{A}}^{L}, U_{\mathbb{A}}$ the $\mathbb{A}$-forms of $U_{\mathbb{F}}$ called the Lusztig form and the De Concini-Kac form, respectively. Namely, we have

$$
\begin{aligned}
& U_{\mathbb{A}}^{L}=\left\langle e_{i}^{(m)}, f_{i}^{(m)}, k_{\lambda} \mid i \in I, m \in \mathbb{Z}_{\geqq 0}, \lambda \in \Lambda\right\rangle_{\mathbb{A} \text {-alg }} \subset U_{\mathbb{F}}, \\
& U_{\mathbb{A}}=\left\langle e_{i}, f_{i}, k_{\lambda} \mid i \in I, \lambda \in \Lambda\right\rangle_{\mathbb{A} \text {-alg }} \subset U_{\mathbb{F}} .
\end{aligned}
$$

We have obviously $U_{\mathbb{A}} \subset U_{\mathbb{A}}^{L}$. The Hopf algebra structure of $U_{\mathbb{F}}$ induces Hopf algebra structures over $\mathbb{A}$ of $U_{\mathbb{A}}^{L}$ and $U_{\mathbb{A}}$. We set

$$
\begin{gathered}
U_{\mathbb{A}}^{L, b}=U_{\mathbb{A}}^{L} \cap U_{\mathbb{F}}^{b}, \quad U_{\mathbb{A}}^{b}=U_{\mathbb{A}} \cap U_{\mathbb{F}}^{b} \quad(b=+,-, 0, \geqq 0, \leqq 0), \\
U_{\mathbb{A}, \pm \gamma}^{L, \pm}=U_{\mathbb{A}}^{L} \cap U_{\mathbb{F}, \pm \gamma}^{ \pm}, \quad U_{\mathbb{A}, \pm \gamma}^{ \pm}=U_{\mathbb{A}} \cap U_{\mathbb{F}, \pm \gamma}^{ \pm} \quad\left(\gamma \in Q^{+}\right) .
\end{gathered}
$$

Then we have triangular decompositions

$$
\begin{aligned}
& U_{\mathbb{A}}^{L} \cong U_{\mathbb{A}}^{L,-} \otimes_{\mathbb{A}} U_{\mathbb{A}}^{L, 0} \otimes_{\mathbb{A}} U_{\mathbb{A}}^{L,+}, \\
& U_{\mathbb{A}} \cong U_{\mathbb{A}}^{-} \otimes_{\mathbb{A}} U_{\mathbb{A}}^{0} \otimes_{\mathbb{A}} U_{\mathbb{A}}^{+} .
\end{aligned}
$$


Moreover, we have

$$
U_{\mathbb{A}}^{L, \pm}=\bigoplus_{\gamma \in Q^{+}} U_{\mathbb{A}, \pm \gamma}^{L, \pm}, \quad U_{\mathbb{A}}^{ \pm}=\bigoplus_{\gamma \in Q^{+}} U_{\mathbb{A}, \pm \gamma}^{ \pm} .
$$

The Drinfeld pairing (1.4) induces

$$
L_{\tau_{\mathbb{A}}}: U_{\mathbb{A}}^{L, \geqq 0} \times U_{\mathbb{A}}^{\leqq 0} \rightarrow \mathbb{A}, \quad \tau_{\mathbb{A}}^{L}: U_{\mathbb{A}}^{\geqq 0} \times U_{\mathbb{A}}^{L, \leqq 0} \rightarrow \mathbb{A} .
$$

LEMmA 1.2. We have $\operatorname{ad}\left(U_{\mathbb{A}}^{L}\right)\left(U_{\mathbb{A}}\right) \subset U_{\mathbb{A}}$.

Proof. It is sufficient to show that

$$
\begin{aligned}
\operatorname{ad}\left(k_{\lambda}\right)\left(U_{\mathbb{A}}\right) \subset U_{\mathbb{A}} & (\lambda \in \Lambda), \\
\operatorname{ad}\left(e_{i}^{(n)}\right)\left(U_{\mathbb{A}}\right) \subset U_{\mathbb{A}} & \left(i \in I, n \in \mathbb{Z}_{\geqq 0}\right), \\
\operatorname{ad}\left(f_{i}^{(n)}\right)\left(U_{\mathbb{A}}\right) \subset U_{\mathbb{A}} & \left(i \in I, n \in \mathbb{Z}_{\geqq 0}\right) .
\end{aligned}
$$

The proof of (1.6) is easy and omitted. By the formulas

$$
\begin{aligned}
& \operatorname{ad}(x)(u v)=\sum_{(x)} \operatorname{ad}\left(x_{(0)}\right)(u) \operatorname{ad}\left(x_{(1)}\right)(v) \quad\left(x \in U_{\mathbb{A}}^{L}, u, v \in U_{\mathbb{A}}\right), \\
& \Delta\left(e_{i}^{(n)}\right)=\sum_{r=0}^{n} q_{i}^{r(n-r)} e_{i}^{(n-r)} k_{i}^{r} \otimes e_{i}^{(r)} \quad(i \in I, n \geqq 0), \\
& \Delta\left(f_{i}^{(n)}\right)=\sum_{r=0}^{n} q_{i}^{-r(n-r)} f_{i}^{(r)} \otimes k_{i}^{-r} f_{i}^{(n-r)} \quad(i \in I, n \geqq 0),
\end{aligned}
$$

we have only to show that

$$
\begin{array}{ll}
\operatorname{ad}\left(e_{i}^{(n)}\right)(u) \in U_{\mathbb{A}} & \left(i \in I, n \in \mathbb{Z}_{\geqq 0}, u=k_{\lambda}, e_{j}, f_{j} k_{j}\right), \\
\operatorname{ad}\left(f_{i}^{(n)}\right)(u) \in U_{\mathbb{A}} & \left(i \in I, n \in \mathbb{Z}_{\geqq 0}, u=k_{\lambda}, e_{j}, f_{j}\right) .
\end{array}
$$

For $\lambda \in \Lambda, i, j \in I$ with $i \neq j$ and $n \in \mathbb{Z}_{>0}$, we have

$$
\begin{aligned}
& \operatorname{ad}\left(e_{i}^{(n)}\right)\left(k_{\lambda}\right)=\frac{(-1)^{n} q_{i}^{n(n-1)}}{[n]_{q_{i}} !}\left(\prod_{j=0}^{n-1}\left(q_{i}^{\left(\lambda, \alpha_{i}^{\vee}\right)}-q_{i}^{-2 j}\right)\right) e_{i}^{n} k_{\lambda} \\
& \operatorname{ad}\left(e_{i}^{(n)}\right)\left(e_{i}\right)=q_{i}^{-n(n+1) / 2}\left(q_{i}-q_{i}^{-1}\right)^{n} e_{i}^{n+1}
\end{aligned}
$$




$$
\begin{aligned}
\operatorname{ad}\left(e_{i}^{(n)}\right)\left(e_{j}\right) & = \begin{cases}\sum_{r=0}^{n}(-1)^{r} q_{i}^{r\left(n-1+a_{i j}\right)} e_{i}^{(n-r)} e_{j} e_{i}^{(r)} & \left(n<1-a_{i j}\right), \\
0 & \left(n \geqq 1-a_{i j}\right),\end{cases} \\
\operatorname{ad}\left(e_{i}^{(n)}\right)\left(f_{i} k_{i}\right) & = \begin{cases}\left(k_{i}^{2}-1\right) /\left(q_{i}-q_{i}^{-1}\right) & (n=1), \\
(-1)^{n-1} q_{i}^{(n-1)(n+2) / 2}\left(q_{i}-q_{i}^{-1}\right)^{n-2} e_{i}^{n-1} k_{i}^{2} & (n>1),\end{cases} \\
\operatorname{ad}\left(e_{i}^{(n)}\right)\left(f_{j} k_{j}\right) & =0,
\end{aligned}
$$

and hence (1.9) holds. (Note that $[r]_{q_{i}}$ ! is invertible in $\mathbb{A}$ for $r \leqq-a_{i j}$.) The proof of (1.10) is similar and omitted.

1.1.4. Let us consider the specialization

$$
\mathbb{A} \rightarrow \mathbb{C} \quad\left(q^{1 /|\Lambda / Q|} \mapsto \zeta^{\prime}\right) .
$$

Note that $q$ is mapped to $\zeta=\left(\zeta^{\prime}\right)^{|\Lambda / Q|} \in \mathbb{C}$, which is also a primitive $\ell$ th root of 1 . We set

$$
\begin{aligned}
U_{\zeta}^{L} & =\mathbb{C} \otimes_{\mathbb{A}} U_{\mathbb{A}}^{L}, \quad U_{\zeta}=\mathbb{C} \otimes_{\mathbb{A}} U_{\mathbb{A}}, \\
U_{\zeta}^{L, b} & =\mathbb{C} \otimes_{\mathbb{A}} U_{\mathbb{A}}^{L, b}, \quad U_{\zeta}^{b}=\mathbb{C} \otimes_{\mathbb{A}} U_{\mathbb{A}}^{b} \quad(b=+,-, 0, \geqq 0, \leqq 0), \\
U_{\zeta, \pm \gamma}^{L, \pm} & =\mathbb{C} \otimes_{\mathbb{A}} U_{\mathbb{A}, \pm \gamma}^{L, \pm}, \quad U_{\zeta, \pm \gamma}^{ \pm}=\mathbb{C} \otimes_{\mathbb{A}} U_{\mathbb{A}, \pm \gamma}^{ \pm} \quad\left(\gamma \in Q^{+}\right) .
\end{aligned}
$$

Then $U_{\zeta}^{L}$ and $U_{\zeta}$ are Hopf algebras over $\mathbb{C}$, and we have triangular decompositions

$$
\begin{aligned}
& U_{\zeta}^{L} \cong U_{\zeta}^{L,-} \otimes U_{\zeta}^{L, 0} \otimes U_{\zeta}^{L,+}, \\
& U_{\zeta} \cong U_{\zeta}^{-} \otimes U_{\zeta}^{0} \otimes U_{\zeta}^{+} .
\end{aligned}
$$

Moreover, we have

$$
U_{\zeta}^{L, \pm}=\bigoplus_{\gamma \in Q^{+}} U_{\zeta, \pm \gamma}^{L, \pm}, \quad U_{\zeta}^{ \pm}=\bigoplus_{\gamma \in Q^{+}} U_{\zeta, \pm \gamma}^{ \pm} .
$$

By De Concini and Kac [8, Proposition 1.7], we have the following.

LEMMA 1.3.

(i) The set $\left\{e_{\beta_{N}}^{m_{N}} \cdots e_{\beta_{1}}^{m_{1}} \mid m_{1}, \ldots, m_{N} \geqq 0\right\}$ (resp., $\left\{f_{\beta_{N}}^{m_{N}} \cdots f_{\beta_{1}}^{m_{1}} \mid m_{1}, \ldots\right.$, $\left.m_{N} \geqq 0\right\}$ ) forms a $\mathbb{C}$-basis of $U_{\zeta}^{+}$(resp., $U_{\zeta}^{-}$).

(ii) The set $\left\{k_{\lambda} \mid \lambda \in \Lambda\right\}$ forms a $\mathbb{C}$-basis of $U_{\zeta}^{0}$. 
The Drinfeld pairings (1.5) induce

$$
{ }^{L} \tau_{\zeta}: U_{\zeta}^{L, \geqq 0} \times U_{\zeta}^{\leqq 0} \rightarrow \mathbb{C}, \quad \tau_{\zeta}^{L}: U_{\zeta}^{\geqq 0} \times U_{\zeta}^{L, \leqq 0} \rightarrow \mathbb{C} .
$$

Moreover, we have the following (see [20, Lemma 1.5]).

Proposition 1.4. For any $\gamma \in Q^{+}$, the restrictions of ${ }^{L} \tau_{\zeta}$ and $\tau_{\zeta}^{L}$ to

$$
U_{\zeta, \gamma}^{L,+} \times U_{\zeta,-\gamma}^{-} \rightarrow \mathbb{C}, \quad U_{\zeta, \gamma}^{-} \times U_{\zeta,-\gamma}^{L,-} \rightarrow \mathbb{C},
$$

respectively, are nondegenerate.

By Lemma 1.2 we have an algebra homomorphism

$$
\operatorname{ad}: U_{\zeta}^{L} \rightarrow \operatorname{End}_{\mathbb{C}}\left(U_{\zeta}\right)
$$

In general, for a Lie algebra $\mathfrak{s}$ we denote its enveloping algebra by $U(\mathfrak{s})$. We denote by

$$
\pi: U_{\zeta}^{L} \rightarrow U(\mathfrak{g})
$$

Lusztig's Frobenius homomorphism (see [14]). Namely, $\pi$ is the $\mathbb{C}$-algebra homomorphism given by

$$
\pi\left(e_{i}^{(m)}\right)=\left\{\begin{array}{ll}
\bar{e}_{i}^{(m / \ell)} & (\ell \mid m) \\
0 & (\ell / m),
\end{array} \quad \pi\left(f_{i}^{(m)}\right)=\left\{\begin{array}{ll}
\bar{f}_{i}^{(m / \ell)} & (\ell \mid m) \\
0 & (\ell \not / m),
\end{array} \quad \pi\left(k_{\lambda}\right)=1\right.\right.
$$

for $i \in I, m \in \mathbb{Z}_{\geqq 0}, \lambda \in \Lambda$. Here, $\bar{e}_{i}^{(n)}=\bar{e}_{i}^{n} / n$ !, $\bar{f}_{i}^{(n)}=\bar{f}_{i}^{n} / n$ ! for $i \in I$ and $n \in \mathbb{Z}_{\geqq 0}$. Then $\pi$ is a homomorphism of Hopf algebras.

We recall the description of the center $Z\left(U_{\zeta}\right)$ of the algebra $U_{\zeta}$ due to De Concini and Kac [8, Section 3] and De Concini and Procesi [9, Section 21]. Denote by $Z\left(U_{\mathbb{F}}\right)$ the center of $U_{\mathbb{F}}$, and define a subalgebra $Z_{\mathrm{Har}}\left(U_{\zeta}\right)$ of $Z\left(U_{\zeta}\right)$ by

$$
Z_{\mathrm{Har}}\left(U_{\zeta}\right)=\operatorname{Im}\left(Z\left(U_{\mathbb{F}}\right) \cap U_{\mathbb{A}} \rightarrow U_{\zeta}\right)
$$

We define a shifted action of $W$ on the group algebra $\mathbb{C}[\Lambda]=\bigoplus_{\lambda \in \Lambda} \mathbb{C} e(\lambda)$ of $\Lambda$ by

$$
w \circ e(\lambda)=\zeta^{(w \lambda-\lambda, \rho)} e(w \lambda) \quad(w \in W, \lambda \in \Lambda)
$$

Let

$$
\iota: Z_{\mathrm{Har}}\left(U_{\zeta}\right) \rightarrow \mathbb{C}[\Lambda]
$$


be the composite of

$$
Z_{\mathrm{Har}}\left(U_{\zeta}\right) \hookrightarrow U_{\zeta} \cong U_{\zeta}^{-} \otimes U_{\zeta}^{0} \otimes U_{\zeta}^{+} \stackrel{\varepsilon \otimes 1 \otimes \varepsilon}{\longrightarrow} U_{\zeta}^{0} \cong \mathbb{C}[\Lambda],
$$

where $U_{\zeta}^{0} \cong \mathbb{C}[\Lambda]$ is given by $k_{\lambda} \leftrightarrow e(\lambda)$. Then by [8, Lemma 3.9], $\iota$ is an injective algebra homomorphism with image

$$
\mathbb{C}[2 \Lambda]^{W \circ}=\{f \in \mathbb{C}[2 \Lambda] \mid w \circ f=f(\forall w \in W)\} .
$$

In particular, we have an isomorphism

$$
Z_{\mathrm{Har}}\left(U_{\zeta}\right) \simeq \mathbb{C}[2 \Lambda]^{W \circ}
$$

of $\mathbb{C}$-algebras. By $[8$, Section 3.1$]$ the elements

$$
e_{\beta}^{\ell}, \quad f_{\beta}^{\ell}, \quad k_{\ell \lambda} \quad\left(\beta \in \Delta^{+}, \lambda \in \Lambda\right)
$$

are central in $U_{\zeta}$. Let $Z_{\mathrm{Fr}}\left(U_{\zeta}\right)$ be the subalgebra of $U_{\zeta}$ generated by them. It is a Hopf subalgebra of $U_{\zeta}$. Define an algebraic subgroup $K$ of $B^{+} \times B^{-}$ by

$$
K=\left\{\left(g h, g^{\prime} h^{-1}\right) \mid h \in H, g \in N^{+}, g^{\prime} \in N^{-}\right\} .
$$

By [9, Section 19.1] we have an isomorphism

$$
Z_{\mathrm{Fr}}\left(U_{\zeta}\right) \cong \mathbb{C}[K]
$$

of Hopf algebras (see also [10, Theorem 7.4]). We refer the reader to [20, Section 1.5] for the explicit description of the isomorphism (1.16). By [9], $Z\left(U_{\zeta}\right)$ is generated by $Z_{\mathrm{Fr}}\left(U_{\zeta}\right)$ and $Z_{\mathrm{Har}}\left(U_{\zeta}\right)$. Moreover, we have an isomorphism

$$
Z\left(U_{\zeta}\right) \cong Z_{\mathrm{Har}}\left(U_{\zeta}\right) \otimes_{Z_{\mathrm{Har}}\left(U_{\zeta}\right) \cap Z_{\mathrm{Fr}}\left(U_{\zeta}\right)} Z_{\mathrm{Fr}}\left(U_{\zeta}\right) \quad\left(z_{1} z_{2} \leftrightarrow z_{1} \otimes z_{2}\right)
$$

of algebras.

\subsection{Sheaves on quantized flag manifolds}

1.2.1. We denote by $C_{\mathbb{F}}$ the subspace of $U_{\mathbb{F}}^{*}=\operatorname{Hom}_{\mathbb{F}}\left(U_{\mathbb{F}}, \mathbb{F}\right)$ spanned by the matrix coefficients of finite-dimensional $U_{\mathbb{F}}$-modules of type 1 in the sense of Lusztig, and we denote by

$$
\langle,\rangle: C_{\mathbb{F}} \times U_{\mathbb{F}} \rightarrow \mathbb{F}
$$

the canonical pairing. Then $C_{\mathbb{F}}$ is endowed with a Hopf algebra structure dual to $U_{\mathbb{F}}$ via $(1.17)$. We have a $U_{\mathbb{F}}$-bimodule structure of $C_{\mathbb{F}}$ given by

$$
\left\langle u_{1} \cdot \varphi \cdot u_{2}, u\right\rangle=\left\langle\varphi, u_{2} u u_{1}\right\rangle \quad\left(\varphi \in C_{\mathbb{F}}, u, u_{1}, u_{2} \in U_{\mathbb{F}}\right) .
$$


Define a $\Lambda$-graded ring $A_{\mathbb{F}}=\bigoplus_{\lambda \in \Lambda^{+}} A_{\mathbb{F}}(\lambda)$ by

$$
\begin{aligned}
A_{\mathbb{F}} & =\left\{\varphi \in C_{\mathbb{F}} \mid \varphi \cdot f_{i}=0(i \in I)\right\}, \\
A_{\mathbb{F}}(\lambda) & =\left\{\varphi \in A_{\mathbb{F}} \mid \varphi \cdot k_{\mu}=q^{(\mu, \lambda)} \varphi(\mu \in \Lambda)\right\} .
\end{aligned}
$$

Note that $A_{\mathbb{F}}$ is a left $U_{\mathbb{F}^{-}}$-submodule of $C_{\mathbb{F}}$. For $\lambda \in \Lambda^{+}$and $\xi \in \Lambda$, we set

$$
A_{\mathbb{F}}(\lambda)_{\xi}=\left\{\varphi \in A_{\mathbb{F}}(\lambda) \mid k_{\mu} \cdot \varphi=q^{(\xi, \mu)} \varphi\right\}
$$

Then we have

$$
A_{\mathbb{F}}(\lambda)=\bigoplus_{\xi \in \lambda-Q^{+}} A_{\mathbb{F}}(\lambda)_{\xi} .
$$

We define $\mathbb{A}$-forms $C_{\mathbb{A}}, A_{\mathbb{A}}, A_{\mathbb{A}}(\lambda)\left(\lambda \in \Lambda^{+}\right)$of $C_{\mathbb{F}}, A_{\mathbb{F}}, A_{\mathbb{F}}(\lambda)$, respectively, by

$$
C_{\mathbb{A}}=\left\{\varphi \in C_{\mathbb{F}} \mid\left\langle\varphi, U_{\mathbb{A}}^{L}\right\rangle \subset \mathbb{A}\right\}, \quad A_{\mathbb{A}}=A_{\mathbb{F}} \cap C_{\mathbb{A}}, \quad A_{\mathbb{A}}(\lambda)=A_{\mathbb{F}}(\lambda) \cap C_{\mathbb{A}}
$$

Then $C_{\mathbb{A}}$ is a Hopf algebra over $\mathbb{A}$, and $A_{\mathbb{A}}$ is its $\mathbb{A}$-subalgebra. Moreover, $C_{\mathbb{A}}$ is a $U_{\mathbb{A}}^{L}$-bimodule, and $A_{\mathbb{A}}$ is its left $U_{\mathbb{A}}^{L}$-submodule. We also set $A_{\mathbb{A}}(\lambda)_{\xi}=$ $A_{\mathbb{F}}(\lambda)_{\xi} \cap A_{\mathbb{A}}$ for $\lambda \in \Lambda^{+}, \xi \in \Lambda$.

We set

$$
C_{\zeta}=\mathbb{C} \otimes_{\mathbb{A}} C_{\mathbb{A}}, \quad A_{\zeta}=\mathbb{C} \otimes_{\mathbb{A}} A_{\mathbb{A}}, \quad A_{\zeta}(\lambda)=\mathbb{C} \otimes_{\mathbb{A}} A_{\mathbb{A}}(\lambda) \quad\left(\lambda \in \Lambda^{+}\right) .
$$

Then $C_{\zeta}$ is a Hopf algebra over $\mathbb{C}$. Moreover, the $U_{\mathbb{F}}$-bimodule structure of $C_{\mathbb{F}}$ induces a $U_{\zeta}^{L}$-bimodule structure of $C_{\zeta}$. For $\lambda \in \Lambda^{+}$and $\xi \in \Lambda$, we set $A_{\zeta}(\lambda)_{\xi}=\mathbb{C} \otimes_{\mathbb{A}} A_{\mathbb{A}}(\lambda)_{\xi}$. Then we have

$$
A_{\zeta}(\lambda)=\bigoplus_{\xi \in \lambda-Q^{+}} A_{\zeta}(\lambda)_{\xi}
$$

We have a natural pairing

$$
\langle,\rangle: C_{\zeta} \times U_{\zeta}^{L} \rightarrow \mathbb{C}
$$

induced by (1.17). 
1.2.2. For a ring (resp., $\Lambda$-graded ring) $\mathcal{R}$ we denote by $\operatorname{Mod}(\mathcal{R})$ (resp., $\operatorname{Mod}_{\Lambda}(\mathcal{R})$ ) the category of $\mathcal{R}$-modules (resp., $\Lambda$-graded left $\mathcal{R}$-modules). Assume that we are given a homomorphism f $: A \rightarrow B$ of $\Lambda$-graded rings satisfying

$$
\jmath(A(\lambda)) B(\mu)=B(\mu) \jmath(A(\lambda)) \quad(\lambda, \mu \in \Lambda) .
$$

For $M \in \operatorname{Mod}_{\Lambda}(B)$, let $\operatorname{Tor}(M)$ be the subset of $M$ consisting of $m \in M$ such that there exists $\lambda \in \Lambda^{+}$satisfying $\jmath(A(\lambda+\mu)) m=\{0\}$ for any $\mu \in \Lambda^{+}$. Then $\operatorname{Tor}(M)$ is a subobject of $M$ in $\operatorname{Mod}_{\Lambda}(B)$ by (1.19). We denote by $\operatorname{Tor}_{\Lambda^{+}}(A, B)$ the full subcategory of $\operatorname{Mod}_{\Lambda}(B)$ consisting of $M \in \operatorname{Mod}_{\Lambda}(B)$ such that $\operatorname{Tor}(M)=M$. Note that $\operatorname{Tor}_{\Lambda^{+}}(A, B)$ is closed under taking subquotients and extensions in $\operatorname{Mod}_{\Lambda}(B)$. Let $\Sigma(A, B)$ denote the collection of morphisms $f$ of $\operatorname{Mod}_{\Lambda}(B)$ such that its kernel $\operatorname{Ker}(f)$ and its cokernel Coker $(f)$ belong to $\operatorname{Tor}_{\Lambda^{+}}(A, B)$. Then we define an abelian category $\mathcal{C}(A, B)=\operatorname{Mod}_{\Lambda}(B) / \operatorname{Tor}_{\Lambda^{+}}(A, B)$ as the localization

$$
\mathcal{C}(A, B)=\Sigma(A, B)^{-1} \operatorname{Mod}_{\Lambda}(B)
$$

of $\operatorname{Mod}_{\Lambda}(B)$ with respect to the multiplicative system $\Sigma(A, B)$ (see, e.g., [16] for the notion of localization of categories). We denote by

$$
\omega(A, B)^{*}: \operatorname{Mod}_{\Lambda}(B) \rightarrow \mathcal{C}(A, B)
$$

the canonical exact functor. It admits a right adjoint

$$
\omega(A, B)_{*}: \mathcal{C}(A, B) \rightarrow \operatorname{Mod}_{\Lambda}(B),
$$

which is left exact. It is known that $\omega(A, B)^{*} \circ \omega(A, B)_{*} \cong \mathrm{Id}$. By taking the degree 0 part of (1.21), we obtain a left exact functor

$$
\Gamma_{(A, B)}: \mathcal{C}(A, B) \rightarrow \operatorname{Mod}(B(0)) .
$$

The abelian category $\mathcal{C}(A, B)$ has enough injectives, and we have the right derived functors

$$
R^{i} \Gamma_{(A, B)}: \mathcal{C}(A, B) \rightarrow \operatorname{Mod}(B(0)) \quad(i \in \mathbb{Z})
$$

of $(1.22)$.

We apply the above arguments to the case $A=B=A_{\zeta}$. Then $\operatorname{Tor}(M)$ for $M \in \operatorname{Mod}_{\Lambda}\left(A_{\zeta}\right)$ consists of $m \in M$ such that there exists $\lambda \in \Lambda^{+}$satisfying $A_{\zeta}(\lambda) m=\{0\}$ (see [20, Lemma 3.4]). We set

$$
\operatorname{Mod}\left(\mathcal{O}_{\mathcal{B}_{\zeta}}\right)=\mathcal{C}\left(A_{\zeta}, A_{\zeta}\right)
$$


In this case, the natural functors $(1.20),(1.21),(1.22)$ are simply denoted as

$$
\begin{aligned}
& \omega^{*}: \operatorname{Mod} \Lambda\left(A_{\zeta}\right) \rightarrow \operatorname{Mod}\left(\mathcal{O}_{\mathcal{B}_{\zeta}}\right), \\
& \omega_{*}: \operatorname{Mod}\left(\mathcal{O}_{\mathcal{B}_{\zeta}}\right) \rightarrow \operatorname{Mod}_{\Lambda}\left(A_{\zeta}\right), \\
& \Gamma: \operatorname{Mod}\left(\mathcal{O}_{\mathcal{B}_{\zeta}}\right) \rightarrow \operatorname{Mod}(\mathbb{C}) .
\end{aligned}
$$

REMARK 1.5. In the terminology of noncommutative algebraic geometry, $\operatorname{Mod}\left(\mathcal{O}_{\mathcal{B}_{\zeta}}\right)$ is the category of quasicoherent sheaves on the quantized flag manifold $\mathcal{B}_{\zeta}$, which is a noncommutative projective scheme. The notations $\mathcal{B}_{\zeta}, \mathcal{O}_{\mathcal{B}_{\zeta}}$ have only symbolical meaning.

1.2.3. Using Lusztig's Frobenius homomorphism (1.12), we will relate the quantized flag manifold $\mathcal{B}_{\zeta}$ with the ordinary flag manifold $\mathcal{B}=B^{-} \backslash G$. Taking the dual Hopf algebras in (1.12), we obtain an injective homomorphism $\mathbb{C}[G] \rightarrow C_{\zeta}$ of Hopf algebras. Moreover, its image is contained in the center of $C_{\zeta}$ (see [14]). We will regard $\mathbb{C}[G]$ as a central Hopf subalgebra of $C_{\zeta}$ in the following. Setting

$$
\begin{aligned}
A_{1} & =\left\{\varphi \in \mathbb{C}[G] \mid \varphi(n g)=\varphi(g)\left(n \in N^{-}, g \in G\right)\right\}, \\
A_{1}(\lambda) & =\left\{\varphi \in A_{1} \mid \varphi(t g)=\theta_{\lambda}(t) \varphi(g)(t \in H, g \in G)\right\} \quad\left(\lambda \in \Lambda^{+}\right),
\end{aligned}
$$

we have a $\Lambda$-graded algebra

$$
A_{1}=\bigoplus_{\lambda \in \Lambda^{+}} A_{1}(\lambda) .
$$

We have a left $G$-module structure of $A_{1}$ given by

$$
(x \varphi)(g)=\varphi(g x) \quad\left(\varphi \in A_{1}, x, g \in G\right) .
$$

In particular, $A_{1}$ is a $U(\mathfrak{g})$-module. Moreover, for each $\lambda \in \Lambda^{+}, A_{1}(\lambda)$ is a $U(\mathfrak{g})$-submodule of $A_{1}$ which is an irreducible highest-weight module with highest-weight $\lambda$. Regarding $\mathbb{C}[G]$ as a subalgebra of $C_{\zeta}$, we have

$$
A_{1}=A_{\zeta} \cap \mathbb{C}[G], \quad A_{1}(\lambda)=A_{\zeta}(\ell \lambda) \cap \mathbb{C}[G] .
$$

Since the $\Lambda$-graded algebra $A_{1}$ is the homogeneous coordinate algebra of the projective variety $\mathcal{B}=B^{-} \backslash G$, we have an identification

$$
\operatorname{Mod}\left(\mathcal{O}_{\mathcal{B}}\right)=\mathcal{C}\left(A_{1}, A_{1}\right)
$$


of abelian categories, where $\operatorname{Mod}\left(\mathcal{O}_{\mathcal{B}}\right)$ denotes the category of quasicoherent $\mathcal{O}_{\mathcal{B}}$-modules on the ordinary flag manifold $\mathcal{B}$. We set

$$
\omega_{\mathcal{B} *}=\omega\left(A_{1}, A_{1}\right)_{*}: \operatorname{Mod}\left(\mathcal{O}_{\mathcal{B}}\right) \rightarrow \operatorname{Mod}_{\Lambda}\left(A_{1}\right) .
$$

For $\lambda \in \Lambda$, we denote by $\mathcal{O}_{\mathcal{B}}(\lambda) \in \operatorname{Mod}\left(\mathcal{O}_{\mathcal{B}}\right)$ the invertible $G$-equivariant $\mathcal{O}_{\mathcal{B}}$-module corresponding to $\lambda$. Then under identification (1.28), we have

$$
\omega_{\mathcal{B} *} M=\bigoplus_{\lambda \in \Lambda} \Gamma\left(\mathcal{B}, M \otimes_{\mathcal{O}_{\mathcal{B}}} \mathcal{O}_{\mathcal{B}}(\lambda)\right) \quad\left(M \in \operatorname{Mod}\left(\mathcal{O}_{\mathcal{B}}\right)\right),
$$

where $\Gamma(\mathcal{B}):, \operatorname{Mod}\left(\mathcal{O}_{\mathcal{B}}\right) \rightarrow \mathbb{C}$ is the global section functor for the algebraic variety $\mathcal{B}$. In particular, the functor $\Gamma_{\left(A_{1}, A_{1}\right)}: \operatorname{Mod}\left(\mathcal{O}_{\mathcal{B}}\right) \rightarrow \operatorname{Mod}(\mathbb{C})$ is identified with $\Gamma(\mathcal{B}$,$) .$

For a $\Lambda$-graded $\mathbb{C}$-algebra $B$, we define a new $\Lambda$-graded $\mathbb{C}$-algebra $B^{(\ell)}$ by

$$
B^{(\ell)}(\lambda)=B(\ell \lambda) \quad(\lambda \in \Lambda) .
$$

Let

$$
()^{(\ell)}: \operatorname{Mod}_{\Lambda}(B) \rightarrow \operatorname{Mod}_{\Lambda}\left(B^{(\ell)}\right)
$$

be the exact functor given by

$$
M^{(\ell)}(\lambda)=M(\ell \lambda) \quad(\lambda \in \Lambda)
$$

for $M \in \operatorname{Mod}_{\Lambda}(B)$.

We have the following results (see [20, Lemma 3.9]).

Lemma 1.6. Let $B$ be a $\Lambda$-graded $\mathbb{C}$-algebra. Assume that we are given a homomorphism $\mathrm{\jmath}: A_{\zeta} \rightarrow B$ of $\Lambda$-graded $\mathbb{C}$-algebras. We denote by $\jmath^{\prime}: A_{1} \rightarrow$ $B^{(\ell)}$ the induced homomorphism of $\Lambda$-graded $\mathbb{C}$-algebras. Assume that

$$
\begin{gathered}
\jmath\left(A_{\zeta}(\lambda)\right) B(\mu)=B(\mu) \jmath\left(A_{\zeta}(\lambda)\right) \quad(\lambda, \mu \in \Lambda), \\
\jmath^{\prime}\left(A_{1}(\lambda)\right) B^{(\ell)}(\mu)=B^{(\ell)}(\mu) \jmath^{\prime}\left(A_{1}(\lambda)\right) \quad(\lambda, \mu \in \Lambda) .
\end{gathered}
$$

Then the exact functor

$$
()^{(\ell)}: \operatorname{Mod}_{\Lambda}(B) \rightarrow \operatorname{Mod}_{\Lambda}\left(B^{(\ell)}\right)
$$

induces an equivalence

$$
\mathrm{Fr}_{*}: \mathcal{C}\left(A_{\zeta}, B\right) \rightarrow \mathcal{C}\left(A_{1}, B^{(\ell)}\right)
$$

of abelian categories. Moreover, we have

$$
\omega\left(A_{1}, B^{(\ell)}\right)_{*} \circ \operatorname{Fr}_{*}=()^{(\ell)} \circ \omega\left(A_{\zeta}, B\right)_{*} .
$$


Lemma 1.7. Let $F$ be a $\Lambda$-graded $\mathbb{C}$-algebra, and let $A_{1} \rightarrow F$ be a homomorphism of $\Lambda$-graded $\mathbb{C}$-algebras. Assume that $\operatorname{Im}\left(A_{1} \rightarrow F\right)$ is central in $F$. Regard $F$ as an object of $\operatorname{Mod}_{\Lambda}\left(A_{1}\right)$, and consider $\omega_{\mathcal{B}}^{*} F \in \operatorname{Mod}\left(\mathcal{O}_{\mathcal{B}}\right)$. Then the multiplication of $F$ induces an $\mathcal{O}_{\mathcal{B}}$-algebra structure of $\omega_{\mathcal{B}}^{*} F$, and we have an identification

$$
\mathcal{C}\left(A_{1}, F\right)=\operatorname{Mod}\left(\omega_{\mathcal{B}}^{*} F\right)
$$

of abelian categories, where $\operatorname{Mod}\left(\omega_{\mathcal{B}}^{*} F\right)$ denotes the category of quasicoherent $\omega_{\mathcal{B}}^{*} F$-modules. Moreover, under identification (1.33) we have

$$
\Gamma_{\left(A_{1}, F\right)}(M)=\Gamma(\mathcal{B}, M) \in \operatorname{Mod}(F(0)) \quad\left(M \in \operatorname{Mod}\left(\omega_{\mathcal{B}}^{*} F\right)\right)
$$

We define an $\mathcal{O}_{\mathcal{B}}$-algebra $\operatorname{Fr}_{*} \mathcal{O}_{\mathcal{B}_{\zeta}}$ by

$$
\operatorname{Fr}_{*} \mathcal{O}_{\mathcal{B}_{\zeta}}=\omega_{\mathcal{B}}^{*}\left(A_{\zeta}^{(\ell)}\right)
$$

We denote by $\operatorname{Mod}\left(\operatorname{Fr}_{*} \mathcal{O}_{\mathcal{B}_{\zeta}}\right)$ the category of quasicoherent $\operatorname{Fr}_{*} \mathcal{O}_{\mathcal{B}_{\zeta}}$-modules. By Lemmas 1.6 and 1.7, we have the following.

LEMMA 1.8. We have an equivalence

$$
\operatorname{Fr}_{*}: \operatorname{Mod}\left(\mathcal{O}_{\mathcal{B}_{\zeta}}\right) \rightarrow \operatorname{Mod}\left(\operatorname{Fr}_{*} \mathcal{O}_{\mathcal{B}_{\zeta}}\right)
$$

of abelian categories. Moreover, for $M \in \operatorname{Mod}\left(\mathcal{O}_{\mathcal{B}_{\zeta}}\right)$ we have

$$
R^{i} \Gamma(M) \simeq R^{i} \Gamma\left(\mathcal{B}, \operatorname{Fr}_{*}(M)\right)
$$

where $\Gamma(\mathcal{B}):, \operatorname{Mod}\left(\mathcal{O}_{\mathcal{B}}\right) \rightarrow \operatorname{Mod}(\mathbb{C})$ on the right-hand side is the global section functor for $\mathcal{B}$.

\section{$\S 2$. The category of $D$-modules}

\subsection{Ring of differential operators}

2.1.1. We define a subalgebra $D_{\mathbb{F}}$ of $\operatorname{End}_{\mathbb{F}}\left(A_{\mathbb{F}}\right)$ by

$$
D_{\mathbb{F}}=\left\langle\ell_{\varphi}, r_{\varphi}, \partial_{u}, \sigma_{\lambda} \mid \varphi \in A_{\mathbb{F}}, u \in U_{\mathbb{F}}, \lambda \in \Lambda\right\rangle,
$$

where

$$
\ell_{\varphi}(\psi)=\varphi \psi, \quad r_{\varphi}(\psi)=\psi \varphi, \quad \partial_{u}(\psi)=u \cdot \psi, \quad \sigma_{\lambda}(\psi)=q^{(\lambda, \mu)} \psi
$$


for $\psi \in A_{\mathbb{F}}(\mu)$. In fact, we have

$$
D_{\mathbb{F}}=\left\langle\ell_{\varphi}, \partial_{u}, \sigma_{\lambda} \mid \varphi \in A_{\mathbb{F}}, u \in U_{\mathbb{F}}, \lambda \in \Lambda\right\rangle
$$

by [20, Lemma 4.1].

We have a natural grading

$$
\begin{aligned}
D_{\mathbb{F}} & =\bigoplus_{\lambda \in \Lambda^{+}} D_{\mathbb{F}}(\lambda), \\
D_{\mathbb{F}}(\lambda) & =\left\{\Phi \in D_{\mathbb{F}} \mid \Phi\left(A_{\mathbb{F}}(\mu)\right) \subset A_{\mathbb{F}}(\lambda+\mu)(\mu \in \Lambda)\right\} \quad(\lambda \in \Lambda)
\end{aligned}
$$

of $D_{\mathbb{F}}$. It is easily checked that

$$
\begin{aligned}
& \partial_{u} \ell_{\varphi}=\sum_{(u)} \ell_{u_{(0)} \cdot \varphi} \partial_{u_{(1)}} \quad\left(u \in U_{\mathbb{F}}, \varphi \in A_{\mathbb{F}}\right), \\
& \partial_{u} \sigma_{\lambda}=\sigma_{\lambda} \partial_{u} \quad\left(u \in U_{\mathbb{F}}, \lambda \in \Lambda\right), \\
& \sigma_{\lambda} \ell_{\varphi}=q^{(\lambda, \mu)} \ell_{\varphi} \sigma_{\lambda} \quad\left(\lambda \in \Lambda, \varphi \in A_{\mathbb{F}}(\mu)\right) .
\end{aligned}
$$

Set

$$
E_{\mathbb{F}}=A_{\mathbb{F}} \otimes U_{\mathbb{F}} \otimes \mathbb{F}[\Lambda] .
$$

We have a natural $\mathbb{F}$-algebra structure of $E_{\mathbb{F}}$ such that $A_{\mathbb{F}} \otimes 1 \otimes 1,1 \otimes U_{\mathbb{F}} \otimes 1$, $1 \otimes 1 \otimes \mathbb{F}[\Lambda]$ are subalgebras of $E_{\mathbb{F}}$ naturally isomorphic to $A_{\mathbb{F}}, U_{\mathbb{F}}, \mathbb{F}[\Lambda]$, respectively, and such that we have the relations

$$
\begin{aligned}
u \varphi & =\sum_{(u)}\left(u_{(0)} \cdot \varphi\right) u_{(1)} \quad\left(u \in U_{\mathbb{F}}, \varphi \in A_{\mathbb{F}}\right), \\
u e(\lambda) & =e(\lambda) u \quad\left(u \in U_{\mathbb{F}}, \lambda \in \Lambda\right), \\
e(\lambda) \varphi & =q^{(\lambda, \mu)} \varphi e(\lambda) \quad\left(\lambda \in \Lambda, \varphi \in A_{\mathbb{F}}(\mu)\right)
\end{aligned}
$$

in $E_{\mathbb{F}}$. Here, we identify $A_{\mathbb{F}} \otimes 1 \otimes 1,1 \otimes U_{\mathbb{F}} \otimes 1,1 \otimes 1 \otimes \mathbb{F}[\Lambda]$ with $A_{\mathbb{F}}, U_{\mathbb{F}}$, $\mathbb{F}[\Lambda]$, respectively. Then we have a surjective algebra homomorphism

$$
E_{\mathbb{F}} \rightarrow D_{\mathbb{F}}
$$

sending $\varphi \in A_{\mathbb{F}}, u \in U_{\mathbb{F}}, e(\lambda) \in \mathbb{F}[\Lambda](\lambda \in \Lambda)$ to $\ell_{\varphi}, \partial_{u}, \sigma_{\lambda}$, respectively. Moreover, $E_{\mathbb{F}}$ has an obvious $\Lambda$-grading so that (2.1) preserves the $\Lambda$-grading. 
2.1.2. Set

$$
\begin{aligned}
D_{\mathbb{A}} & =\left\langle\ell_{\varphi}, r_{\varphi}, \partial_{u}, \sigma_{\lambda} \mid \varphi \in A_{\mathbb{A}}, u \in U_{\mathbb{A}}, \lambda \in \Lambda\right\rangle_{\mathbb{A}-\operatorname{alg}} \subset D_{\mathbb{F}}, \\
E_{\mathbb{A}} & =A_{\mathbb{A}} \otimes U_{\mathbb{A}} \otimes \mathbb{A}[\Lambda] \subset E_{\mathbb{A}} .
\end{aligned}
$$

They are $\Lambda$-graded $\mathbb{A}$-subalgebras of $D_{\mathbb{F}}$ and $E_{\mathbb{F}}$, respectively. Again, we have

$$
D_{\mathbb{A}}=\left\langle\ell_{\varphi}, \partial_{u}, \sigma_{\lambda} \mid \varphi \in A_{\mathbb{A}}, u \in U_{\mathbb{A}}, \lambda \in \Lambda\right\rangle_{\mathbb{A} \text {-alg }}
$$

by [20]. In particular, we have a surjective homomorphism

$$
E_{\mathbb{A}} \rightarrow D_{\mathbb{A}}
$$

of $\Lambda$-graded algebras. Note that there is a canonical embedding

$$
D_{\mathbb{A}} \rightarrow \operatorname{End}_{\mathbb{A}}\left(A_{\mathbb{A}}\right)
$$

2.1.3. We set

$$
D_{\zeta}=\mathbb{C} \otimes_{\mathbb{A}} D_{\mathbb{A}}, \quad E_{\zeta}=\mathbb{C} \otimes_{\mathbb{A}} E_{\mathbb{A}}=A_{\zeta} \otimes U_{\zeta} \otimes \mathbb{C}[\Lambda] .
$$

$D_{\zeta}$ is a $\Lambda$-graded $\mathbb{C}$-algebra generated by elements of the form

$$
\ell_{\varphi}, \quad \partial_{u}, \quad \sigma_{\lambda} \quad\left(\varphi \in A_{\zeta}, u \in U_{\zeta}, \lambda \in \Lambda\right)
$$

We have a surjective homomorphism

$$
E_{\zeta} \rightarrow D_{\zeta}
$$

of $\Lambda$-graded $\mathbb{C}$-algebras.

Lemma 2.1. Let $z \in Z_{\mathrm{Har}}\left(U_{\zeta}\right)$, and write $\iota(z)=\sum_{\lambda \in \Lambda} c_{\lambda} k_{2 \lambda}\left(c_{\lambda} \in \mathbb{C}\right)$. Then we have

$$
\partial_{z}=\sum_{\lambda \in \Lambda} c_{\lambda} \sigma_{2 \lambda} .
$$

Proof. This follows from the corresponding statement over $\mathbb{F}$, which is given in [19, Section 5.1].

REMARK 2.2. The natural algebra homomorphism $D_{\zeta} \rightarrow \operatorname{End}_{\mathbb{C}}\left(A_{\zeta}\right)$ is not injective. 
2.1.4. Define an $\mathcal{O}_{\mathcal{B}}$-algebra $\operatorname{Fr}_{*} \mathcal{D}_{\mathcal{B}_{\zeta}}$ by

$$
\operatorname{Fr}_{*} \mathcal{D}_{\mathcal{B}_{\zeta}}=\omega_{\mathcal{B}}^{*} D_{\zeta}^{(\ell)}
$$

We define $Z D_{\zeta}^{(\ell)}$ to be the central subalgebra of $D_{\zeta}^{(\ell)}$ generated by the elements of the form

$$
\ell_{\varphi}, \quad \partial_{u}, \quad \sigma_{\lambda} \quad\left(\varphi \in A_{1}, u \in Z_{\mathrm{Fr}}\left(U_{\zeta}\right), \lambda \in \Lambda\right)
$$

and we set

$$
\mathcal{Z}_{\zeta}=\omega_{\mathcal{B}}^{*} Z D_{\zeta}^{(\ell)}
$$

It is a central subalgebra of $\operatorname{Fr}_{*} \mathcal{D}_{\mathcal{B}_{\zeta}}$. Define a subvariety $\mathcal{V}$ of $\mathcal{B} \times K \times H$ by

$$
\mathcal{V}=\left\{\left(B^{-} g, k, t\right) \in \mathcal{B} \times K \times H \mid g \kappa(k) g^{-1} \in t^{2 \ell} N^{-}\right\}
$$

where $\kappa: K \rightarrow G$ is given by $\kappa\left(k_{1}, k_{2}\right)=k_{1} k_{2}^{-1}$. We denote by

$$
p_{\mathcal{V}}: \mathcal{V} \rightarrow \mathcal{B}
$$

the projection. Now we can state the main results of [20].

Theorem 2.3 ([20, Theorem 5.2]). The $\mathcal{O}_{\mathcal{B}}$-algebra $\mathcal{Z}_{\zeta}$ is naturally isomorphic to $p_{\mathcal{V} *} \mathcal{O}_{\mathcal{V}}$.

Define an $\mathcal{O}_{\mathcal{V}}$-algebra $\tilde{\mathcal{D}}_{\mathcal{B}_{\zeta}}$ by

$$
\tilde{\mathcal{D}}_{\mathcal{B}_{\zeta}}=p_{\mathcal{V}}^{-1} \operatorname{Fr}_{*} \mathcal{D}_{\mathcal{B}_{\zeta}} \otimes_{p_{\mathcal{V}}^{-1} p_{\mathcal{V} *} \mathcal{O}_{\mathcal{V}}} \mathcal{O}_{\mathcal{V}}
$$

TheOrem 2.4 ([20, Theorem 6.1]). Here $\tilde{\mathcal{D}}_{\mathcal{B}_{\zeta}}$ is an Azumaya algebra of $\operatorname{rank} \ell^{2\left|\Delta^{+}\right|}$.

Define

$$
\delta: \mathcal{V} \rightarrow K \times_{H / W} H
$$

by $\delta\left(B^{-} g, k, t\right)=(k, t)$, where $K \rightarrow H / W$ is given by $k \mapsto[h]$, where $h$ is an element of $H$ conjugate to the semisimple part of $\kappa(k)$, and $H \rightarrow H / W$ is given by $t \mapsto\left[t^{2 \ell}\right]$.

TheOREM 2.5 ([20, Theorem 6.10]). For any $(k, t) \in K \times_{H / W} H$, the restriction of $\tilde{\mathcal{D}}_{\mathcal{B}_{\zeta}}$ to $\delta^{-1}(k, t)$ is a split Azumaya algebra. 


\subsection{Category of $D$-modules}

We define an abelian category $\operatorname{Mod}\left(\mathcal{D}_{\mathcal{B}_{\zeta}}\right)$ by

$$
\operatorname{Mod}\left(\mathcal{D}_{\mathcal{B}_{\zeta}}\right)=\mathcal{C}\left(A_{\zeta}, D_{\zeta}\right)
$$

By Lemmas 1.6 and 1.7, we have an equivalence

$$
\operatorname{Fr}_{*}: \operatorname{Mod}\left(\mathcal{D}_{\mathcal{B}_{\zeta}}\right) \rightarrow \operatorname{Mod}\left(\operatorname{Fr}_{*} \mathcal{D}_{\mathcal{B}_{\zeta}}\right)
$$

of abelian categories, where $\operatorname{Mod}\left(\operatorname{Fr}_{*} \mathcal{D}_{\mathcal{B}_{\zeta}}\right)$ denotes the category of quasicoherent $\operatorname{Fr}_{*} \mathcal{D}_{\mathcal{B}_{\zeta}}$-modules. Moreover, for $M \in \operatorname{Mod}\left(\mathcal{D}_{\mathcal{B}_{\zeta}}\right)$ we have

$$
R^{i} \Gamma_{\left(A_{\zeta}, D_{\zeta}\right)}(M)=R^{i} \Gamma\left(\mathcal{B}, \operatorname{Fr}_{*}(M)\right) \in \operatorname{Mod}\left(D_{\zeta}(0)\right),
$$

where $\Gamma(\mathcal{B}$,$) on the right-hand side is the global section functor for the$ ordinary flag variety $\mathcal{B}$.

For $t \in H$ we define an abelian category $\operatorname{Mod}\left(\mathcal{D}_{\mathcal{B}_{\zeta}, t}\right)$ by

$$
\operatorname{Mod}\left(\mathcal{D}_{\mathcal{B}_{\zeta}, t}\right)=\operatorname{Mod}_{\Lambda, t}\left(D_{\zeta}\right) /\left(\operatorname{Mod}_{\Lambda, t}\left(D_{\zeta}\right) \cap \operatorname{Tor}_{\Lambda^{+}}\left(A_{\zeta}, D_{\zeta}\right)\right)
$$

where $\operatorname{Mod}_{\Lambda, t}\left(D_{\zeta}\right)$ is the full subcategory of $\operatorname{Mod}_{\Lambda}\left(D_{\zeta}\right)$ consisting of $M \in$ $\operatorname{Mod}_{\Lambda}\left(D_{\zeta}\right)$ so that $\left.\sigma_{\lambda}\right|_{M(\mu)}=\theta_{\lambda}(t) \zeta^{(\lambda, \mu)}$ id for any $\lambda, \mu \in \Lambda$. Then we can regard $\operatorname{Mod}\left(\mathcal{D}_{\mathcal{B}_{\zeta}, t}\right)$ as a full subcategory of $\operatorname{Mod}\left(\mathcal{D}_{\mathcal{B}_{\zeta}}\right)($ see [19, Lemma 4.6]). Set

$$
\operatorname{Fr}_{*} \mathcal{D}_{\mathcal{B}_{\zeta}, t}=\operatorname{Fr}_{*} \mathcal{D}_{\mathcal{B}_{\zeta}} \otimes_{\mathbb{C}[\Lambda]} \mathbb{C}_{t},
$$

where $\mathbb{C}_{t}$ denotes the 1-dimensional $\mathbb{C}[\Lambda]$-module given by $e(\lambda) \mapsto \theta_{\lambda}(t)$ for $\lambda \in \Lambda$. The equivalence (2.2) induces the equivalence

$$
\operatorname{Fr}_{*}: \operatorname{Mod}\left(\mathcal{D}_{\mathcal{B}_{\zeta}, t}\right) \rightarrow \operatorname{Mod}\left(\operatorname{Fr}_{*} \mathcal{D}_{\mathcal{B}_{\zeta}, t}\right)
$$

where $\operatorname{Mod}\left(\operatorname{Fr}_{*} \mathcal{D}_{\mathcal{B}_{\zeta}, t}\right)$ denotes the category of quasicoherent $\operatorname{Fr}_{*} \mathcal{D}_{\mathcal{B}_{\zeta}, t}$-modules. In particular, for $M \in \operatorname{Mod}\left(\mathcal{D}_{\mathcal{B}_{\zeta}, t}\right)$ we have

$$
R^{i} \Gamma_{\left(A_{\zeta}, D_{\zeta}\right)}(M)=R^{i} \Gamma\left(\mathcal{B}, \operatorname{Fr}_{*} M\right) \in \operatorname{Mod}\left(D_{\zeta, t}(0)\right),
$$

where $D_{\zeta, t}(0)=D_{\zeta}(0) / \sum_{\lambda \in \Lambda} D_{\zeta}(0)\left(\sigma_{\lambda}-\theta_{\lambda}(t)\right)$. 


\subsection{Conjecture}

By Lemma 2.1, the natural algebra homomorphism

$$
U_{\zeta} \otimes_{\mathbb{C}} \mathbb{C}[\Lambda] \rightarrow D_{\zeta}(0)
$$

factors through

$$
U_{\zeta} \otimes_{Z_{\mathrm{Har}}\left(U_{\zeta}\right)} \mathbb{C}[\Lambda] \rightarrow D_{\zeta}(0),
$$

where $Z_{\mathrm{Har}}\left(U_{\zeta}\right)$ is identified with $\mathbb{C}[2 \Lambda]^{W \circ}$ by (1.15). Hence, we have a natural algebra homomorphism

$$
U_{\zeta} \otimes_{Z_{\mathrm{Har}}\left(U_{\zeta}\right)} \mathbb{C}[\Lambda] \rightarrow \Gamma\left(\mathcal{B}, \operatorname{Fr}_{*} \mathcal{D}_{\mathcal{B}_{\zeta}}\right) .
$$

For $t \in H$ we denote by $\mathbb{C}_{t}$ the 1 -dimensional $\mathbb{C}[\Lambda]$-module given by $e(\lambda) v=$ $\theta_{\lambda}(t) v\left(v \in \mathbb{C}_{t}\right)$. Then (2.5) induces an algebra homomorphism

$$
U_{\zeta} \otimes_{Z_{\mathrm{Har}}\left(U_{\zeta}\right)} \mathbb{C}_{t} \rightarrow \Gamma\left(\mathcal{B}, \mathrm{Fr}_{*} \mathcal{D}_{\mathcal{B}_{\zeta}, t}\right),
$$

where $\mathbb{C}_{t}$ is regarded as a $Z_{\mathrm{Har}}\left(U_{\zeta}\right)$-module by $Z_{\mathrm{Har}}\left(U_{\zeta}\right) \cong \mathbb{C}[2 \Lambda]^{W \circ} \subset \mathbb{C}[\Lambda]$. Denote by $h_{G}$ the Coxeter number for $G$.

Conjecture 2.6. Assume that $\ell>h_{G}$. The algebra homomorphism (2.5) is an isomorphism, and we have

$$
R^{i} \Gamma\left(\mathcal{B}, \operatorname{Fr}_{*} \mathcal{D}_{\mathcal{B}_{\zeta}}\right)=0
$$

for $i \neq 0$.

Proposition 2.7. Let $\ell>h_{G}$, and assume that Conjecture 2.6 is valid. Then for $t \in H$ we have

$$
\Gamma\left(\mathcal{B}, \operatorname{Fr}_{*} \mathcal{D}_{\mathcal{B}_{\zeta}, t}\right) \cong U_{\zeta} \otimes_{Z_{\mathrm{Har}}\left(U_{\zeta}\right)} \mathbb{C}_{t}
$$

and

$$
R^{i} \Gamma\left(\mathcal{B}, \operatorname{Fr}_{*} \mathcal{D}_{\mathcal{B}_{\zeta}, t}\right)=0 \quad(i \neq 0)
$$

Proof. Define $f: \mathcal{V} \rightarrow H$ to be the composite of the embedding $\mathcal{V} \rightarrow \mathcal{B} \times$ $K \times H$ and the projection $\mathcal{B} \times K \times H \rightarrow H$ onto the third factor. Since $p_{\mathcal{V}}$ is an affine morphism, we have $R p_{\mathcal{V} *} \tilde{\mathcal{D}}_{\mathcal{B}_{\zeta}}=p_{\mathcal{V}_{*}} \tilde{\mathcal{D}}_{\mathcal{B}_{\zeta}}=\mathrm{Fr}_{*} \mathcal{D}_{\mathcal{B}_{\zeta}}$. Hence, we have

$$
U_{\zeta} \otimes_{Z_{\mathrm{Har}}\left(U_{\zeta}\right)}^{L} \mathbb{C}[\Lambda]=U_{\zeta} \otimes_{Z_{\mathrm{Har}}\left(U_{\zeta}\right)} \mathbb{C}[\Lambda] \cong R \Gamma\left(\mathcal{B}, \mathrm{Fr}_{*} \mathcal{D}_{\mathcal{B}_{\zeta}}\right)=R \Gamma\left(\mathcal{V}, \tilde{\mathcal{D}}_{\mathcal{B}_{\zeta}}\right)
$$


Here we use the fact that $\mathbb{C}[\Lambda]$ is a free $Z_{\text {Har }}\left(U_{\zeta}\right)$-module (see [17]). Denote by $\mathcal{O}_{t}$ the $\mathcal{O}_{H}$-module corresponding to the $\mathbb{C}[\Lambda]$-module $\mathbb{C}_{t}$. Similarly, we have

$$
\operatorname{Fr}_{*} \mathcal{D}_{\mathcal{B}_{\zeta}, t}=p_{\mathcal{V} *}\left(\tilde{\mathcal{D}}_{\mathcal{B}_{\zeta}} \otimes_{\mathbb{C}[\Lambda]} \mathbb{C}_{t}\right)=R p_{\mathcal{V} *}\left(\tilde{\mathcal{D}}_{\mathcal{B}_{\zeta}} \otimes_{\mathbb{C}[\Lambda]} \mathbb{C}_{t}\right)
$$

Since $f$ is flat, we have $L f^{*} \mathcal{O}_{t}=f^{*} \mathcal{O}_{t}$. Hence, by Theorem 2.4 we have

$$
\tilde{\mathcal{D}}_{\mathcal{B}_{\zeta}} \otimes_{\mathcal{O}_{\mathcal{V}}}^{L} L f^{*} \mathcal{O}_{t}=\tilde{\mathcal{D}}_{\mathcal{B}_{\zeta}} \otimes_{\mathcal{O}_{\mathcal{V}}}^{L} f^{*} \mathcal{O}_{t}=\tilde{\mathcal{D}}_{\mathcal{B}_{\zeta}} \otimes_{\mathcal{O}_{\mathcal{V}}} f^{*} \mathcal{O}_{t}
$$

It follows that

$$
\operatorname{Fr}_{*} \mathcal{D}_{\mathcal{B}_{\zeta}, t}=R p_{\mathcal{V}_{*}}\left(\tilde{\mathcal{D}}_{\mathcal{B}_{\zeta}} \otimes_{\mathcal{O}_{\mathcal{V}}}^{L} L f^{*} \mathcal{O}_{t}\right)=R p_{\mathcal{V}_{*}}\left(\tilde{\mathcal{D}}_{\mathcal{B}_{\zeta}}\right) \otimes_{\mathcal{O}_{H}}^{L} \mathcal{O}_{t}
$$

Hence we have

$$
\begin{aligned}
R \Gamma\left(\mathcal{B}, \operatorname{Fr}_{*} \mathcal{D}_{\mathcal{B}_{\zeta}, t}\right) & =R \Gamma\left(H, R f_{*}\left(\tilde{\mathcal{D}}_{\mathcal{B}_{\zeta}} \otimes_{\mathcal{O}_{\mathcal{V}}}^{L} L f^{*} \mathcal{O}_{t}\right)\right) \\
& =R \Gamma\left(H, R f_{*} \tilde{\mathcal{D}}_{\mathcal{B}_{\zeta}} \otimes_{\mathcal{O}_{H}}^{L} \mathcal{O}_{t}\right)=R \Gamma\left(H, R f_{*} \tilde{\mathcal{D}}_{\mathcal{B}_{\zeta}}\right) \otimes_{\mathbb{C}[\Lambda]}^{L} \mathbb{C}_{t} \\
& =R \Gamma\left(\mathcal{V}, \tilde{\mathcal{D}}_{\mathcal{B}_{\zeta}}\right) \otimes_{\mathbb{C}[\Lambda]}^{L} \mathbb{C}_{t}=U_{\zeta} \otimes_{Z_{\mathrm{Har}}\left(U_{\zeta}\right)}^{L} \mathbb{C}[\Lambda] \otimes_{\mathbb{C}[\Lambda]}^{L} \mathbb{C}_{t} \\
& =U_{\zeta} \otimes_{Z_{\mathrm{Har}}\left(U_{\zeta}\right)} \mathbb{C}_{t} .
\end{aligned}
$$

\subsection{Derived Beilinson-Bernstein equivalence}

We show that Conjecture 2.6 implies a variant of the Beilinson-Bernstein equivalence for derived categories.

Recall that we have an identification

$$
Z_{\mathrm{Har}}\left(U_{\zeta}\right) \cong \mathbb{C}[2 \Lambda]^{W \circ} \subset \mathbb{C}[2 \Lambda] \subset \mathbb{C}[\Lambda]
$$

Recall also that we identify $\mathbb{C}[\Lambda]$ with the coordinate algebra $\mathbb{C}[H]$ of $H$. Set $H^{(2)}=H / \operatorname{Ker}\left(H \ni t \mapsto t^{2} \in H\right)$, and let $\pi: H \rightarrow H^{(2)}$ be the canonical homomorphism. Then we have a natural identification $\mathbb{C}\left[H^{(2)}\right]=\mathbb{C}[2 \Lambda]$ so that $\pi^{*}: \mathbb{C}\left[H^{(2)}\right] \rightarrow \mathbb{C}[H]$ is identified with the inclusion $\mathbb{C}[2 \Lambda] \subset \mathbb{C}[\Lambda]$. Denote the isomorphism $H \cong H^{(2)}$ corresponding to $\mathbb{C}[\Lambda] \ni e(\lambda) \leftrightarrow e(2 \lambda) \in$ $\mathbb{C}[2 \Lambda]$ by $t \leftrightarrow t^{1 / 2}$. Then we have $\pi(t)=\left(t^{2}\right)^{1 / 2}$. The shifted action (1.13) of $W$ on $\mathbb{C}[2 \Lambda]$ induces an action of $W$ on $H^{(2)}$ given by

$$
w \circ t^{1 / 2}=\left(w\left(t t_{2 \rho}\right) t_{2 \rho}^{-1}\right)^{1 / 2} \quad(w \in W, t \in H),
$$


where $t_{2 \rho} \in H$ is given by $\theta_{\mu}\left(t_{2 \rho}\right)=\zeta^{2(\mu, \rho)}$ for any $\mu \in \Lambda$ (note that $2(\mu, \rho) \in$ $\mathbb{Z}$ ), and $Z_{\mathrm{Har}}\left(U_{\zeta}\right)$ is regarded as the coordinate algebra of the quotient variety $(W \circ) \backslash H^{(2)}$. For $t \in H$ we denote by $\chi_{t}: \mathbb{C}[\Lambda] \rightarrow \mathbb{C}$ the corresponding algebra homomorphism. By the above argument, we have

$$
\left.\chi_{t_{1}}\right|_{Z_{\mathrm{Har}}\left(U_{\zeta}\right)}=\left.\chi_{t_{2}}\right|_{Z_{\mathrm{Har}}\left(U_{\zeta}\right)} \Longleftrightarrow\left(t_{1}^{2}\right)^{1 / 2} \in W \circ t_{2}^{1 / 2} .
$$

We say that $t \in H$ is regular if

$$
\left\{w \in W \mid w \circ\left(t^{2}\right)^{1 / 2}=\left(t^{2}\right)^{1 / 2}\right\}=\{1\} .
$$

We denote by $\operatorname{Mod}_{\text {coh }}\left(\operatorname{Fr}_{*} \mathcal{D}_{\mathcal{B}_{\zeta}, t}\right)\left(\operatorname{resp} ., \operatorname{Mod}_{f}\left(U_{\zeta} \otimes_{Z_{\text {Har }}\left(U_{\zeta}\right)} \mathbb{C}_{t}\right)\right)$ the category of coherent $\operatorname{Fr}_{*} \mathcal{D}_{\mathcal{B}_{\zeta}, t}$-modules (resp., finitely generated $U_{\zeta} \otimes_{Z_{\mathrm{Har}}\left(U_{\zeta}\right)} \mathbb{C}_{t^{-}}$ modules). We also denote by $\operatorname{Mod}_{\text {coh }, t}\left(\operatorname{Fr}_{*} \mathcal{D}_{\mathcal{B}_{\zeta}}\right)\left(\operatorname{resp} ., \operatorname{Mod}_{f, t}\left(U_{\zeta}\right)\right)$ the category of coherent $\operatorname{Fr}_{*} \mathcal{D}_{\mathcal{B}_{\zeta}}$-modules (resp., finitely generated $U_{\zeta}$-modules) killed by some power of the maximal ideal of $\mathbb{C}[\Lambda]$ (resp., $Z_{\mathrm{Har}}\left(U_{\zeta}\right)$ ) corresponding to $t \in H$.

Theorem 2.8. Let $\ell>h_{G}$, and assume that Conjecture 2.6 is valid. If $t \in H$ is regular, then the natural functors

$$
\begin{aligned}
R \Gamma_{\hat{t}}: D^{b}\left(\operatorname{Mod}_{\mathrm{coh}, t}\left(\operatorname{Fr}_{*} \mathcal{D}_{\mathcal{B}_{\zeta}, t}\right)\right) & \rightarrow D^{b}\left(\operatorname{Mod}_{f, t}\left(U_{\zeta}\right)\right), \\
R \Gamma_{t}: D^{b}\left(\operatorname{Mod}_{\mathrm{coh}}\left(\operatorname{Fr}_{*} \mathcal{D}_{\mathcal{B}_{\zeta}, t}\right)\right) & \rightarrow D^{b}\left(\operatorname{Mod}_{f}\left(U_{\zeta} \otimes_{Z_{\mathrm{Har}}\left(U_{\zeta}\right)} \mathbb{C}_{t}\right)\right)
\end{aligned}
$$

give equivalences of derived categories.

The proof of this result is completely similar to that of the corresponding fact for Lie algebras in positive characteristics given in [6, Theorem 5.3.1]. We give below only an outline of it. First note the following.

Proposition 2.9 ([7, Theorem B]). Here $U_{\zeta}$ has finite homological dimension.

The functors

$$
\begin{aligned}
R \Gamma_{\hat{t}}: D^{b}\left(\operatorname{Mod}_{\mathrm{coh}, t}\left(\operatorname{Fr}_{*} \mathcal{D}_{\mathcal{B}_{\zeta}}\right)\right) & \rightarrow D^{b}\left(\operatorname{Mod}_{f, t}\left(U_{\zeta}\right)\right), \\
R \Gamma_{t}: D^{-}\left(\operatorname{Mod}_{\operatorname{coh}}\left(\operatorname{Fr}_{*} \mathcal{D}_{\mathcal{B}_{\zeta}, t}\right)\right) & \rightarrow D^{-}\left(\operatorname{Mod}_{f}\left(U_{\zeta} \otimes_{Z_{\mathrm{Har}}\left(U_{\zeta}\right)} \mathbb{C}_{t}\right)\right)
\end{aligned}
$$

have left adjoints

$$
\begin{aligned}
\mathcal{L}_{\hat{t}}: D^{b}\left(\operatorname{Mod}_{f, t}\left(U_{\zeta}\right)\right) & \rightarrow D^{b}\left(\operatorname{Mod}_{\mathrm{coh}, t}\left(\operatorname{Fr}_{*} \mathcal{D}_{\mathcal{B}_{\zeta}}\right)\right), \\
\mathcal{L}_{t}: D^{-}\left(\operatorname{Mod}_{f}\left(U_{\zeta} \otimes_{Z_{\mathrm{Har}}\left(U_{\zeta}\right)} \mathbb{C}_{t}\right)\right) & \rightarrow D^{-}\left(\operatorname{Mod}_{\operatorname{coh}}\left(\operatorname{Fr}_{*} \mathcal{D}_{\mathcal{B}_{\zeta}, t}\right)\right) .
\end{aligned}
$$

Arguing exactly as in [6, Sections 3.3, 3.4] using Theorem 2.4 and Proposition 2.9 , we obtain the following. 
Proposition 2.10.

(i) If $t$ is regular, the adjunction morphism $\mathrm{Id} \rightarrow R \Gamma_{\hat{t}} \circ \mathcal{L}_{\hat{t}}$ is an isomorphism on $D^{b}\left(\operatorname{Mod}_{f, t}\left(U_{\zeta}\right)\right)$.

(ii) For any $t$, the adjunction morphism $\mathrm{Id} \rightarrow R \Gamma_{t} \circ \mathcal{L}_{t}$ is an isomorphism on $D^{-}\left(\operatorname{Mod}_{f}\left(U_{\zeta} \otimes_{Z_{\mathrm{Har}}\left(U_{\zeta}\right)} \mathbb{C}_{t}\right)\right)$.

Arguing exactly as in [6, Section 3.5] using Theorem 2.4, Proposition 2.10, and Lemma 2.11 below, we obtain Theorem 2.8. Details are omitted.

LEMma 2.11 ([21, Section 2.4]). The variety $\mathcal{V}$ is a symplectic manifold.

\subsection{Finite part}

2.5.1. In [20, Section 4], we also introduced a quotient algebra $D_{\zeta}^{\prime}$ of $E_{\zeta}$, which is closely related to $D_{\zeta}$. Let us recall its definition. Take bases $\left\{x_{p}\right\}_{p}$, $\left\{y_{p}\right\}_{p},\left\{x_{p}^{L}\right\}_{p},\left\{y_{p}^{L}\right\}_{p}$ of $U_{\zeta}^{+}, U_{\zeta}^{-}, U_{\zeta}^{L,+}, U_{\zeta}^{L,-}$, respectively, such that

$$
\tau_{\zeta}^{L}\left(x_{p_{1}}, y_{p_{2}}^{L}\right)=\delta_{p_{1}, p_{2}}, \quad L_{\tau_{\zeta}}\left(x_{p_{1}}^{L}, y_{p_{2}}\right)=\delta_{p_{1}, p_{2}} .
$$

We assume that

$$
x_{p} \in U_{\zeta, \beta_{p}}^{+}, \quad y_{p} \in U_{\zeta,-\beta_{p}}^{-}, \quad x_{p}^{L} \in U_{\zeta, \beta_{p}}^{L,+}, \quad y_{p}^{L} \in U_{\zeta,-\beta_{p}}^{L,-}
$$

for $\beta_{p} \in Q^{+}$.

For $\varphi \in A_{\zeta}(\lambda)_{\xi}$ with $\lambda \in \Lambda^{+}, \xi \in \Lambda$, we set

$$
\begin{aligned}
& \Omega_{1}^{\prime}(\varphi)=\sum_{p}\left(y_{p}^{L} \cdot \varphi\right) x_{p} \in E_{\zeta, \diamond}, \\
& \Omega_{2}^{\prime}(\varphi)=\sum_{p}\left(\left(S x_{p}^{L}\right) \cdot \varphi\right) y_{p} k_{\beta_{p}} k_{2 \xi} e(-2 \lambda) \in E_{\zeta, \diamond}, \\
& \Omega^{\prime}(\varphi)=\Omega_{1}^{\prime}(\varphi)-\Omega_{2}^{\prime}(\varphi) \in E_{\zeta, \diamond} .
\end{aligned}
$$

We extend $\Omega^{\prime}$ to whole $A_{\zeta}$ by linearity. Then $D_{\zeta}^{\prime}$ is defined by

$$
D_{\zeta}^{\prime}=E_{\zeta} / \sum_{\varphi \in A_{\zeta}} A_{\zeta} \Omega^{\prime}(\varphi) U_{\zeta} \mathbb{C}[\Lambda]
$$

We have a sequence

$$
E_{\zeta} \rightarrow D_{\zeta}^{\prime} \rightarrow D_{\zeta}
$$

of surjective homomorphisms of $\Lambda$-graded algebras. Moreover, $D_{\zeta}^{\prime} \rightarrow D_{\zeta}$ induces an isomorphism

$$
\omega^{*} D_{\zeta}^{\prime} \cong \omega^{*} D_{\zeta}
$$

in $\operatorname{Mod}\left(\mathcal{O}_{\mathcal{B}_{\zeta}}\right)$ (see $[20$, Corollary 6.6]). 
2.5.2. We set

$$
U_{\mathbb{F}, \diamond}^{0}=\bigoplus_{\lambda \in \Lambda} \mathbb{F} k_{2 \lambda} \subset U_{\mathbb{F}}^{0}, \quad U_{\mathbb{F}, \diamond}=S\left(U_{\mathbb{F}}^{-}\right) U_{\mathbb{F}, \diamond}^{0} U_{\mathbb{F}}^{+} \subset U_{\mathbb{F}}
$$

Then we see easily the following.

LEMma 2.12. The subspace $U_{\mathbb{F}, \diamond}$ is an $\operatorname{ad}\left(U_{\mathbb{F}}\right)$-stable subalgebra of $U_{\mathbb{F}}$.

Set

$$
U_{\mathbb{F}, f}=\left\{u \in U_{\mathbb{F}} \mid \operatorname{dim} \operatorname{ad}\left(U_{\mathbb{F}}\right)(u)<\infty\right\} .
$$

Then $U_{\mathbb{F}, f}$ is a subalgebra of $U_{\mathbb{F}}$. Moreover, by [12] we have

$$
U_{\mathbb{F}, f}=\sum_{\lambda \in \Lambda^{+}} \operatorname{ad}\left(U_{\mathbb{F}}\right)\left(k_{-2 \lambda}\right),
$$

and hence $U_{\mathbb{F}, f}$ is a subalgebra of $U_{\mathbb{F}, \diamond}$. Note that $U_{\mathbb{F}, \diamond}$ and $U_{\mathbb{F}, f}$ are not Hopf subalgebras of $U_{\mathbb{F}}$; nevertheless, they satisfy the following.

LEMMA 2.13. We have

$$
\Delta\left(U_{\mathbb{F}, f}\right) \subset U_{\mathbb{F}} \otimes U_{\mathbb{F}, f}, \quad \Delta\left(U_{\mathbb{F}, \diamond}\right) \subset U_{\mathbb{F}} \otimes U_{\mathbb{F}, \diamond}
$$

Proof. For $u \in U_{\mathbb{F}}$ and $\lambda \in \Lambda^{+}$, we have

$$
\begin{aligned}
\Delta\left(\operatorname{ad}(u)\left(k_{-2 \lambda}\right)\right) & =\sum_{(u)} \Delta\left(u_{(0)} k_{-2 \lambda}\left(S u_{(1)}\right)\right) \\
& =\sum_{(u)_{3}} u_{(0)} k_{-2 \lambda}\left(S u_{(3)}\right) \otimes u_{(1)} k_{-2 \lambda}\left(S u_{(2)}\right) \\
& =\sum_{(u)_{2}} u_{(0)} k_{-2 \lambda}\left(S u_{(2)}\right) \otimes \operatorname{ad}\left(u_{(1)}\right)\left(k_{-2 \lambda}\right) .
\end{aligned}
$$

Hence, the first formula follows from (2.9). Since $U_{\mathbb{F}, \diamond}$ is generated by $e_{i}$, $S f_{i}$ for $i \in I$ and $k_{2 \lambda}$ for $\lambda \in \Lambda$, the second formula is a consequence of the fact that $\Delta\left(e_{i}\right), \Delta\left(S f_{i}\right), \Delta\left(k_{2 \lambda}\right)$ belong to $U_{\mathbb{F}} \otimes U_{\mathbb{F}, \diamond}$.

We set

$$
\begin{aligned}
& E_{\mathbb{F}, \diamond}=A_{\mathbb{F}} \otimes U_{\mathbb{F}, \diamond} \otimes \mathbb{F}[\Lambda] \subset E_{\mathbb{F}}, \\
& E_{\mathbb{F}, f}=A_{\mathbb{F}} \otimes U_{\mathbb{F}, f} \otimes \mathbb{F}[\Lambda] \subset E_{\mathbb{F}} .
\end{aligned}
$$


By Lemma 2.13, they are subalgebras of $E_{\mathbb{F}}$.

We set

$$
\begin{aligned}
& U_{\mathbb{A}, \diamond}^{0}=U_{\mathbb{F}, \diamond}^{0} \cap U_{\mathbb{A}}=\bigoplus_{\lambda \in \Lambda} \mathbb{A} k_{2 \lambda}, \quad U_{\mathbb{A}, \diamond}=U_{\mathbb{F}, \diamond} \cap U_{\mathbb{A}}=S\left(U_{\mathbb{A}}^{-}\right) U_{\mathbb{A}, \diamond}^{0} U_{\mathbb{A}}^{+}, \\
& U_{\mathbb{A}, f}=U_{\mathbb{A}} \cap U_{\mathbb{F}, f},
\end{aligned}
$$

and

$$
\begin{aligned}
& E_{\mathbb{A}, \diamond}=E_{\mathbb{A}} \cap E_{\mathbb{F}, \diamond}=A_{\mathbb{A}} \otimes U_{\mathbb{A}, \diamond} \otimes \mathbb{A}[\Lambda] \subset E_{\mathbb{F}, \diamond}, \\
& E_{\mathbb{A}, f}=E_{\mathbb{A}} \cap E_{\mathbb{F}, f}=A_{\mathbb{A}} \otimes U_{\mathbb{A}, f} \otimes \mathbb{A}[\Lambda] \subset E_{\mathbb{F}, f} .
\end{aligned}
$$

We also set

$$
\begin{aligned}
E_{\zeta, \diamond} & =\mathbb{C} \otimes_{\mathbb{A}} E_{\mathbb{A}, \diamond}=A_{\zeta} \otimes U_{\zeta, \diamond} \otimes \mathbb{C}[\Lambda] \subset E_{\zeta}, \\
E_{\zeta, f} & =\mathbb{C} \otimes_{\mathbb{A}} E_{\mathbb{A}, f}=A_{\zeta} \otimes U_{\zeta, f} \otimes \mathbb{C}[\Lambda] \subset E_{\zeta},
\end{aligned}
$$

and

$$
\begin{array}{ll}
D_{\zeta, \diamond}=\operatorname{Im}\left(E_{\zeta, \diamond} \rightarrow D_{\zeta}\right), & D_{\zeta, f}=\operatorname{Im}\left(E_{\zeta, f} \rightarrow D_{\zeta}\right), \\
D_{\zeta, \diamond}^{\prime}=\operatorname{Im}\left(E_{\zeta, \diamond} \rightarrow D_{\zeta}^{\prime}\right), & D_{\zeta, f}^{\prime}=\operatorname{Im}\left(E_{\zeta, f} \rightarrow D_{\zeta}^{\prime}\right) .
\end{array}
$$

By

$$
E_{\zeta} \cong E_{\zeta, \diamond} \otimes_{U_{\zeta, \diamond}} U_{\zeta}
$$

we obtain

$$
\begin{aligned}
& D_{\zeta, \diamond}^{\prime}=E_{\zeta, \diamond} / \sum_{\varphi \in A_{\zeta}} A_{\zeta} \Omega^{\prime}(\varphi) U_{\zeta, \diamond} \mathbb{C}[\Lambda], \\
& D_{\zeta}^{\prime} \cong D_{\zeta, \diamond}^{\prime} \otimes_{U_{\zeta, \diamond}} U_{\zeta}
\end{aligned}
$$

2.5.3. Since $U_{\zeta}$ is a free $U_{\zeta, \diamond}$-module, we have

$$
R^{i} \Gamma\left(\omega^{*} D_{\zeta}^{\prime}\right) \cong R^{i} \Gamma\left(\omega^{*} D_{\zeta, \diamond}^{\prime}\right) \otimes_{U_{\zeta, \diamond}} U_{\zeta}
$$

for any $i \in \mathbb{Z}$. Since $U_{\zeta, \diamond}$ is a localization of $U_{\zeta, f}$ with respect to the Ore subset $\left\{k_{-2 \lambda} \mid \Lambda \in \Lambda^{+}\right\}$, we have

$$
R^{i} \Gamma\left(\omega^{*} D_{\zeta, \diamond}^{\prime}\right) \cong R^{i} \Gamma\left(\omega^{*} D_{\zeta, f}^{\prime}\right) \otimes_{U_{\zeta, f}} U_{\zeta, \diamond}
$$


for any $i \in \mathbb{Z}$. It follows that

$$
R^{i} \Gamma\left(\omega^{*} D_{\zeta}^{\prime}\right) \cong R^{i} \Gamma\left(\omega^{*} D_{\zeta, f}^{\prime}\right) \otimes_{U_{\zeta, f}} U_{\zeta}
$$

for any $i \in \mathbb{Z}$. Note that

$$
R^{i} \Gamma\left(\mathcal{B}, \operatorname{Fr}_{*} \mathcal{D}_{\mathcal{B}_{\zeta}}\right) \cong R^{i} \Gamma\left(\omega^{*} D_{\zeta}^{\prime}\right)
$$

by Lemma 1.8 and (2.7). Hence Conjecture 2.6 is a consequence of the following stronger conjecture.

Conjecture 2.14. Assume that $\ell>h_{G}$. We have

$$
\Gamma\left(\omega^{*} D_{\zeta, f}^{\prime}\right) \cong U_{\zeta, f} \otimes_{Z_{\mathrm{Har}}\left(U_{\zeta}\right)} \mathbb{C}[\Lambda]
$$

and

$$
R^{i} \Gamma\left(\omega^{*} D_{\zeta, f}^{\prime}\right)=0
$$

for $i \neq 0$.

In the rest of this article, we give a reformulation of Conjecture 2.14 in terms of the induction functor.

\section{$\S 3 . \quad$ Representations}

\section{1 .}

For simplicity, we introduce a new notation, $\tilde{U}_{\mathbb{F}}^{-}=S\left(U_{\mathbb{F}}^{-}\right)$. Then we have $\tilde{U}_{\mathbb{F}}^{-}=\left\langle\tilde{f}_{i} \mid i \in I\right\rangle$, where $\tilde{f}_{i}=f_{i} k_{i}$ for $i \in I$. Moreover, setting

$$
\tilde{U}_{\mathbb{F}, \gamma}^{-}=\left\{u \in \tilde{U}_{\mathbb{F}}^{-} \mid k_{\mu} u k_{-\mu}=q^{(\gamma, \mu)} u(\mu \in \Lambda)\right\}
$$

for $\gamma \in Q$, we have

$$
\tilde{U}_{\mathbb{F}}^{-}=\bigoplus_{\gamma \in Q^{+}} \tilde{U}_{\mathbb{F},-\gamma}^{-}, \quad \tilde{U}_{\mathbb{F},-\gamma}^{-}=U_{\mathbb{F},-\gamma}^{-} k_{\gamma} \quad\left(\gamma \in Q^{+}\right) .
$$

We also set

$$
\begin{array}{lll}
\tilde{U}_{\mathbb{A}}=U_{\mathbb{A}} \cap \tilde{U}_{\mathbb{F}}, & \tilde{U}_{\mathbb{A},-\gamma}=U_{\mathbb{A}} \cap \tilde{U}_{\mathbb{F},-\gamma} & \left(\gamma \in Q^{+}\right), \\
\tilde{U}_{\zeta}=\mathbb{C} \otimes_{\mathbb{A}} \tilde{U}_{\mathbb{A}}, & \tilde{U}_{\zeta,-\gamma}=\mathbb{C} \otimes_{\mathbb{A}} \tilde{U}_{\mathbb{A},-\gamma} & \left(\gamma \in Q^{+}\right) .
\end{array}
$$

Then we have

$$
\tilde{U}_{\mathbb{A}}^{-}=\bigoplus_{\gamma \in Q^{+}} \tilde{U}_{\mathbb{A},-\gamma}^{-}, \quad \tilde{U}_{\zeta}^{-}=\bigoplus_{\gamma \in Q^{+}} \tilde{U}_{\zeta,-\gamma}^{-} .
$$




\section{2.}

For $\lambda \in \Lambda$, we define an algebra homomorphism $\chi_{\lambda}: U_{\mathbb{F}}^{0} \rightarrow \mathbb{F}$ by $\chi_{\lambda}\left(k_{\mu}\right)=$ $q^{(\lambda, \mu)}(\mu \in \Lambda)$. For $M \in \operatorname{Mod}\left(U_{\mathbb{F}}\right)$ and $\lambda \in \Lambda$, we set

$$
M_{\lambda}=\left\{m \in M \mid h m=\chi_{\lambda}(h) m\left(h \in U_{\mathbb{F}}^{0}\right)\right\} .
$$

For $\lambda \in \Lambda$, we define $M_{+, \mathbb{F}}(\lambda), M_{-, \mathbb{F}}(\lambda) \in \operatorname{Mod}\left(U_{\mathbb{F}}\right)$ by

$$
\begin{aligned}
& M_{+, \mathbb{F}}(\lambda)=U_{\mathbb{F}} / \sum_{y \in U_{\mathbb{F}}^{-}} U_{\mathbb{F}}(y-\varepsilon(y))+\sum_{h \in U_{\mathbb{F}}^{0}} U_{\mathbb{F}}\left(h-\chi_{\lambda}(h)\right), \\
& M_{-, \mathbb{F}}(\lambda)=U_{\mathbb{F}} / \sum_{x \in U_{\mathbb{F}}^{+}} U_{\mathbb{F}}(x-\varepsilon(x))+\sum_{h \in U_{\mathbb{F}}^{0}} U_{\mathbb{F}}\left(h-\chi_{\lambda}(h)\right),
\end{aligned}
$$

where $M_{+, \mathbb{F}}(\lambda)$ is a lowest-weight module with lowest-weight $\lambda$, and $M_{-, \mathbb{F}}(\lambda)$ is a highest-weight module with highest-weight $\lambda$. We have isomorphisms

$$
M_{+, \mathbb{F}}(\lambda) \cong U_{\mathbb{F}}^{+} \quad(\bar{u} \leftrightarrow u), \quad M_{-, \mathbb{F}}(\lambda) \cong U_{\mathbb{F}}^{-} \quad(\bar{u} \leftrightarrow u)
$$

of $\mathbb{F}$-modules. Moreover, we have weight-space decompositions

$$
M_{+, \mathbb{F}}(\lambda)=\bigoplus_{\mu \in \lambda+Q^{+}} M_{+, \mathbb{F}}(\lambda)_{\mu}, \quad M_{-, \mathbb{F}}(\lambda)=\bigoplus_{\mu \in \lambda-Q^{+}} M_{-, \mathbb{F}}(\lambda)_{\mu}
$$

For $\lambda \in \Lambda^{+}$we define $L_{+, \mathbb{F}}(-\lambda), L_{-, \mathbb{F}}(\lambda) \in \operatorname{Mod}_{f}\left(U_{\mathbb{F}}\right)$ by

$$
\begin{aligned}
L_{+, \mathbb{F}}(-\lambda)= & U_{\mathbb{F}} / \sum_{y \in U_{\mathbb{F}}^{-}} U_{\mathbb{F}}(y-\varepsilon(y)) \\
& +\sum_{h \in U_{\mathbb{F}}^{0}} U_{\mathbb{F}}\left(h-\chi_{-\lambda}(h)\right)+\sum_{i \in I} U_{\mathbb{F}} e_{i}^{\left(\left(\lambda, \alpha_{i}^{\vee}\right)+1\right)}, \\
L_{-, \mathbb{F}}(\lambda)= & U_{\mathbb{F}} / \sum_{x \in U_{\mathbb{F}}^{+}} U_{\mathbb{F}}(x-\varepsilon(x)) \\
& +\sum_{h \in U_{\mathbb{F}}^{0}} U_{\mathbb{F}}\left(h-\chi_{\lambda}(h)\right)+\sum_{i \in I} U_{\mathbb{F}} f_{i}^{\left(\left(\lambda, \alpha_{i}^{\vee}\right)+1\right)} .
\end{aligned}
$$

While $L_{+, \mathbb{F}}(-\lambda)$ is a finite-dimensional irreducible lowest-weight module with lowest-weight $-\lambda$, here $L_{-, \mathbb{F}}(\lambda)$ is a finite-dimensional irreducible 
highest-weight module with highest-weight $\lambda$. We have weight-space decompositions

$$
L_{+, \mathbb{F}}(-\lambda)=\bigoplus_{\mu \in-\lambda+Q^{+}} L_{+, \mathbb{F}}(-\lambda)_{\mu}, \quad L_{-, \mathbb{F}}(\lambda)=\bigoplus_{\mu \in \lambda-Q^{+}} L_{-, \mathbb{F}}(\lambda)_{\mu} .
$$

For $\lambda \in \Lambda^{+}$we have isomorphisms

$$
\begin{aligned}
L_{+, \mathbb{F}}(-\lambda) \cong U_{\mathbb{F}}^{L,+} / \sum_{i \in I} U_{\mathbb{F}}^{L,+} e_{i}^{\left(\left(\lambda, \alpha_{i}^{\vee}\right)+1\right)} & (\bar{u} \leftrightarrow \bar{u}), \\
L_{-, \mathbb{F}}(\lambda) \cong \tilde{U}_{\mathbb{F}}^{L,-} / \sum_{i \in I} \tilde{U}_{\mathbb{F}}^{L,-} \tilde{f}_{i}^{\left(\left(\lambda, \alpha_{i}^{\vee}\right)+1\right)} & (\bar{u} \leftrightarrow \bar{u})
\end{aligned}
$$

of vector spaces (see [13]).

Let $M$ be a $U_{\mathbb{F}^{-}}$-module with weight-space decomposition $M=\bigoplus_{\mu \in \Lambda} M_{\mu}$ such that $\operatorname{dim} M_{\mu}<\infty$ for any $\mu \in \Lambda$. We define a $U_{\mathbb{F}}$-module $M^{\star}$ by

$$
M^{\star}=\bigoplus_{\mu \in \Lambda} M_{\mu}^{*} \subset M^{*}=\operatorname{Hom}_{\mathbb{F}}(M, \mathbb{F}),
$$

where the action of $U_{\mathbb{F}}$ is given by

$$
\left\langle u m^{*}, m\right\rangle=\left\langle m^{*},(S u) m\right\rangle \quad\left(u \in U_{\mathbb{F}}, m^{*} \in M^{\star}, m \in M\right) .
$$

Here $\langle\rangle:, M^{\star} \times M \rightarrow \mathbb{F}$ is the natural pairing.

We set

$$
\begin{aligned}
M_{ \pm, \mathbb{F}}^{*}(\lambda) & =\left(M_{\mp, \mathbb{F}}(-\lambda)\right)^{\star} \quad(\lambda \in \Lambda), \\
L_{ \pm, \mathbb{F}}^{*}(\mp \lambda) & =\left(L_{\mp, \mathbb{F}}( \pm \lambda)\right)^{\star} \quad\left(\lambda \in \Lambda^{+}\right) .
\end{aligned}
$$

Since $L_{\mp, \mathbb{F}}( \pm \lambda)$ is irreducible, we have

$$
L_{ \pm, \mathbb{F}}^{*}(\mp \lambda) \cong L_{ \pm, \mathbb{F}}(\mp \lambda) \quad\left(\lambda \in \Lambda^{+}\right) .
$$

We define isomorphisms

$$
\Phi_{\lambda}: U_{\mathbb{F}}^{+} \rightarrow M_{+, \mathbb{F}}^{*}(\lambda), \quad \Psi_{\lambda}: \tilde{U}_{\mathbb{F}}^{-} \rightarrow M_{-, \mathbb{F}}^{*}(\lambda)
$$

of vector spaces by

$$
\begin{aligned}
\left\langle\Phi_{\lambda}(x), \bar{v}\right\rangle & =\tau(x, v) \quad\left(x \in U_{\mathbb{F}}^{+}, v \in \tilde{U}_{\mathbb{F}}^{-}\right), \\
\left\langle\Psi_{\lambda}(y), \overline{S u}\right\rangle & =\tau(u, y) \quad\left(y \in \tilde{U}_{\mathbb{F}}^{-}, u \in U_{\mathbb{F}}^{+}\right) .
\end{aligned}
$$


LEMMA 3.1.

(i) The $U_{\mathbb{F}}$-module structure of $M_{+, \mathbb{F}}^{*}(\lambda)$ is given by

$$
\begin{aligned}
& h \cdot \Phi_{\lambda}(x)=\chi_{\lambda+\gamma}(h) \Phi_{\lambda}(x) \quad\left(x \in U_{\mathbb{F}, \gamma}^{+}, h \in U_{\mathbb{F}}^{0}\right), \\
& v \cdot \Phi_{\lambda}(x)=\sum_{(x)} \tau\left(x_{(0)}, S v\right) \Phi_{\lambda}\left(x_{(1)}\right) \quad\left(x \in U_{\mathbb{F}}^{+}, v \in U_{\mathbb{F}}^{-}\right), \\
& u \cdot \Phi_{\lambda}(x)=\Phi_{\lambda}\left(k_{-\lambda}\left(\operatorname{ad}(u)\left(k_{\lambda} x k_{\lambda}\right)\right) k_{-\lambda}\right) \quad\left(x \in U_{\mathbb{F}}^{+}, u \in U_{\mathbb{F}}^{+}\right) .
\end{aligned}
$$

(ii) The $U_{\mathbb{F}}$-module structure of $M_{-, \mathbb{F}}^{*}(\lambda)$ is given by

$$
\begin{aligned}
& h \cdot \Psi_{\lambda}(y)=\chi_{\lambda-\gamma}(h) \Psi_{\lambda}(y) \quad\left(y \in \tilde{U}_{\mathbb{F},-\gamma}^{-}, h \in U_{\mathbb{F}}^{0}\right), \\
& u \cdot \Psi_{\lambda}(y)=\sum_{(y)} \tau\left(u, y_{(0)}\right) \Psi_{\lambda}\left(y_{(1)}\right) \quad\left(y \in \tilde{U}_{\mathbb{F}}^{-}, u \in U_{\mathbb{F}}^{+}\right), \\
& v \cdot \Psi_{\lambda}(y)=\Psi_{\lambda}\left(k_{\lambda}\left(\operatorname{ad}(v)\left(k_{-\lambda} y k_{-\lambda}\right)\right) k_{\lambda}\right) \quad\left(y \in \tilde{U}_{\mathbb{F}}^{-}, v \in U_{\mathbb{F}}^{-}\right) .
\end{aligned}
$$

Proof. We will prove only (i). The proof of (ii) is similar and omitted. Note that for $x \in U_{\mathbb{F}}^{+}, a \in U_{\mathbb{F}}$, and $v \in \tilde{U}_{\mathbb{F}}^{-}$, we have

$$
\left\langle a \cdot \Phi_{\lambda}(x), \bar{v}\right\rangle=\left\langle\Phi_{\lambda}(x), \overline{(S a) v}\right\rangle .
$$

Let us show (3.2). For $v \in \tilde{U}_{\mathbb{F},-\delta}^{-}$, we have

$$
\begin{aligned}
\left\langle h \cdot \Phi_{\lambda}(x), \bar{v}\right\rangle & =\left\langle\Phi_{\lambda}(x), \overline{(S h) v}\right\rangle=\delta_{\gamma, \delta}\left\langle\Phi_{\lambda}(x), \overline{(S h) v}\right\rangle \\
& =\delta_{\gamma, \delta} \chi_{\lambda+\gamma}(h)\left\langle\Phi_{\lambda}(x), \bar{v}\right\rangle=\chi_{\lambda+\gamma}(h)\left\langle\Phi_{\lambda}(x), \bar{v}\right\rangle .
\end{aligned}
$$

Hence, (3.2) holds. Let us next show (3.3). For $v \in \tilde{U}_{\mathbb{F}}^{-}$, we have

$$
\begin{aligned}
\left\langle y \cdot \Phi_{\lambda}(x), \bar{v}\right\rangle & =\left\langle\Phi_{\lambda}(x), \overline{(S y) v}\right\rangle=\tau(x,(S y) v)=\sum_{(x)} \tau\left(x_{(0)}, S y\right) \tau\left(x_{(1)}, v\right) \\
& =\left\langle\Phi_{\lambda}\left(\sum_{(x)} \tau\left(x_{(0)}, S y\right) x_{(1)}\right), \bar{v}\right\rangle .
\end{aligned}
$$

Hence, (3.3) also holds. Let us finally show (3.4). We may assume that $u \in U_{\mathbb{F}, \beta}^{+}$for some $\beta \in Q^{+}$. Then we can write

$$
\Delta u=\sum_{j} u_{j} k_{\beta_{j}^{\prime}} \otimes u_{j}^{\prime} \quad\left(\beta_{j}, \beta_{j}^{\prime} \in Q^{+}, \beta_{j}+\beta_{j}^{\prime}=\beta, u_{j} \in U_{\mathbb{F}, \beta_{j}}^{+}, u_{j}^{\prime} \in U_{\mathbb{F}, \beta_{j}^{\prime}}^{+}\right) .
$$


For $v \in \tilde{U}_{\mathbb{F}}^{-}$, we have

$$
\begin{aligned}
\left\langle u \cdot \Phi_{\lambda}(x), \bar{v}\right\rangle & =\left\langle\Phi_{\lambda}(x), \overline{(S u) v}\right\rangle \\
& =\sum_{(u)_{2},(v)_{2}} \tau\left(S u_{(2)}, v_{(0)}\right) \tau\left(S u_{(0)}, S v_{(2)}\right)\left\langle\Phi_{\lambda}(x), \overline{v_{(1)}\left(S u_{(1)}\right)}\right\rangle \\
& =\sum_{j,(v)_{2}} \tau\left(S u_{j}^{\prime}, v_{(0)}\right) \tau\left(u_{j} k_{\beta_{j}^{\prime}}, v_{(2)}\right)\left\langle\Phi_{\lambda}(x), \overline{v_{(1)}\left(S k_{\beta_{j}^{\prime}}\right)}\right\rangle \\
& =\sum_{j,(v)_{2}} q^{\left(\lambda, \beta_{j}^{\prime}-\beta_{j}\right)} \tau\left(S u_{j}^{\prime}, v_{(0)}\right) \tau\left(u_{j} k_{\beta_{j}^{\prime}}, v_{(2)}\right)\left\langle\Phi_{\lambda}(x), \overline{v_{(1)} k_{-\beta_{j}}}\right\rangle \\
& =\sum_{j,(v)_{2}} q^{\left(\lambda, \beta_{j}^{\prime}-\beta_{j}\right)} \tau\left(S u_{j}^{\prime}, v_{(0)}\right) \tau\left(u_{j} k_{\beta_{j}^{\prime}}, v_{(2)}\right) \tau\left(x, v_{(1)} k_{-\beta_{j}}\right) \\
& =\sum_{j,(v)_{2}} q^{\left(\lambda, \beta_{j}^{\prime}-\beta_{j}\right)} \tau\left(S u_{j}^{\prime}, v_{(0)}\right) \tau\left(x, v_{(1)}\right) \tau\left(u_{j} k_{\beta_{j}^{\prime}}, v_{(2)}\right) \\
= & \sum_{j} q^{\left(\lambda, \beta_{j}^{\prime}-\beta_{j}\right)} \tau\left(u_{j} k_{\beta_{j}^{\prime}} x\left(S u_{j}^{\prime}\right), v\right) \\
= & \left\langle\Phi_{\lambda}\left(k_{-\lambda}\left(\operatorname{ad}(u)\left(k_{\lambda} x k_{\lambda}\right)\right) k_{-\lambda}\right), \bar{v}\right\rangle .
\end{aligned}
$$

Here, we have used Lemma 1.1. Note also that $\Delta \tilde{U}_{\mathbb{F}}^{-} \subset \sum_{\gamma \in Q^{+}} \tilde{U}_{\mathbb{F}}^{-} k_{\gamma} \otimes$ $\tilde{U}_{\mathbb{F},-\gamma}^{-}$, and hence we have $\Delta_{2} \tilde{U}_{\mathbb{F}}^{-} \subset \sum_{\gamma, \delta \in Q^{+}} \tilde{U}_{\mathbb{F}}^{-} k_{\gamma+\delta} \otimes \tilde{U}_{\mathbb{F},-\gamma}^{-} k_{\delta} \otimes \tilde{U}_{\mathbb{F},-\delta}^{-}$. Thus, (3.4) is proved.

For $\lambda \in \Lambda$ we denote by $\mathbb{F}_{\lambda}^{\geq 0}=\mathbb{F} 1_{\lambda}^{\geqq 0}$ (resp., $\mathbb{F}_{\lambda}^{\leqq 0}=\mathbb{F} 1_{\lambda}^{\leqq 0}$ ) the 1-dimensional $U_{\mathbb{F}}^{\geqq 0}$-module(resp., $U_{\mathbb{F}}^{\leqq 0}$-module) such that $h 1_{\lambda}^{\geqq 0}=\chi_{\lambda}(h) 1_{\lambda}^{\geqq 0}, u 1_{\lambda}^{\geqq 0}=\varepsilon(u) 1_{\lambda}^{\geqq 0}$ for $h \in U_{\mathbb{F}}^{0}$ and $u \in U_{\mathbb{F}}^{+}$(resp., $h 1_{\bar{\lambda}}^{\leqq 0}=\chi_{\lambda}(h) 1_{\bar{\lambda}}^{\leqq 0}, u 1_{\bar{\lambda}}^{\leqq 0}=\varepsilon(u) 1_{\bar{\lambda}}^{\leqq 0}$ for $h \in U_{\mathbb{F}}^{0}$ and $\left.u \in U_{\mathbb{F}}^{-}\right)$.

Note that for any $\lambda \in \Lambda, k_{-2 \lambda} U_{\mathbb{F}}^{+}$(resp., $\tilde{U}_{\mathbb{F}}^{-} k_{-2 \lambda}$ ) is $\operatorname{ad}\left(U_{\mathbb{F}}^{\geqq 0}\right)$-stable (resp., $\operatorname{ad}\left(U_{\mathbb{F}}^{\leqq 0}\right)$-stable $)$. We see easily from Lemma 3.1 the following.

\section{LEMMA 3.2. Let $\lambda \in \Lambda$.}

(i) The linear map

$$
k_{-2 \lambda} U_{\mathbb{F}}^{+} \rightarrow M_{+, \mathbb{F}}^{*}(-\lambda) \otimes \mathbb{F}_{\lambda}^{\geqq 0} \quad\left(k_{-\lambda} x k_{-\lambda} \mapsto \Phi_{-\lambda}(x) \otimes 1_{\lambda}^{\geqq 0}\right)
$$

is an isomorphism of $U_{\mathbb{F}}^{\geqq 0}$-modules, where $k_{-2 \lambda} U_{\mathbb{F}}^{+}$is regarded as a $U_{\mathbb{F}}^{\leqq 0}$-module by the adjoint action. 
(ii) The linear map

$$
\tilde{U}_{\mathbb{F}}^{-} k_{-2 \lambda} \rightarrow \mathbb{F}_{-\lambda}^{\leqq 0} \otimes M_{-, \mathbb{F}}^{*}(\lambda) \quad\left(k_{-\lambda} y k_{-\lambda} \mapsto 1_{-\lambda}^{\leqq 0} \otimes \Psi_{\lambda}(y)\right)
$$

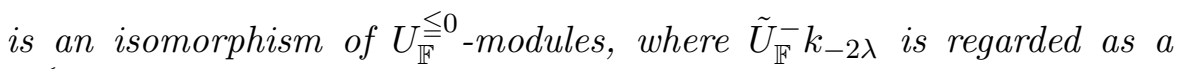
$U_{\mathbb{F}}^{\leq 0}$-module by the adjoint action.

We have an injective $U_{\mathbb{F}}$-homomorphism

$$
L_{ \pm, \mathbb{F}}^{*}(\mp \lambda) \rightarrow M_{ \pm, \mathbb{F}}^{*}(\mp \lambda) \quad\left(\lambda \in \Lambda^{+}\right)
$$

induced by the natural homomorphism $M_{ \pm, \mathbb{F}}(\mp \lambda) \rightarrow L_{ \pm, \mathbb{F}}(\mp \lambda)$. For $\lambda \in \Lambda^{+}$ we define subspaces $U_{\mathbb{F}}^{+}(\lambda), \tilde{U}_{\mathbb{F}}^{-}(\lambda)$ of $U_{\mathbb{F}}^{+}, \tilde{U}_{\mathbb{F}}^{-}$, respectively, by

$$
U_{\mathbb{F}}^{+}(\lambda)=\Phi_{-\lambda}^{-1}\left(L_{+, \mathbb{F}}^{*}(-\lambda)\right), \quad \tilde{U}_{\mathbb{F}}^{-}(\lambda)=\Psi_{\lambda}^{-1}\left(L_{-, \mathbb{F}}^{*}(\lambda)\right) .
$$

LEMMA 3.3.

(i) For $\lambda, \mu \in \Lambda^{+}$we have

$$
U_{\mathbb{F}}^{+}(\lambda) \subset U_{\mathbb{F}}^{+}(\lambda+\mu), \quad \tilde{U}_{\mathbb{F}}^{-}(\lambda) \subset \tilde{U}_{\mathbb{F}}^{-}(\lambda+\mu) .
$$

(ii) We have

$$
U_{\mathbb{F}}^{+}=\sum_{\lambda \in \Lambda^{+}} U_{\mathbb{F}}^{+}(\lambda), \quad \tilde{U}_{\mathbb{F}}^{-}=\sum_{\lambda \in \Lambda^{+}} \tilde{U}_{\mathbb{F}}^{-}(\lambda) .
$$

Proof. We will prove only the statements for $U_{\mathbb{F}}^{+}$. By definition, we have $U_{\mathbb{F}}^{+}(\lambda)=\left\{x \in U_{\mathbb{F}}^{+} \mid \tau\left(x, I_{\lambda}\right)=\{0\}\right\}$, where $I_{\lambda}=\sum_{i \in I} \tilde{U}_{\mathbb{F}}^{-} \tilde{f}_{i}^{\left(\left(\lambda, \alpha_{i}^{\vee}\right)+1\right)}$.

Hence, (i) is a consequence of $I_{\lambda} \supset I_{\lambda+\mu}$ for $\lambda, \mu \in \Lambda^{+}$. To show (ii) it is sufficient to show that for any $\beta \in Q^{+}$there exists some $\lambda \in \Lambda^{+}$such that $U_{\mathbb{F}, \beta}^{+} \subset U_{\mathbb{F}}^{+}(\lambda)$. Set $m=\operatorname{ht}(\beta)$. If $\lambda \in \Lambda^{+}$satisfies $\left(\lambda, \alpha_{i}^{\vee}\right) \geqq m$ for any $i \in I$, then we have $I_{\lambda} \subset \bigoplus_{\gamma \in Q^{+}, \operatorname{ht}(\gamma)>m} \tilde{U}_{\mathbb{F},-\gamma}^{-}$. From this we obtain $\tau\left(U_{\mathbb{F}, \beta}^{+}, I_{\lambda}\right)=$ $\{0\}$, and hence $U_{\mathbb{F}, \beta}^{+} \subset U_{\mathbb{F}}^{+}(\lambda)$.

Lemma 3.4. For $\lambda \in \Lambda^{+}$, we have

$$
\tilde{U}_{\mathbb{F}}^{-}(\lambda) k_{-2 \lambda} \subset U_{\mathbb{F}, f}, \quad k_{-2 \lambda} U_{\mathbb{F}}^{+}(\lambda) \subset U_{\mathbb{F}, f} .
$$

Proof. By Lemma 3.2, we have an isomorphism

$$
k_{-2 \lambda} U_{\mathbb{F}}^{+}(\lambda) \rightarrow L_{+, \mathbb{F}}^{*}(-\lambda) \otimes \mathbb{F}_{\bar{\lambda}}^{\geq 0} \quad\left(k_{-\lambda} x k_{-\lambda} \mapsto \Phi_{-\lambda}(x) \otimes 1_{\lambda}^{\geqq 0}\right)
$$


of $U_{\mathbb{F}}^{\geqq 0}$-modules. We have $L_{+, \mathbb{F}}^{*}(-\lambda) \cong L_{+, \mathbb{F}}(-\lambda)$, and hence $L_{+, \mathbb{F}}^{*}(-\lambda) \otimes \mathbb{F}_{\lambda}^{\geq 0}$ is generated by $\Phi_{-\lambda}(1) \otimes 1_{\bar{\lambda}}^{\geqq 0}$ as a $U_{\mathbb{F}}^{\geqq 0}$-module. It follows that

$$
k_{-2 \lambda} U_{\mathbb{F}}^{+}(\lambda)=\operatorname{ad}\left(U_{\mathbb{F}}^{\geqq 0}\right)\left(k_{-2 \lambda}\right) \subset U_{\mathbb{F}, f}
$$

by (2.9). The proof of $\tilde{U}_{\mathbb{F}}^{-}(\lambda) k_{-2 \lambda} \subset U_{\mathbb{F}, f}$ is similar.

\section{3.}

It is well known that, for $\lambda, \mu \in \Lambda$ such that $\lambda \neq \mu$, there exists $h \in U_{\mathbb{A}}^{L, 0}$ such that $\chi_{\lambda}(h)=1$ and $\chi_{\mu}(h)=0$. In particular, we have $\chi_{\lambda} \neq \chi_{\mu}$ (see, e.g., [20, Lemma 2.3]).

For $M \in \operatorname{Mod}\left(U_{\mathbb{A}}^{L}\right)$ and $\lambda \in \Lambda$, we set

$$
M_{\lambda}=\left\{m \in M \mid h m=\chi_{\lambda}(h) m\left(h \in U_{\mathbb{A}}^{L, 0}\right)\right\} .
$$

For $\lambda \in \Lambda$, we define $M_{+, \mathbb{A}}(\lambda), M_{-, \mathbb{A}}(\lambda) \in \operatorname{Mod}\left(U_{\mathbb{A}}^{L}\right)$ by

$$
\begin{aligned}
& M_{+, \mathbb{A}}(\lambda)=U_{\mathbb{A}}^{L} / \sum_{y \in U_{\mathbb{A}}^{L,-}} U_{\mathbb{A}}^{L}(y-\varepsilon(y))+\sum_{h \in U_{\mathbb{A}}^{L, 0}} U_{\mathbb{A}}^{L}\left(h-\chi_{\lambda}(h)\right), \\
& M_{-, \mathbb{A}}(\lambda)=U_{\mathbb{A}}^{L} / \sum_{x \in U_{\mathbb{A}}^{L,+}} U_{\mathbb{A}}^{L}(x-\varepsilon(x))+\sum_{h \in U_{\mathbb{A}}^{L, 0}} U_{\mathbb{A}}^{L}\left(h-\chi_{\lambda}(h)\right) .
\end{aligned}
$$

By the triangular decomposition we have isomorphisms

$$
M_{+, \mathbb{A}}(\lambda) \cong U_{\mathbb{A}}^{L,+} \quad(\bar{u} \leftrightarrow u), \quad M_{-, \mathbb{A}}(\lambda) \cong U_{\mathbb{A}}^{L,-} \quad(\bar{u} \leftrightarrow u)
$$

of $\mathbb{A}$-modules. In particular, $M_{ \pm, \mathbb{A}}(\lambda)$ is a free $\mathbb{A}$-module, and we have $\mathbb{F} \otimes_{\mathbb{A}}$ $M_{ \pm, \mathbb{A}}(\lambda) \cong M_{ \pm, \mathbb{F}}(\lambda)$. Moreover, we have weight-space decompositions

$$
M_{+, \mathbb{A}}(\lambda)=\bigoplus_{\mu \in \lambda+Q^{+}} M_{+, \mathbb{A}}(\lambda)_{\mu}, \quad M_{-, \mathbb{A}}(\lambda)=\bigoplus_{\mu \in \lambda-Q^{+}} M_{-, \mathbb{A}}(\lambda)_{\mu}
$$

For $\lambda \in \Lambda^{+}$, we define $L_{+, \mathbb{A}}(-\lambda) \in \operatorname{Mod}\left(U_{\mathbb{A}}^{L}\right)\left(\operatorname{resp} ., L_{-, \mathbb{A}}(\lambda) \in \operatorname{Mod}\left(U_{\mathbb{A}}^{L}\right)\right)$ to be the $U_{\mathbb{A}}^{L}$-submodule of $L_{+, \mathbb{F}}(-\lambda)$ (resp., $L_{-, \mathbb{F}}(\lambda)$ ) generated by $\overline{1} \in$ $L_{+, \mathbb{F}}(-\lambda)$ (resp., $\overline{1} \in L_{-, \mathbb{F}}(\lambda)$ ). By definition, $L_{ \pm, \mathbb{A}}(\mp \lambda)$ is a free $\mathbb{A}$-module, and we have $\mathbb{F} \otimes_{\mathbb{A}} L_{ \pm, \mathbb{A}}(\mp \lambda) \cong L_{ \pm, \mathbb{F}}(\mp \lambda)$. Moreover, we have weight-space decompositions

$$
L_{+, \mathbb{A}}(-\lambda)=\bigoplus_{\mu \in-\lambda+Q^{+}} L_{+, \mathbb{A}}(-\lambda)_{\mu}, \quad L_{-, \mathbb{A}}(\lambda)=\bigoplus_{\mu \in \lambda-Q^{+}} L_{-, \mathbb{A}}(\lambda)_{\mu}
$$


The canonical surjective $U_{\mathbb{F}}$-homomorphism $M_{ \pm, \mathbb{F}}(\mp \lambda) \rightarrow L_{ \pm, \mathbb{F}}(\mp \lambda)$ induces a surjective $U_{\mathbb{A}}^{L}$-homomorphism

$$
M_{ \pm, \mathbb{A}}(\mp \lambda) \rightarrow L_{ \pm, \mathbb{A}}(\mp \lambda) \quad\left(\lambda \in \Lambda^{+}\right) .
$$

Note that (3.9) is a split epimorphism of $\mathbb{A}$-modules since $\mathbb{A}$ is a PID (Principal Ideal Domain), and note that $M_{ \pm, \mathbb{A}}(\mp \lambda)_{\mu}, L_{ \pm, \mathbb{A}}(\mp \lambda)_{\mu}$ are torsion-free finitely generated $\mathbb{A}$-modules for each $\mu \in \Lambda$.

Let $M$ be a $U_{\mathbb{A}}^{L}$-module with weight-space decomposition $M=\bigoplus_{\mu \in \Lambda} M_{\mu}$ such that $M_{\mu}$ is a free $\mathbb{A}$-module of finite rank for any $\mu \in \Lambda$. We define a $U_{\mathbb{A}}^{L}$-module $M^{\star}$ by

$$
M^{\star}=\bigoplus_{\mu \in \Lambda} \operatorname{Hom}_{\mathbb{A}}\left(M_{\mu}, \mathbb{A}\right) \subset \operatorname{Hom}_{\mathbb{A}}(M, \mathbb{A}),
$$

where the action of $U_{\mathbb{A}}^{L}$ is given by

$$
\left\langle u m^{*}, m\right\rangle=\left\langle m^{*},(S u) m\right\rangle \quad\left(u \in U_{\mathbb{A}}^{L}, m^{*} \in M^{\star}, m \in M\right) .
$$

Here $\langle\rangle:, M^{\star} \times M \rightarrow \mathbb{A}$ is the natural pairing.

We set

$$
\begin{aligned}
M_{ \pm, \mathbb{A}}^{*}(\lambda) & =\left(M_{\mp, \mathbb{A}}(-\lambda)\right)^{\star} \quad(\lambda \in \Lambda), \\
L_{ \pm, \mathbb{A}}^{*}(\mp \lambda) & =\left(L_{\mp, \mathbb{A}}( \pm \lambda)\right)^{\star} \quad\left(\lambda \in \Lambda^{+}\right) .
\end{aligned}
$$

Then $M_{ \pm, \mathbb{A}}^{*}(\lambda)$ for $\lambda \in \Lambda$ and $L_{ \pm, \mathbb{A}}^{*}(\mp \lambda)$ for $\lambda \in \Lambda^{+}$are free $\mathbb{A}$-modules satisfying

$$
\mathbb{F} \otimes_{\mathbb{A}} M_{ \pm, \mathbb{A}}^{*}(\lambda) \cong M_{ \pm, \mathbb{F}}^{*}(\lambda), \quad \mathbb{F} \otimes_{\mathbb{A}} L_{ \pm, \mathbb{A}}^{*}(\mp \lambda) \cong L_{ \pm, \mathbb{F}}^{*}(\mp \lambda) .
$$

Moreover, we can identify $M_{ \pm, \mathbb{A}}^{*}(\lambda)$ and $L_{ \pm, \mathbb{A}}^{*}(\mp \lambda)$ with $\mathbb{A}$-submodules of $M_{ \pm, \mathbb{F}}^{*}(\lambda)$ and $L_{ \pm, \mathbb{F}}^{*}(\mp \lambda)$, respectively. Under this identification we have

$$
L_{ \pm, \mathbb{A}}^{*}(\mp \lambda)=L_{ \pm, \mathbb{F}}^{*}(\mp \lambda) \cap M_{ \pm, \mathbb{A}}^{*}(\mp \lambda) \quad\left(\lambda \in \Lambda^{+}\right) .
$$

In particular, the $U_{\mathbb{A}}^{L}$-homomorphism

$$
L_{ \pm, \mathbb{A}}^{*}(\mp \lambda) \rightarrow M_{ \pm, \mathbb{A}}^{*}(\mp \lambda) \quad\left(\lambda \in \Lambda^{+}\right)
$$

is a split monomorphism of $\mathbb{A}$-modules.

By abuse of notation we write

$$
\Phi_{\lambda}: U_{\mathbb{A}}^{+} \rightarrow M_{+, \mathbb{A}}^{*}(\lambda), \quad \Psi_{\lambda}: \tilde{U}_{\mathbb{A}}^{-} \rightarrow M_{-, \mathbb{A}}^{*}(\lambda)
$$

for the isomorphisms of $\mathbb{A}$-modules induced by (3.1). By Lemma 3.1 we have the following. 
LEMMA 3.5.

(i) The $U_{\mathbb{A}}^{L}$-module structure of $M_{+, \mathbb{A}}^{*}(\lambda)$ is given by

$$
\begin{aligned}
& h \cdot \Phi_{\lambda}(x)=\chi_{\lambda+\gamma}(h) \Phi_{\lambda}(x) \quad\left(x \in U_{\mathbb{A}, \gamma}^{+}, h \in U_{\mathbb{A}}^{L, 0}\right), \\
& v \cdot \Phi_{\lambda}(x)=\sum_{(x)} \tau_{\mathbb{A}}^{L}\left(x_{(0)}, S v\right) \Phi_{\lambda}\left(x_{(1)}\right) \quad\left(x \in U_{\mathbb{A}}^{+}, v \in U_{\mathbb{A}}^{L,-}\right), \\
& u \cdot \Phi_{\lambda}(x)=\Phi_{\lambda}\left(k_{-\lambda}\left(\operatorname{ad}(u)\left(k_{\lambda} x k_{\lambda}\right)\right) k_{-\lambda}\right) \quad\left(x \in U_{\mathbb{A}}^{+}, u \in U_{\mathbb{A}}^{L,+}\right) .
\end{aligned}
$$

(ii) The $U_{\mathbb{A}}^{L}$-module structure of $M_{-, \mathbb{A}}^{*}(\lambda)$ is given by

$$
\begin{aligned}
& h \cdot \Psi_{\lambda}(y)=\chi_{\lambda-\gamma}(h) \Psi_{\lambda}(y) \quad\left(y \in \tilde{U}_{\mathbb{A},-\gamma}^{-}, h \in U_{\mathbb{A}}^{L, 0}\right), \\
& u \cdot \Psi_{\lambda}(y)=\sum_{(y)}{ }^{L} \tau_{\mathbb{A}}\left(u, y_{(0)}\right) \Psi_{\lambda}\left(y_{(1)}\right) \quad\left(y \in \tilde{U}_{\mathbb{A}}^{-}, u \in U_{\mathbb{A}}^{L,+}\right), \\
& v \cdot \Psi_{\lambda}(y)=\Psi_{\lambda}\left(k_{\lambda}\left(\operatorname{ad}(v)\left(k_{-\lambda} y k_{-\lambda}\right)\right) k_{\lambda}\right) \quad\left(y \in \tilde{U}_{\mathbb{A}}^{-}, v \in U_{\mathbb{A}}^{L,-}\right) .
\end{aligned}
$$

For $\lambda \in \Lambda^{+}$we define $\mathbb{A}$-submodules $U_{\mathbb{A}}^{+}(\lambda), \tilde{U}_{\mathbb{A}}^{-}(\lambda)$ of $U_{\mathbb{A}}^{+}, \tilde{U}_{\mathbb{A}}^{-}$, respectively, by

$$
U_{\mathbb{A}}^{+}(\lambda)=\Phi_{-\lambda}^{-1}\left(L_{+, \mathbb{A}}^{*}(-\lambda)\right), \quad \tilde{U}_{\mathbb{A}}^{-}(\lambda)=\Psi_{\lambda}^{-1}\left(L_{-, \mathbb{A}}^{*}(\lambda)\right) .
$$

The embeddings

$$
U_{\mathbb{A}}^{+}(\lambda) \hookrightarrow U_{\mathbb{A}}^{+}, \quad \tilde{U}_{\mathbb{A}}^{-}(\lambda) \hookrightarrow \tilde{U}_{\mathbb{A}}^{-} \quad\left(\lambda \in \Lambda^{+}\right)
$$

are split monomorphisms of $\mathbb{A}$-modules. By (3.10), we have

$$
U_{\mathbb{A}}^{+}(\lambda)=U_{\mathbb{F}}^{+}(\lambda) \cap U_{\mathbb{A}}^{+}, \quad \tilde{U}_{\mathbb{A}}^{-}(\lambda)=\tilde{U}_{\mathbb{F}}^{-}(\lambda) \cap \tilde{U}_{\mathbb{A}}^{-} \quad\left(\lambda \in \Lambda^{+}\right) .
$$

In particular, we have

$$
\begin{aligned}
& U_{\mathbb{A}}^{+}(\lambda) \subset U_{\mathbb{A}}^{+}(\lambda+\mu), \quad \tilde{U}_{\mathbb{A}}^{-}(\lambda) \subset \tilde{U}_{\mathbb{A}}^{-}(\lambda+\mu) \quad\left(\lambda, \mu \in \Lambda^{+}\right), \\
& U_{\mathbb{A}}^{+}=\sum_{\lambda \in \Lambda^{+}} U_{\mathbb{A}}^{+}(\lambda), \quad \tilde{U}_{\mathbb{A}}^{-}=\sum_{\lambda \in \Lambda^{+}} \tilde{U}_{\mathbb{A}}^{-}(\lambda), \\
& \tilde{U}_{\mathbb{A}}^{-}(\lambda) k_{-2 \lambda} \subset U_{\mathbb{A}, f}, \quad k_{-2 \lambda} U_{\mathbb{A}}^{+}(\lambda) \subset U_{\mathbb{A}, f} \quad\left(\lambda \in \Lambda^{+}\right)
\end{aligned}
$$

by Lemmas 3.3 and 3.4 . 


\section{4 .}

Let $\lambda \in \Lambda$. By abuse of notation we also denote by $\chi_{\lambda}: U_{\zeta}^{L, 0} \rightarrow \mathbb{C}$ the $\mathbb{C}$-algebra homomorphism induced by $\chi_{\lambda}: U_{\mathbb{A}}^{L, 0} \rightarrow \mathbb{A}$. Then $\left\{\chi_{\lambda}\right\}_{\lambda \in \Lambda}$ is a linearly independent subset of the $\mathbb{C}$-module $\operatorname{Hom}_{\mathbb{C}}\left(U_{\zeta}^{L, 0}, \mathbb{C}\right)$. For $M \in \operatorname{Mod}\left(U_{\zeta}^{L}\right)$ and $\lambda \in \Lambda$, we set

$$
M_{\lambda}=\left\{m \in M \mid h m=\chi_{\lambda}(h) m\left(h \in U_{\zeta}^{L, 0}\right)\right\} .
$$

For $\lambda \in \Lambda$ we set

$$
M_{ \pm, \zeta}(\lambda)=\mathbb{C} \otimes_{\mathbb{A}} M_{ \pm, \mathbb{A}}(\lambda), \quad M_{ \pm, \zeta}^{*}(\lambda)=\mathbb{C} \otimes_{\mathbb{A}} M_{ \pm, \mathbb{A}}^{*}(\lambda)
$$

For $\lambda \in \Lambda^{+}$we set

$$
L_{ \pm, \zeta}(\mp \lambda)=\mathbb{C} \otimes_{\mathbb{A}} L_{ \pm, \mathbb{A}}(\mp \lambda), \quad L_{ \pm, \zeta}^{*}(\mp \lambda)=\mathbb{C} \otimes_{\mathbb{A}} L_{ \pm, \mathbb{A}}^{*}(\mp \lambda) .
$$

We have canonical $U_{\zeta}^{L}$-homomorphisms

$$
\begin{gathered}
M_{ \pm, \zeta}(\mp \lambda) \rightarrow L_{ \pm, \zeta}(\mp \lambda) \quad\left(\lambda \in \Lambda^{+}\right), \\
L_{ \pm, \zeta}^{*}(\mp \lambda) \rightarrow M_{ \pm, \zeta}^{*}(\mp \lambda) \quad\left(\lambda \in \Lambda^{+}\right) .
\end{gathered}
$$

Note that (3.24) is surjective and that (3.25) is injective.

For any $\lambda \in \Lambda^{+}$we have an isomorphism

$$
A_{\zeta}(\lambda) \cong L_{-, \zeta}^{*}(\lambda)
$$

of $U_{\zeta}^{L}$-modules (see, e.g., [11, Chapter 9], [20, Section 3.1]).

Let $\lambda \in \Lambda$. By abuse of notation we also denote by

$$
\Phi_{\lambda}: U_{\zeta}^{+} \rightarrow M_{+, \zeta}^{*}(\lambda), \quad \Psi_{\lambda}: \tilde{U}_{\zeta}^{-} \rightarrow M_{-, \zeta}^{*}(\lambda)
$$

the isomorphisms of $\mathbb{C}$-modules given by

$$
\begin{gathered}
\left\langle\Phi_{\lambda}(x), \bar{v}\right\rangle=\tau_{\zeta}^{L}(x, v) \quad\left(x \in U_{\zeta}^{+}, v \in \tilde{U}_{\zeta}^{L,-}\right), \\
\left\langle\Psi_{\lambda}(y), \overline{S u}\right\rangle={ }^{L} \tau_{\zeta}(u, y) \quad\left(y \in \tilde{U}_{\zeta}^{-}, u \in U_{\zeta}^{L,+}\right) .
\end{gathered}
$$

By Lemma 3.5, we have the following. 
LEMMA 3.6.

(i) The $U_{\zeta}^{L}$-module structure of $M_{+, \zeta}^{*}(\lambda)$ is given by

$$
\begin{aligned}
& h \cdot \Phi_{\lambda}(x)=\chi_{\lambda+\gamma}(h) \Phi_{\lambda}(x) \quad\left(x \in U_{\zeta, \gamma}^{+}, h \in U_{\zeta}^{L, 0}\right), \\
& v \cdot \Phi_{\lambda}(x)=\sum_{(x)} \tau_{\zeta}^{L}\left(x_{(0)}, S v\right) \Phi_{\lambda}\left(x_{(1)}\right) \quad\left(x \in U_{\zeta}^{+}, v \in U_{\zeta}^{L,-}\right), \\
& u \cdot \Phi_{\lambda}(x)=\Phi_{\lambda}\left(k_{-\lambda}\left(\operatorname{ad}(u)\left(k_{\lambda} x k_{\lambda}\right)\right) k_{-\lambda}\right) \quad\left(x \in U_{\zeta}^{+}, u \in U_{\zeta}^{L,+}\right) .
\end{aligned}
$$

(ii) The $U_{\zeta}^{L}$-module structure of $M_{-, \zeta}^{*}(\lambda)$ is given by

$$
\begin{aligned}
& h \cdot \Psi_{\lambda}(y)=\chi_{\lambda-\gamma}(h) \Psi_{\lambda}(y) \quad\left(y \in \tilde{U}_{\zeta,-\gamma}^{-}, h \in U_{\zeta}^{L, 0}\right), \\
& u \cdot \Psi_{\lambda}(y)=\sum_{(y)}{ }^{L} \tau_{\zeta}\left(u, y_{(0)}\right) \Psi_{\lambda}\left(y_{(1)}\right) \quad\left(y \in \tilde{U}_{\zeta}^{-}, u \in U_{\zeta}^{L,+}\right), \\
& v \cdot \Psi_{\lambda}(y)=\Psi_{\lambda}\left(k_{\lambda}\left(\operatorname{ad}(v)\left(k_{-\lambda} y k_{-\lambda}\right)\right) k_{\lambda}\right) \quad\left(y \in \tilde{U}_{\zeta}^{-}, v \in U_{\zeta}^{L,-}\right) .
\end{aligned}
$$

For $\lambda \in \Lambda^{+}$, we set

$$
\begin{aligned}
& U_{\zeta}^{+}(\lambda)=\mathbb{C} \otimes_{\mathbb{A}} U_{\mathbb{A}}^{+}(\lambda), \\
& \tilde{U}_{\zeta}^{-}(\lambda)=\mathbb{C} \otimes_{\mathbb{A}} \tilde{U}_{\mathbb{A}}^{-}(\lambda) .
\end{aligned}
$$

Then $U_{\zeta}^{+}(\lambda)$ and $\tilde{U}_{\zeta}^{-}(\lambda)$ are the $\mathbb{C}$-submodules of $U_{\zeta}^{+}$and $\tilde{U}_{\zeta}^{-}$, respectively, satisfying $\Phi_{-\lambda}\left(U_{\zeta}^{+}(\lambda)\right)=L_{+, \zeta}^{*}(-\lambda)$ and $\Psi_{\lambda}\left(\tilde{U}_{\zeta}^{-}(\lambda)\right)=L_{-, \zeta}^{*}(\lambda)$. We have linear isomorphisms

$$
\Phi_{-\lambda}: U_{\zeta}^{+}(\lambda) \rightarrow L_{+, \zeta}^{*}(-\lambda), \quad \Psi_{\lambda}: \tilde{U}_{\zeta}^{-}(\lambda) \rightarrow L_{-, \zeta}^{*}(\lambda) \quad\left(\lambda \in \Lambda^{+}\right)
$$

By (3.21), (3.22), and (3.23), we have

$$
\begin{aligned}
U_{\zeta}^{+}(\lambda) & \subset U_{\zeta}^{+}(\lambda+\mu), \quad \tilde{U}_{\zeta}^{-}(\lambda) \subset \tilde{U}_{\zeta}^{-}(\lambda+\mu) \quad\left(\lambda, \mu \in \Lambda^{+}\right), \\
U_{\zeta}^{+} & =\sum_{\lambda \in \Lambda^{+}} U_{\zeta}^{+}(\lambda), \quad \tilde{U}_{\zeta}^{-}=\sum_{\lambda \in \Lambda^{+}} \tilde{U}_{\zeta}^{-}(\lambda), \\
\tilde{U}_{\zeta}^{-}(\lambda) k_{-2 \lambda} & \subset U_{\zeta, f}, \quad k_{-2 \lambda} U_{\zeta}^{+}(\lambda) \subset U_{\mathbb{A}, f} \quad\left(\lambda \in \Lambda^{+}\right) .
\end{aligned}
$$

By (3.35) and (3.36), we can easily see the following. 
Lemma 3.7. For any $u \in U_{\zeta}$ there exists some $\lambda \in \Lambda^{+}$such that $u k_{-2 \lambda} \in$ $U_{\zeta, f}$.

\section{$\S 4$. Induction functor}

We set

$$
C_{\bar{\zeta}}^{\leqq 0}=C_{\zeta} / I, \quad I=\left\{\varphi \in C_{\zeta} \mid\left\langle\varphi, U_{\zeta}^{L, \leqq 0}\right\rangle=\{0\}\right\} .
$$

Then $C_{\bar{\zeta}}^{\leqq 0}$ is a Hopf algebra, and we have a Hopf pairing

$$
\langle,\rangle: C_{\zeta}^{\leqq 0} \times U_{\zeta}^{L, \leqq 0} \rightarrow \mathbb{C} .
$$

We have a canonical Hopf algebra homomorphism

$$
\text { res : } C_{\zeta} \rightarrow C_{\zeta}^{\leqq 0}
$$

Following Backelin and Kremnizer [2, Section 3], we define abelian categories $\mathcal{M}_{\zeta}$ and $\mathcal{M}_{\zeta}^{\mathrm{eq}}$ as follows.

An object of $\mathcal{M}_{\zeta}$ is a triplet $(M, \alpha, \beta)$ with

(1) $M$ a vector space over $\mathbb{C}$,

(2) $\alpha: C_{\zeta} \otimes M \rightarrow M$ a left $C_{\zeta}$-module structure of $M$,

(3) $\beta: M \rightarrow C_{\zeta}^{\leqq 0} \otimes M$ a left $C_{\zeta}^{\leqq 0}$-comodule structure of $M$

such that $\beta$ is a morphism of $C_{\zeta^{-}}$modules. (Or, equivalently, $\alpha$ is a morphism of $C_{\zeta}^{\leqq 0}$-comodules.) A morphism from $(M, \alpha, \beta)$ to $\left(M^{\prime}, \alpha^{\prime}, \beta^{\prime}\right)$ is a linear map $\varphi: M \rightarrow M^{\prime}$ which is a morphism of $C_{\zeta^{-}}$modules as well as that of $C_{\zeta}^{\leqq 0}$-comodules.

An object of $\mathcal{M}_{\zeta}^{\mathrm{eq}}$ is a quadruple $(M, \alpha, \beta, \gamma)$ with

(1) $M$ a vector space over $\mathbb{C}$,

(2) $\alpha: C_{\zeta} \otimes M \rightarrow M$ a left $C_{\zeta}$-module structure of $M$,

(3) $\beta: M \rightarrow C_{\bar{\zeta}}^{\leqq 0} \otimes M$ a left $C_{\bar{\zeta}}^{\leqq 0}$-comodule structure of $M$,

(4) $\gamma: M \rightarrow M \otimes C_{\zeta}$ a right $C_{\zeta}$-comodule structure of $M$

subject to the conditions that $(M, \alpha, \beta) \in \mathcal{M}_{\zeta}$, that $\beta$ and $\gamma$ commute with each other, and that $\gamma$ is a homomorphism of left $C_{\zeta^{-m o d u l e s}}$. A morphism from $(M, \alpha, \beta, \gamma)$ to $\left(M^{\prime}, \alpha^{\prime}, \beta^{\prime}, \gamma^{\prime}\right)$ is a linear map $\varphi: M \rightarrow M^{\prime}$ which is compatible with the left $C_{\zeta^{-}}$-module structure, the left $C_{\zeta}^{\leqq 0}$-comodule structure, and the right $C_{\zeta^{-}}$comodule structure. 
For a coalgebra $\mathcal{C}$ we denote by $\operatorname{Comod}(\mathcal{C})\left(\operatorname{resp} ., \operatorname{Comod}^{r}(\mathcal{C})\right)$ the category of left $\mathcal{C}$-comodules (resp., right $\mathcal{C}$-comodules). We define functors

$$
\begin{aligned}
\Xi: \mathcal{M}_{\zeta}^{\mathrm{eq}} & \rightarrow \operatorname{Comod}\left(C_{\zeta}^{\leqq 0}\right), \\
\Upsilon: \operatorname{Comod}\left(C_{\zeta}^{\leqq 0}\right) & \rightarrow \mathcal{M}_{\zeta}^{\mathrm{eq}}
\end{aligned}
$$

by

$$
\begin{aligned}
& \Xi(M)=\{M \in M \mid \gamma(m)=m \otimes 1\}, \\
& \Upsilon(L)=C_{\zeta} \otimes L .
\end{aligned}
$$

By Backelin and Kremnizer [2, Section 3.5], we have the following.

Proposition 4.1. The functor $\Xi: \mathcal{M}_{\zeta}^{\mathrm{eq}} \rightarrow \operatorname{Comod}\left(C_{\zeta}^{\leqq 0}\right)$ gives an equivalence of categories, and its quasi-inverse is given by $\Upsilon$.

REMARK 4.2. For $M \in \mathcal{M}_{\zeta}^{\mathrm{eq}}$ we have an isomorphism

$$
\Xi(M) \cong \mathbb{C} \otimes C_{\zeta} M
$$

of vector spaces by Proposition 4.1. Here $C_{\zeta} \rightarrow \mathbb{C}$ is given by $\varepsilon$.

For $\lambda \in \Lambda$ we define $\chi_{\lambda}^{\leqq 0} \in C_{\zeta}^{\leqq 0} \subset \operatorname{Hom}_{\mathbb{C}}\left(U_{\zeta}^{L, \leqq 0}, \mathbb{C}\right)$ by

$$
\chi_{\bar{\lambda}}^{\leqq 0}(h u)=\chi_{\lambda}(h) \varepsilon(u) \quad\left(h \in U_{\zeta}^{L, 0}, u \in U_{\zeta}^{L,-}\right) .
$$

We define left exact functors

$$
\begin{gathered}
\omega_{\mathcal{M} *}: \mathcal{M}_{\zeta} \rightarrow \operatorname{Mod}_{\Lambda}\left(A_{\zeta}\right), \\
\Gamma_{\mathcal{M}}: \mathcal{M}_{\zeta} \rightarrow \operatorname{Mod}(\mathbb{C})
\end{gathered}
$$

by

$$
\begin{aligned}
\omega_{\mathcal{M} *}(M) & =\bigoplus_{\lambda \in \Lambda}\left(\omega_{\mathcal{M} *}(M)\right)(\lambda) \subset M, \\
\left(\omega_{\mathcal{M} *}(M)\right)(\lambda) & =\left\{m \in M \mid \beta(m)=\chi_{\lambda}^{\leqq 0} \otimes m\right\}, \\
\Gamma_{\mathcal{M}}(M) & =\left(\omega_{\mathcal{M} *}(M)\right)(0) .
\end{aligned}
$$

We denote by $\operatorname{Mod}_{\Lambda}^{\mathrm{eq}}\left(A_{\zeta}\right)$ the category consisting of $N \in \operatorname{Mod}_{\Lambda}\left(A_{\zeta}\right)$ equipped with a right $C_{\zeta}$-comodule structure $\gamma: N \rightarrow N \otimes C_{\zeta}$ such that 
$\gamma(N(\lambda)) \subset N(\lambda) \otimes C_{\zeta}$ for any $\lambda \in \Lambda$ and $\gamma(\varphi n)=\Delta(\varphi) \gamma(n)$ for any $\varphi \in A_{\zeta}$ and $n \in N$. (Note that $\Delta\left(A_{\zeta}(\lambda)\right) \subset A_{\zeta}(\lambda) \otimes C_{\zeta}$.) By definition, (4.1) and (4.2) induce left exact functors

$$
\begin{gathered}
\omega_{\mathcal{M} *}^{\mathrm{eq}}: \mathcal{M}_{\zeta}^{\mathrm{eq}} \rightarrow \operatorname{Mod}_{\Lambda}^{\mathrm{eq}}\left(A_{\zeta}\right), \\
\Gamma_{\mathcal{M}}^{\mathrm{eq}}: \mathcal{M}_{\zeta}^{\mathrm{eq}} \rightarrow \operatorname{Comod}^{r}\left(C_{\zeta}\right)
\end{gathered}
$$

We also define a left exact functor

$$
\text { Ind }: \operatorname{Comod}\left(C_{\zeta}^{\leqq 0}\right) \rightarrow \operatorname{Comod}^{r}\left(C_{\zeta}\right)
$$

by Ind $=\Gamma_{\mathcal{M}}^{\mathrm{eq}} \circ \Upsilon$.

The abelian categories $\mathcal{M}_{\zeta}, \mathcal{M}_{\zeta}^{\text {eq }}, \operatorname{Comod}^{r}\left(C_{\zeta}\right)$ have enough injectives, and the forgetful functor $\mathcal{M}_{\zeta}^{\mathrm{eq}} \rightarrow \mathcal{M}_{\zeta}$ sends injective objects to $\Gamma_{\mathcal{M}}$-acyclic objects (see [2, Section 3.4]). Hence, we have the following.

LEMMA 4.3. We have

$$
\begin{gathered}
\text { For } \circ R^{i} \Gamma_{\mathcal{M}}^{\mathrm{eq}}=R^{i} \Gamma_{\mathcal{M}} \circ \text { For }: \mathcal{M}_{\zeta}^{\mathrm{eq}} \rightarrow \operatorname{Mod}(\mathbb{C}) \\
R^{i} \text { Ind } \circ \Xi=R^{i} \Gamma_{\mathcal{M}}^{\mathrm{eq}}: \mathcal{M}_{\zeta}^{\mathrm{eq}} \rightarrow \operatorname{Comod}^{r}\left(C_{\zeta}\right)
\end{gathered}
$$

for any $i$, where For $: \operatorname{Comod}^{r}\left(C_{\zeta}\right) \rightarrow \operatorname{Mod}(\mathbb{C})$ and For $: \mathcal{M}_{\zeta}^{\mathrm{eq}} \rightarrow \mathcal{M}_{\zeta}$ are forgetful functors.

We define an exact functor

$$
\text { res : } \operatorname{Comod}^{r}\left(C_{\zeta}\right) \rightarrow \operatorname{Comod}\left(C_{\bar{\zeta}}^{\leqq 0}\right)
$$

as follows. For $V \in \operatorname{Comod}^{r}\left(C_{\zeta}\right)$ with right $C_{\zeta}$-comodule structure $\beta: V \rightarrow$ $V \otimes C_{\zeta}$, we have $\operatorname{res}(V)=V$ as a $\mathbb{C}$-module, and the left $C_{\bar{\zeta}}^{\leqq 0}$-comodule structure $\operatorname{res}(V) \rightarrow C_{\bar{\zeta}}^{\leqq 0} \otimes \operatorname{res}(V)$ of $\operatorname{res}(V)$ is given by

$$
\beta(v)=\sum_{k} v_{k} \otimes \varphi_{k} \quad \Longrightarrow \quad \gamma(v)=\sum_{k} \operatorname{res}\left(S^{-1} \varphi_{k}\right) \otimes v_{k}
$$

The following fact is standard.

Lemma 4.4. For $V \in \operatorname{Comod}^{r}\left(C_{\zeta}\right), M \in \operatorname{Comod}\left(C_{\zeta}^{\leqq 0}\right)$, we have an isomorphism

$$
F: \operatorname{Ind}(M) \otimes V \rightarrow \operatorname{Ind}(\operatorname{res}(V) \otimes M)
$$


of right $C_{\zeta}$-comodules given by

$$
F\left(\left(\sum_{i} \varphi_{i} \otimes m_{i}\right) \otimes v\right)=\sum_{i,(v)} \varphi_{i} v_{(1)} \otimes v_{(0)} \otimes m_{i}
$$

where we write the right $C_{\zeta}$-comodule structure of $V$ by

$$
V \ni v \mapsto \sum_{(v)} v_{(0)} \otimes v_{(1)} \in V \otimes C_{\zeta}
$$

For $\lambda \in \Lambda$ we denote by $\mathbb{C}_{\lambda}^{\leq 0}=\mathbb{C} 1_{\lambda}^{\leq 0}$ the object of $\operatorname{Comod}\left(C_{\bar{\zeta}}^{\leq 0}\right)$ corresponding to the 1 -dimensional right $U_{\zeta}^{L, \leqq 0}$-module given by $1_{\lambda}^{\leqq 0} u=\chi_{\lambda}^{\leqq 0}(u) 1_{\lambda}^{\leqq 0}$ for $u \in U_{\zeta}^{L, \leqq 0}$. By definition, we have an isomorphism

$$
\operatorname{Ind}\left(\mathbb{C}_{-\lambda}^{\leqq 0}\right) \cong A_{\zeta}(\lambda) \quad\left(\lambda \in \Lambda^{+}\right)
$$

of right $C_{\zeta^{-}}$comodules.

Let $N \in \operatorname{Mod}_{\Lambda}\left(A_{\zeta}\right)$. Then $C_{\zeta} \otimes_{A_{\zeta}} N$ turns out to be an object of $\mathcal{M}_{\zeta}$ by

$$
\begin{aligned}
\alpha\left(f \otimes\left(f^{\prime} \otimes n\right)\right) & =f f^{\prime} \otimes n \quad\left(f, f^{\prime} \in C_{\zeta}, n \in N\right), \\
\beta(f \otimes n) & =\sum_{(f)} \operatorname{res}\left(f_{(0)}\right) \chi_{\lambda} \otimes\left(f_{(1)} \otimes n\right) \quad\left(f \in C_{\zeta}, n \in N(\lambda)\right) .
\end{aligned}
$$

Hence, we have a functor $\operatorname{Mod}_{\Lambda}\left(A_{\zeta}\right) \rightarrow \mathcal{M}_{\zeta}$ sending $N$ to $C_{\zeta} \otimes_{A_{\zeta}} N$.

Lemma 4.5. The functor $\operatorname{Mod}_{\Lambda}\left(A_{\zeta}\right) \rightarrow \mathcal{M}_{\zeta}$ as above induces a functor

$$
\Phi: \operatorname{Mod}\left(\mathcal{O}_{\mathcal{B}_{\zeta}}\right) \rightarrow \mathcal{M}_{\zeta}
$$

Proof. It is sufficient to show that $C_{\zeta} \otimes_{A_{\zeta}} A_{\zeta} / A_{\zeta}\left(\lambda+\Lambda^{+}\right)=\{0\}$ for any $\lambda \in \Lambda$. Hence, we have only to show that $C_{\zeta} A_{\zeta}(\lambda)=C_{\zeta}$ for any $\lambda \in \Lambda^{+}$. Take $\varphi \in A_{\zeta}(\lambda)$ such that $\varepsilon(\varphi)=1$. We have $\Delta\left(A_{\zeta}(\lambda)\right) \subset A_{\zeta}(\lambda) \otimes C_{\zeta}$, and hence we can write $\Delta(\varphi)=\sum_{i} \varphi_{i} \otimes \varphi_{i}^{\prime}$ with $\varphi_{i} \in A_{\zeta}(\lambda), \varphi_{i}^{\prime} \in C_{\zeta}$. Then we have $C_{\zeta} A_{\zeta}(\lambda) \ni \sum_{i}\left(S^{-1} \varphi_{i}^{\prime}\right) \varphi_{i}=1$.

We set

$$
\Psi=\omega^{*} \circ \omega_{\mathcal{M} *}: \mathcal{M}_{\zeta} \rightarrow \operatorname{Mod}\left(\mathcal{O}_{\mathcal{B}_{\zeta}}\right)
$$

Backelin and Kremnizer [2, Section 3.3] obtained the following result using a result of Artin and Zhang [1, Theorem 4.5]. 
Proposition 4.6. The functor $\Phi: \operatorname{Mod}\left(\mathcal{O}_{\mathcal{B}_{\zeta}}\right) \rightarrow \mathcal{M}_{\zeta}$ gives an equivalence of categories, and its quasi-inverse is given by $\Psi$. Moreover, we have an identification

$$
\omega_{\mathcal{M} *} \circ \Phi=\omega_{*}: \operatorname{Mod}\left(\mathcal{O}_{\mathcal{B}_{\zeta}}\right) \rightarrow \operatorname{Mod}_{\Lambda}\left(A_{\zeta}\right)
$$

of functors.

Hence we have the following.

LEMMA 4.7. We have

$$
R^{i} \Gamma=R^{i} \Gamma_{\mathcal{M}} \circ \Phi: \operatorname{Mod}\left(\mathcal{O}_{\mathcal{B}_{\zeta}}\right) \rightarrow \operatorname{Mod}(\mathbb{C})
$$

for any $i$.

We set

$$
\operatorname{Mod}^{\mathrm{eq}}\left(\mathcal{O}_{\mathcal{B}_{\zeta}}\right)=\operatorname{Mod}_{\Lambda}^{\mathrm{eq}}\left(A_{\zeta}\right) / \operatorname{Mod}_{\Lambda}^{\mathrm{eq}}\left(A_{\zeta}\right) \cap \operatorname{Tor}_{\Lambda^{+}}\left(A_{\zeta}\right)
$$

Let $N \in \operatorname{Mod}_{\Lambda}^{\mathrm{eq}}\left(A_{\zeta}\right)$. We denote the right $C_{\zeta}$-comodule structure of $N$ by $\gamma^{\prime}: N \rightarrow N \otimes C_{\zeta}$. Then we have a right $C_{\zeta^{-}}$comodule structure $\gamma: C_{\zeta} \otimes A_{\zeta}$ $N \rightarrow\left(C_{\zeta} \otimes_{A_{\zeta}} N\right) \otimes C_{\zeta}$ of $C_{\zeta} \otimes A_{\zeta} N$ given by

$$
\gamma^{\prime}(n)=\sum_{k} n_{k} \otimes \varphi_{k} \quad \Longrightarrow \quad \gamma(f \otimes n)=\sum_{k,(f)}\left(f_{(0)} \otimes n_{k}\right) \otimes f_{(1)} \varphi_{k} .
$$

This gives a functor $\operatorname{Mod}_{\Lambda}^{\mathrm{eq}}\left(A_{\zeta}\right) \rightarrow \mathcal{M}_{\zeta}^{\mathrm{eq}}$. Hence, by Lemma 4.5 we have a functor

$$
\Phi^{\mathrm{eq}}: \operatorname{Mod}^{\mathrm{eq}}\left(\mathcal{O}_{\mathcal{B}_{\zeta}}\right) \rightarrow \mathcal{M}_{\zeta}^{\mathrm{eq}}
$$

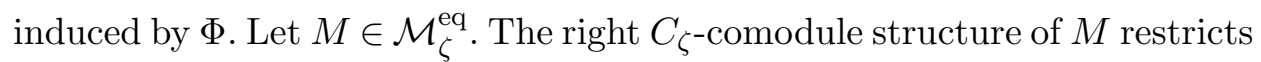
to that of $\omega_{\mathcal{M} *} M$ so that $\omega_{\mathcal{M} *} M \in \operatorname{Mod}_{\Lambda}^{\mathrm{eq}}\left(A_{\zeta}\right)$. Hence, we have a functor

$$
\Psi^{\mathrm{eq}}: \mathcal{M}_{\zeta}^{\mathrm{eq}} \rightarrow \operatorname{Mod}^{\mathrm{eq}}\left(\mathcal{O}_{\mathcal{B}_{\zeta}}\right)
$$

induced by $\Psi$. By Proposition 4.6, we have the following.

Proposition 4.8. The functor $\Phi^{\mathrm{eq}}: \operatorname{Mod}^{\mathrm{eq}}\left(\mathcal{O}_{\mathcal{B}_{\zeta}}\right) \rightarrow \mathcal{M}_{\zeta}^{\mathrm{eq}}$ gives an equivalence of categories, and its quasi-inverse is given by $\Psi^{\mathrm{eq}}$.

By Proposition 4.8 we see that (4.1) and (4.2) induce

$$
\begin{aligned}
& \omega_{*}^{\mathrm{eq}}=\omega_{\mathcal{M} *}^{\mathrm{eq}} \circ \Phi^{\mathrm{eq}}: \operatorname{Mod}^{\mathrm{eq}}\left(\mathcal{O}_{\mathcal{B}_{\zeta}}\right) \rightarrow \operatorname{Mod}_{\Lambda}^{\mathrm{eq}}\left(A_{\zeta}\right), \\
& \Gamma^{\mathrm{eq}}=\Gamma_{\mathcal{M}}^{\mathrm{eq}} \circ \Phi^{\mathrm{eq}}: \operatorname{Mod}^{\mathrm{eq}}\left(\mathcal{O}_{\mathcal{B}_{\zeta}}\right) \rightarrow \operatorname{Comod}^{r}\left(C_{\zeta}\right) .
\end{aligned}
$$

By Lemma 4.3, we have the following. 
LEMMA 4.9. We have

$$
\text { For } \circ R^{i} \Gamma^{\mathrm{eq}}=R^{i} \Gamma \circ \text { For }: \operatorname{Mod}^{\mathrm{eq}}\left(\mathcal{O}_{\mathcal{B}_{\zeta}}\right) \rightarrow \operatorname{Mod}(\mathbb{C})
$$

for any $i$, where For : $\operatorname{Comod}^{r}\left(C_{\zeta}\right) \rightarrow \operatorname{Mod}(\mathbb{C})$ and For $: \operatorname{Mod}^{\mathrm{eq}}\left(\mathcal{O}_{\mathcal{B}_{\zeta}}\right) \rightarrow$ $\operatorname{Mod}\left(\mathcal{O}_{\mathcal{B}_{\zeta}}\right)$ are forgetful functors.

\section{$\S 5$. Reformulation of Conjecture 2.14}

\subsection{Adjoint action of $U_{\zeta}^{L}$ on $D_{\zeta}^{\prime}$}

Define a left $U_{\mathbb{F}}$-module structure of $E_{\mathbb{F}}$ by

$$
\operatorname{ad}(u)(P)=\sum_{(u)} u_{(0)} P\left(S u_{(1)}\right) \quad\left(u \in U_{\mathbb{F}}, P \in E_{\mathbb{F}}\right) .
$$

Then we have

$$
\begin{aligned}
\operatorname{ad}(u)\left(P_{1} P_{2}\right) & =\sum_{(u)} \operatorname{ad}\left(u_{(0)}\right)\left(P_{1}\right) \operatorname{ad}\left(u_{(1)}\right)\left(P_{2}\right) \quad\left(P_{1}, P_{2} \in E_{\mathbb{F}}\right), \\
\operatorname{ad}(u)(\varphi) & =u \cdot \varphi \quad\left(\varphi \in A_{\mathbb{F}} \subset E_{\mathbb{F}}\right), \\
\operatorname{ad}(u)(v) & =\sum_{(u)} u_{(0)} v\left(S u_{(1)}\right) \quad\left(v \in U_{\mathbb{F}} \subset E_{\mathbb{F}}\right), \\
\operatorname{ad}(u)(e(\lambda)) & =\varepsilon(u) e(\lambda) \quad\left(\lambda \in \Lambda, e(\lambda) \in \mathbb{F}[\Lambda] \subset E_{\mathbb{F}}\right)
\end{aligned}
$$

for $u \in U_{\mathbb{F}}$. We see from [20, Lemma 4.2] that this induces a left $U_{\mathbb{F}}$-module structure of $D_{\mathbb{F}}^{\prime}$. Moreover, the $U_{\mathbb{F}}$-module structures of $E_{\mathbb{F}}$ and $D_{\mathbb{F}}^{\prime}$ induce $U_{\mathbb{A}}^{L}$-module structures of $E_{\mathbb{A}}, D_{\mathbb{A}}^{\prime}, E_{\mathbb{A}, \diamond}, D_{\mathbb{A}, \diamond}^{\prime}, E_{\mathbb{A}, f}$, and $D_{\mathbb{A}, f}^{\prime}$ by Lemmas 1.2 and 2.12. Hence, by specialization we obtain $U_{\zeta}^{L}$-module structures of $E_{\zeta}, D_{\zeta}^{\prime}, E_{\zeta, \diamond}, D_{\zeta, \diamond}^{\prime}, E_{\zeta, f}$, and $D_{\zeta, f}^{\prime}$ also denoted by ad.

\section{2 .}

We will regard $E_{\zeta, f}, D_{\zeta, f}^{\prime} \in \operatorname{Mod}_{\Lambda}\left(A_{\zeta}\right)$ as objects of $\operatorname{Mod}_{\Lambda}^{\mathrm{eq}}\left(A_{\zeta}\right)$ by the right $C_{\zeta}$-comodule structures induced from the left $U_{\zeta}^{L}$-module structures

$$
(u, P) \mapsto \operatorname{ad}(u)(P) \quad\left(u \in U_{\zeta}^{L}, P \in E_{\zeta, f} \text { or } D_{\zeta, f}^{\prime}\right) .
$$

Then for

$$
\left(\Xi \circ \Phi^{\mathrm{eq}}\right)\left(\omega^{*} D_{\zeta, f}^{\prime}\right) \in \operatorname{Comod}\left(C_{\zeta}^{\leqq 0}\right)
$$

we have

$$
R^{i} \Gamma\left(\omega^{*} D_{\zeta, f}^{\prime}\right)=R^{i} \operatorname{Ind}\left(\left(\Xi \circ \Phi^{\mathrm{eq}}\right)\left(\omega^{*} D_{\zeta, f}^{\prime}\right)\right)
$$

by Lemmas 4.3 and 4.9 and by (4.10). 
Define a right $\left(U_{\zeta, \diamond} \otimes \mathbb{C}[\Lambda]\right)$-module $V$ by

$$
V=\left(U_{\zeta, \diamond} \otimes \mathbb{C}[\Lambda]\right) / \mathcal{I}
$$

where

$$
\mathcal{I}=\left(\tilde{U}_{\zeta}^{-} \cap \operatorname{Ker}(\varepsilon)\right) U_{\zeta, \diamond} \mathbb{C}[\Lambda]+\sum_{\lambda \in \Lambda}\left(k_{2 \lambda}-e(2 \lambda)\right) U_{\zeta, \diamond} \mathbb{C}[\Lambda]
$$

By the triangular decomposition $\tilde{U}_{\zeta}^{-} \otimes U_{\zeta, \diamond}^{0} \otimes U_{\zeta}^{+} \cong U_{\zeta, \diamond}$ we have

$$
V \cong U_{\zeta}^{+} \otimes \mathbb{C}[\Lambda]
$$

as a vector space. Define a right action of $U_{\zeta}^{L, \leqq 0}$ on $U_{\zeta, \diamond} \otimes \mathbb{C}[\Lambda]$ by

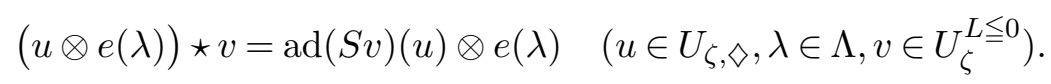

It induces a right action of $U_{\zeta}^{L, \leqq 0}$ on $V$. Moreover, we see easily that this right $U_{\zeta}^{L, \leqq 0}$-module structure gives a left $C_{\zeta}^{\leqq 0}$-comodule structure of $V$.

Proposition 5.1. We have

$$
\left(\Xi \circ \Phi^{\mathrm{eq}}\right)\left(\omega^{*} D_{\zeta, f}^{\prime}\right) \cong V
$$

as a left $C_{\zeta}^{\leqq 0}$-comodule.

The proof is given in Section 5.3.

It follows from Proposition 5.1 that Conjecture 2.14 is equivalent to the following conjecture.

Conjecture 5.2. Assume that $\ell>h_{G}$. We have

$$
\operatorname{Ind}(V) \cong U_{\zeta, f} \otimes_{Z_{\mathrm{Har}}\left(U_{\zeta}\right)} \mathbb{C}[\Lambda]
$$

and

$$
R^{i} \operatorname{Ind}(V)=0
$$

for $i \neq 0$.

REMARK 5.3. We can show that

$$
U_{\zeta, f} \cong\left(C_{\zeta}\right)_{\mathrm{ad}}, \quad V \cong{ }_{\mathrm{ad}}\left(C_{\bar{\zeta}}^{\leqq 0}\right) \otimes_{\mathbb{C}[2 \Lambda]} \mathbb{C}[\Lambda],
$$


where $\left(C_{\zeta}\right)_{\text {ad }}$ (resp., ad $\left.\left(C_{\zeta}^{\leqq 0}\right)\right)$ is given by the right (resp., left) adjoint coaction of $C_{\zeta}$ (resp., $C_{\bar{\zeta}}^{\leqq 0}$ ) on itself. Hence, Conjecture 5.2 is equivalent to

$$
R \operatorname{Ind}\left({ }_{\operatorname{ad}}\left(C_{\zeta}^{\leqq 0}\right)\right) \cong\left(C_{\zeta}\right)_{\mathrm{ad}} \otimes_{\mathbb{C}[2 \Lambda]^{W}} \mathbb{C}[2 \Lambda] .
$$

The corresponding statement for $q=1$ is

$$
R \operatorname{Ind}\left(\mathrm{ad} \mathbb{C}\left[B^{-}\right]\right) \cong \mathbb{C}[G]_{\mathrm{ad}} \otimes_{\mathbb{C}[H / W]} \mathbb{C}[H] .
$$

We can prove this by a geometric method.

REMARK 5.4. ${ }^{\dagger}$ A proof of Conjecture 5.2, when $\ell$ is a prime greater than the Coxeter number, is given by Backelin and Kremnizer in [3, Proposition 3.25]; however, in a more recent article they admit that there are gaps in [3] (see [4, Version 3, Section 1.1.2]) and propose different proofs. But it is likely that problems still remain in the new proofs given in [4], as explained below.

The proof in [4, Versions 1 and 2] is wrong because all positive roots are assumed there to be dominant (see [4, Version 2, proof of Theorem 2.1]).

Another proof given in [4, Version 3] also has problems. In Step (b) of [4, Version 3, proof of Theorem 2.2.1], the authors compare certain weight multiplicities $a_{q, \mu}$ and $b_{q, \mu}$. But since those multiplicities are infinite, the argument there should be modified using multiplicities as $U_{q}$-modules. Let us assume for simplicity that $q$ is generic and try to modify the original argument by replacing $a_{q, \mu}, b_{q, \mu}, b_{q, \mu}^{\prime}$ with their counterparts as multiplicities of $U_{q}$-modules. This even fails since $a_{1, \mu}$ (resp., $b_{1, \mu}^{\prime}$ ) is the dimension of the 0 -weight space of the irreducible module (resp., Verma module) with highest-weight $\mu$. We also point out that the reason that $U_{q}^{\lambda}$ is an integral domain is not given in Step (a).

Note that the arguments in [4, Version 3, proof of Theorem 2.2.1] are partially similar to those in the earlier manuscripts (see [2, Proposition 4.8], [3, Proposition 3.25]). The main difference is that [4, Version 3] relies on a $B_{q}$-stable filtration with 1-dimensional subquotients instead of the JosephLetzter filtration used in [2] and [3]. For us, the original argument in [2] and [3] for generic $q$ using the Joseph-Letzter filtration is not comprehensible either. In the notation of [2, proof of Proposition 4.8], the validity of the formula $m_{j}(1)=\tilde{n}_{j}(1)$ is not clear to us since the Joseph-Letzter filtration does not induce at $q=1$ the ordinary filtration for enveloping algebras and differential operators in general.

\footnotetext{
†This remark is added at the editor's request.
} 


\section{3.}

We will give a proof of Proposition 5.1 in the rest of this article. By Remark 4.2, we have

$$
\left(\Xi \circ \Phi^{\mathrm{eq}}\right)\left(\omega^{*} D_{\zeta, f}^{\prime}\right) \cong \mathbb{C} \otimes_{A_{\zeta}} D_{\zeta, f}^{\prime}
$$

as a vector space, where $A_{\zeta} \rightarrow \mathbb{C}$ is given by $\varepsilon$. Note that

$$
\mathbb{C} \otimes A_{\zeta} E_{\zeta, \diamond} \cong U_{\zeta, \diamond} \otimes \mathbb{C}[\Lambda]
$$

We first show the following.

LEMMA 5.5. We have

$$
\mathbb{C} \otimes_{A_{\zeta}} D_{\zeta, \diamond}^{\prime} \cong V
$$

Proof. By (2.10) we obtain

$$
\mathbb{C} \otimes A_{\zeta} D_{\zeta, \diamond}^{\prime} \cong\left(U_{\zeta, \diamond} \otimes \mathbb{C}[\Lambda]\right) / \sum_{\varphi \in A_{\zeta}}\left(1 \otimes \Omega^{\prime}(\varphi)\right)\left(U_{\zeta, \diamond} \otimes \mathbb{C}[\Lambda]\right),
$$

where $1 \otimes \Omega^{\prime}(\varphi)$ is the image of $\Omega^{\prime}(\varphi)$ in $\mathbb{C} \otimes_{A_{\zeta}} E_{\zeta, \diamond}=U_{\zeta, \diamond} \otimes \mathbb{C}[\Lambda]$. Note that $\varepsilon\left(A_{\zeta}(\lambda)_{\xi}\right)=\{0\}$ for $\lambda \in \Lambda^{+}, \xi \in \Lambda$ with $\lambda \neq \xi$, and that $\varepsilon\left(A_{\zeta}(\lambda)_{\lambda}\right)=\mathbb{C}$ for $\lambda \in \Lambda^{+}$. Hence, for $\varphi \in A_{\zeta}(\lambda)_{\xi}$ with $\lambda \in \Lambda^{+}, \xi \in \Lambda$ we have

$$
1 \otimes \Omega_{1}^{\prime}(\varphi)= \begin{cases}0 & (\lambda \neq \xi), \\ \varepsilon(\varphi) & (\lambda=\xi) .\end{cases}
$$

Let us also compute $1 \otimes \Omega_{2}^{\prime}(\varphi)$. Let

$$
\tilde{\Psi}_{\lambda}: \tilde{U}_{\zeta}^{-}(\lambda) \rightarrow A_{\zeta}(\lambda)
$$

be the composite of the linear isomorphism $\Psi_{\lambda}: \tilde{U}_{\zeta}^{-}(\lambda) \rightarrow L_{-, \zeta}^{*}(\lambda)$ (see (3.33)) and an isomorphism $f: L_{-, \zeta}^{*}(\lambda) \rightarrow A_{\zeta}(\lambda)$ of $U_{\zeta}^{L}$-modules. We have $\tilde{\Psi}_{\lambda}\left(\tilde{U}_{\zeta}^{-}(\lambda)_{-(\lambda-\xi)}\right)=A_{\zeta}(\lambda)_{\xi}$ for any $\xi \in \Lambda$. Hence, we may assume that $\varepsilon=$ $\varepsilon \circ \tilde{\Psi}_{\lambda}$ on $\tilde{U}_{\zeta}^{-}(\lambda)$. Let $\varphi \in A_{\zeta}(\lambda)_{\xi}$, and take $v \in \tilde{U}_{\zeta}^{-}(\lambda)_{-(\lambda-\xi)}$ satisfying $\tilde{\Psi}_{\lambda}(v)=\varphi$. Then we have

$$
\begin{aligned}
\sum_{p}\left(S x_{p}^{L}\right) \cdot \varphi \otimes y_{p} k_{\beta_{p}} & =\sum_{p} f\left(\left(S x_{p}^{L}\right) \cdot \Psi_{\lambda}(v)\right) \otimes y_{p} k_{\beta_{p}} \\
& =\sum_{p} \zeta^{-\left(\beta_{p}, \xi\right)} f\left(\left(S x_{p}^{L}\right) k_{\beta_{p}} \cdot \Psi_{\lambda}(v)\right) \otimes y_{p} k_{\beta_{p}}
\end{aligned}
$$




$$
\begin{aligned}
& =\sum_{p,(v)} \zeta^{-\left(\beta_{p}, \xi\right)} f\left({ }^{L} \tau_{\zeta}\left(\left(S x_{p}^{L}\right) k_{\beta_{p}}, v_{(0)}\right) \Psi_{\lambda}\left(v_{(1)}\right)\right) \otimes y_{p} k_{\beta_{p}} \\
& =\sum_{p,(v)} \zeta^{-\left(\beta_{p}, \xi\right) L} \tau_{\zeta}\left(\left(S x_{p}^{L}\right) k_{\beta_{p}}, v_{(0)}\right) \tilde{\Psi}_{\lambda}\left(v_{(1)}\right) \otimes y_{p} k_{\beta_{p}}
\end{aligned}
$$

and hence

$$
\begin{aligned}
1 \otimes \Omega_{2}^{\prime}(\varphi) & =\sum_{p} \varepsilon\left(\left(S x_{p}^{L}\right) \cdot \varphi\right) y_{p} k_{\beta_{p}} k_{2 \xi} e(-2 \lambda) \\
& =\sum_{p,(v)} \zeta^{-\left(\beta_{p}, \xi\right) L} \tau_{\zeta}\left(\left(S x_{p}^{L}\right) k_{\beta_{p}}, v_{(0)}\right) \varepsilon\left(v_{(1)}\right) y_{p} k_{\beta_{p}} k_{2 \xi} e(-2 \lambda) \\
& =\sum_{p} \zeta^{-\left(\beta_{p}, \xi\right) L} \tau_{\zeta}\left(\left(S x_{p}^{L}\right) k_{\beta_{p}}, v\right) y_{p} k_{\beta_{p}} k_{2 \xi} e(-2 \lambda) \\
& =\sum_{p} \zeta^{-\left(\beta_{p}, \xi\right) L} \tau_{\zeta}\left(k_{-\beta_{p}} x_{p}^{L}, S^{-1} v\right) y_{p} k_{\beta_{p}} k_{2 \xi} e(-2 \lambda) \\
& =\sum_{p} \zeta^{-\left(\beta_{p}, \xi\right)-\left(\beta_{p}, \beta_{p}\right) L} \tau_{\zeta}\left(x_{p}^{L}, S^{-1} v\right) y_{p} k_{\beta_{p}} k_{2 \xi} e(-2 \lambda) \\
& =\sum_{p} \zeta^{-(\lambda-\xi, \lambda) L} \tau_{\zeta}\left(x_{p}^{L}, S^{-1} v\right) y_{p} k_{\lambda-\xi} k_{2 \xi} e(-2 \lambda) \\
& =\zeta^{-(\lambda-\xi, \lambda)}\left(S^{-1} v\right) k_{\lambda-\xi} k_{2 \xi} e(-2 \lambda) .
\end{aligned}
$$

(Note that $\left.\left(S^{-1} v\right) k_{\lambda-\xi} \in \tilde{U}_{\zeta}^{-}(\lambda)_{-(\lambda-\xi)}.\right)$ It follows that

$$
1 \otimes \Omega^{\prime}(\varphi)= \begin{cases}-\zeta^{-(\lambda-\xi, \lambda)}\left(S^{-1} v\right) k_{\lambda-\xi} k_{2 \xi} e(-2 \lambda) & (\lambda \neq \xi), \\ \varepsilon(\varphi)\left(1-k_{2 \lambda} e(-2 \lambda)\right) & (\lambda=\xi) .\end{cases}
$$

Hence, we have

$$
\begin{aligned}
& \sum_{\substack{\lambda \in \Lambda^{+}, \varphi \in A_{\zeta}(\lambda)_{\lambda-\gamma} \\
\gamma \in Q^{+}}} \sum_{\substack{\lambda \in \Lambda^{+}, \gamma \in Q^{+} \backslash\{0\}}} \tilde{U}_{\zeta}^{-}(\lambda)_{-\gamma}\left(U_{\zeta, \diamond} \otimes \mathbb{C}[\Lambda]\right)+\sum_{\lambda \in \Lambda^{+}}\left(1-k_{2 \lambda} e(-2 \lambda)\right)\left(U_{\zeta, \diamond} \otimes \mathbb{C}[\Lambda]\right) \\
& =\left(\tilde{U}_{\zeta}^{-} \cap \operatorname{Ker}(\varepsilon)\right)\left(U_{\zeta, \diamond} \otimes \mathbb{C}[\Lambda]\right)+\sum_{\lambda \in \Lambda}\left(k_{2 \lambda}-e(2 \lambda)\right)\left(U_{\zeta, \diamond} \otimes \mathbb{C}[\Lambda]\right)
\end{aligned}
$$

by $(3.35)$. 
LEMMA 5.6. We have

$$
\mathbb{C} \otimes_{A_{\zeta}} D_{\zeta, f}^{\prime} \cong V
$$

Proof. We need to show that the canonical homomorphism $\mathbb{C} \otimes_{A_{\zeta}} D_{\zeta, f}^{\prime} \rightarrow$ $\mathbb{C} \otimes A_{\zeta} D_{\zeta, \diamond}^{\prime}$ is bijective. The surjectivity is a consequence of (3.35) and (3.36). Let us give a proof of the injectivity. Set

$$
\mathcal{K}=A_{\zeta} U_{\zeta, f} \mathbb{C}[\Lambda] \cap \sum_{\varphi \in A_{\zeta}} A_{\zeta} \Omega^{\prime}(\varphi) U_{\zeta, \diamond} \mathbb{C}[\Lambda] \subset A_{\zeta} \otimes U_{\zeta, f} \otimes \mathbb{C}[\Lambda] .
$$

Then it is sufficient to show that the natural map

$$
\mathbb{C} \otimes A_{\zeta}\left(\left(A_{\zeta} \otimes U_{\zeta, f} \otimes \mathbb{C}[\Lambda]\right) / \mathcal{K}\right) \rightarrow\left(U_{\zeta, \diamond} \otimes \mathbb{C}[\Lambda]\right) / \mathcal{I}
$$

is injective. Let $F: A_{\zeta} \otimes U_{\zeta, f} \otimes \mathbb{C}[\Lambda] \rightarrow U_{\zeta, \diamond} \otimes \mathbb{C}[\Lambda]$ be the natural map. Then it is sufficient to show that

$$
\mathcal{I} \cap\left(U_{\zeta, f} \otimes \mathbb{C}[\Lambda]\right) \subset F(\mathcal{K})
$$

Indeed, assume that (5.1) holds. Denote by

$$
\begin{gathered}
p: A_{\zeta} \otimes U_{\zeta, f} \otimes \mathbb{C}[\Lambda] \rightarrow \mathbb{C} \otimes A_{\zeta}\left(\left(A_{\zeta} \otimes U_{\zeta, f} \otimes \mathbb{C}[\Lambda]\right) / \mathcal{K}\right) \\
\pi: U_{\zeta, \diamond} \otimes \mathbb{C}[\Lambda] \rightarrow\left(U_{\zeta, \diamond} \otimes \mathbb{C}[\Lambda]\right) / \mathcal{I}
\end{gathered}
$$

the natural maps. We have to show that $\operatorname{Ker}(\pi \circ F) \subset \operatorname{Ker}(p)$. Take $x \in$ $\operatorname{Ker}(\pi \circ F)$. Then $F(x) \in \mathcal{I} \cap\left(U_{\zeta, f} \otimes \mathbb{C}[\Lambda]\right)$. Hence, by (5.1) there exists some $v \in \mathcal{K}$ such that $F(x)=F(v)$. Then $p(x)=p(x-v)+p(v)=p(x-v)$. Hence, we may assume that $F(x)=0$ from the beginning. Note that $p$ factors through

$$
p^{\prime}: A_{\zeta} \otimes U_{\zeta, f} \otimes \mathbb{C}[\Lambda] \rightarrow \mathbb{C} \otimes A_{\zeta}\left(A_{\zeta} \otimes U_{\zeta, f} \otimes \mathbb{C}[\Lambda]\right)\left(=U_{\zeta, f} \otimes \mathbb{C}[\Lambda]\right) .
$$

By $F(x)=0$ we have $p^{\prime}(x)=0$, and hence $p(x)=0$, as desired.

It remains to show (5.1). Let $\lambda \in \Lambda^{+}$, and let $\varphi \in A_{\zeta}(\lambda)_{\lambda}$. Then we have

$$
\Omega_{1}^{\prime}(\varphi)=\sum_{p}\left(y_{p}^{L} \cdot \varphi\right) x_{p} \in A_{\zeta} U_{\zeta}^{+}, \quad \Omega_{2}^{\prime}(\varphi)=\varphi k_{2 \lambda} e(-2 \lambda) .
$$

Let us show that

$$
\Omega_{1}^{\prime}(\varphi)=\sum_{p}\left(y_{p}^{L} \cdot \varphi\right) x_{p} \in A_{\zeta} U_{\zeta}^{+}(\lambda)
$$


This is equivalent to

$$
\sum_{p}\left(y_{p}^{L} \cdot \varphi\right) \otimes \Phi_{-\lambda}\left(x_{p}\right) \in A_{\zeta} \otimes L_{+, \zeta}^{*}(-\lambda) .
$$

This follows from

$$
\begin{aligned}
\sum_{p}\left\langle\Phi_{-\lambda}\left(x_{p}\right), \overline{u f_{i}^{\left(\left(\lambda, \alpha_{i}^{\vee}\right)+1\right)}}\right\rangle y_{p}^{L} \cdot \varphi & =\sum_{p} \tau_{\zeta}^{L}\left(x_{p}, u f_{i}^{\left(\left(\lambda, \alpha_{i}^{\vee}\right)+1\right)}\right) y_{p}^{L} \cdot \varphi \\
& =\left(u f_{i}^{\left(\left(\lambda, \alpha_{i}^{\vee}\right)+1\right)}\right) \cdot \varphi=0
\end{aligned}
$$

for $u \in U_{\zeta}^{L,-}, i \in I$. Thus, (5.2) is verified. Hence, we have

$$
\Omega^{\prime}(\varphi) k_{-2 \lambda} \in \mathcal{K}
$$

It follows that

$$
F(\mathcal{K}) \supset\left(k_{-2 \lambda}-e(-2 \lambda)\right) U_{\zeta, f} \mathbb{C}[\Lambda] \quad\left(\lambda \in \Lambda^{+}\right)
$$

Now let $u \in \mathcal{I} \cap\left(U_{\zeta, f} \otimes \mathbb{C}[\Lambda]\right)$. If we can show that $k_{-2 \mu} u \in F(\mathcal{K})$ for some $\mu \in \Lambda^{+}$, then we obtain

$$
u=e(2 \mu)\left(e(-2 \mu)-k_{-2 \mu}\right) u+e(2 \mu) k_{-2 \mu} u \in F(\mathcal{K})
$$

by (5.3). Hence, it is sufficient to show that for any $u \in \mathcal{I}$ there exists some $\mu \in \Lambda^{+}$such that $k_{-2 \mu} u \in F(\mathcal{K})$. We may assume that there exists $\nu \in Q$ such that $k_{-2 \mu} u=\zeta^{(\mu, \nu)} u k_{-2 \mu}$ for any $\mu \in \Lambda$. Therefore, we have only to show that for any $u \in \mathcal{I}$ there exists some $\mu \in \Lambda^{+}$such that $u k_{-2 \mu} \in F(\mathcal{K})$. By Lemma 5.5 we can take $\varphi_{i} \in A_{\zeta}, x_{i} \in U_{\zeta, \diamond} \otimes \mathbb{C}[\Lambda](i=1, \ldots, N)$ such that

$$
u=1 \otimes \sum_{i=1}^{N} \Omega^{\prime}\left(\varphi_{i}\right) x_{i}
$$

By Lemma 3.7 we can take $\mu \in \Lambda^{+}$such that $\Omega^{\prime}\left(\varphi_{i}\right) x_{i} k_{-2 \mu} \in A_{\zeta} \otimes U_{\zeta, f} \otimes \mathbb{C}[\Lambda]$ for any $i$. Then we have

$$
u k_{-2 \mu}=\sum_{i=1}^{N} F\left(\Omega^{\prime}\left(\varphi_{i}\right) x_{i} k_{-2 \mu}\right) \in F(\mathcal{K}) .
$$


By Lemma 5.6 we obtain an isomorphism

$$
\left(\Xi \circ \Phi^{\mathrm{eq}}\right)\left(\omega^{*} D_{\zeta, f}^{\prime}\right) \cong V
$$

of vector spaces. We need to show that it is in fact an isomorphism of left $C_{\bar{\zeta}}^{\leqq 0}$-comodules. This is a consequence of the corresponding fact for $E_{\zeta, f}$. Note that we have

$$
\mathbb{C} \otimes A_{\zeta} E_{\zeta, f} \cong U_{\zeta, f} \otimes \mathbb{C}[\Lambda]
$$

and hence we have an isomorphism

$$
\left(\Xi \circ \Phi^{\mathrm{eq}}\right)\left(\omega^{*} E_{\zeta, f}\right) \cong U_{\zeta, f} \otimes \mathbb{C}[\Lambda]
$$

of vector spaces. Hence, we have only to show the following.

LEMMA 5.7. Under identification (5.4), the left $C_{\bar{\zeta}}^{\leqq 0}$-comodule structure of $U_{\zeta, f} \otimes \mathbb{C}[\Lambda]$ is associated to the right $U_{\zeta}^{L, \leqq 0}{ }^{-m o d u l e}$ structure given by

$$
(u \otimes e(\lambda)) \cdot v=\operatorname{ad}(S v)(u) \otimes e(\lambda) \quad\left(u \in U_{\zeta, f}, \lambda \in \Lambda, v \in U_{\zeta}^{L, \leqq 0}\right) .
$$
by

Proof. Note that the left $C_{\zeta}^{\leq 0}$-comodule structure of $U_{\zeta, f} \otimes \mathbb{C}[\Lambda]$ is given

$$
U_{\zeta, f} \otimes \mathbb{C}[\Lambda] \cong \Xi\left(C_{\zeta} \otimes\left(U_{\zeta, f} \otimes \mathbb{C}[\Lambda]\right)\right)
$$

where $C_{\zeta} \otimes\left(U_{\zeta, f} \otimes \mathbb{C}[\Lambda]\right)$ is regarded as a left $C_{\zeta}^{\leqq 0}$-comodule by the tensor product of $C_{\zeta}$ (with left $C_{\zeta}^{\leqq 0}$-comodule structure (res $\left.\otimes 1\right) \circ \Delta: C_{\zeta} \rightarrow C_{\zeta}^{\leqq 0} \otimes$ $\left.C_{\zeta}\right)$ and $U_{\zeta, f} \otimes \mathbb{C}[\Lambda]$ with trivial left $C_{\bar{\zeta}}^{\leqq 0}$-comodule structure. Hence, it is sufficient to show that for a right $C_{\zeta^{-}}$comodule $M$ the right $U_{\zeta}^{L, \leqq 0}$-module structure of

$$
M \cong \Xi\left(C_{\zeta} \otimes M\right) \in \operatorname{Comod}\left(C_{\zeta}^{\leqq 0}\right)
$$

is given by

$$
m \cdot v=(S v) \cdot m \quad\left(m \in M, v \in U_{\zeta}^{L, \leqq 0}\right) .
$$

Denote by $M^{\text {triv }}$ the trivial right $C_{\zeta}$-comodule which coincides with $M$ as a vector space. We denote by $M \ni m \leftrightarrow \bar{m} \in M^{\text {triv }}$ the canonical linear isomorphism. We have $C_{\zeta} \otimes M^{\text {triv }} \in \operatorname{Comod}^{r}\left(C_{\zeta}\right)$ as the tensor product of $C_{\zeta} \in \operatorname{Comod}^{r}\left(C_{\zeta}\right)$ and $M^{\text {triv }} \in \operatorname{Comod}^{r}\left(C_{\zeta}\right)$. We can also define a left $C_{\zeta}^{\leq 0}$-comodule structure of $C_{\zeta} \otimes M^{\text {triv }}$ as the tensor product of the left 
$C_{\bar{\zeta}}^{\leqq 0}$-comodules $C_{\zeta}$ and $M^{\text {triv }}$, where the left $C_{\bar{\zeta}}^{\leqq 0}$-comodule structure of $M^{\text {triv }}$ is given by the right $U_{\zeta}^{L, \leqq 0}$-module structure

$$
\bar{m} \cdot v=\overline{(S v) \cdot m} \quad\left(m \in M, v \in U_{\zeta}^{L, \leqq 0}\right) .
$$

Then we have a linear isomorphism

$$
C_{\zeta} \otimes M \ni \varphi \otimes m \mapsto \sum_{(m)} \varphi m_{(1)} \otimes \overline{m_{(0)}} \in C_{\zeta} \otimes M^{\text {triv }}
$$

preserving the right $C_{\zeta}$-comodule structures and the left $C_{\zeta}^{\leqq 0}$-comodule structures. It follows that

$$
\Xi\left(C_{\zeta} \otimes M\right) \cong \Xi\left(C_{\zeta} \otimes M^{\text {triv }}\right)=M^{\text {triv }} \in \operatorname{Comod}\left(C_{\zeta}^{\leqq 0}\right) .
$$

The proof of Proposition 5.1 is complete.

\section{REFERENCES}

[1] M. Artin and J. J. Zhang, Noncommutative projective schemes, Adv. Math. 109 (1994), 228-287. MR 1304753. DOI 10.1006/aima.1994.1087.

[2] E. Backelin and K. Kremnizer, Quantum flag varieties, equivariant quantum $\mathcal{D}$ modules, and localization of quantum groups, Adv. Math. 203 (2006), 408-429. MR 2227727. DOI 10.1016/j.aim.2005.04.012.

[3] - Localization for quantum groups at a root of unity, J. Amer. Math. Soc. 21 (2008), 1001-1018. MR 2425178. DOI 10.1090/S0894-0347-08-00608-5.

[4] - Global quantum differential operators on quantum flag manifolds, theorems of Duflo and Kostant, preprint, arXiv:1106.4343 [math.RT].

[5] R. Bezrukavnikov, I. Mirković, and D. Rumynin, Singular localization and intertwining functors for reductive Lie algebras in prime characteristic, Nagoya Math. J. 184 (2006), 1-55. MR 2285230.

[6] - Localization of modules for a semisimple Lie algebra in prime characteristic, Ann. of Math. (2) 167 (2008), 945-991. MR 2415389. DOI 10.4007/annals.2008.167. 945.

[7] K. A. Brown and K. R. Goodearl, Homological aspects of Noetherian PI Hopf algebras of irreducible modules and maximal dimension, J. Algebra 198, (1997), 240-265. MR 1482982. DOI 10.1006/jabr.1997.7109.

[8] C. De Concini and V. G. Kac, "Representations of quantum groups at roots of 1" in Operator Algebras, Unitary Representations, Enveloping Algebras, and Invariant Theory (Paris, 1989), Progr. Math. 92, Birkhäuser, Boston, 1990, 471-506. MR 1103601.

[9] C. De Concini and C. Procesi, "Quantum groups" in D-modules, Representation Theory and Quantum Groups (Venice, 1992), Lecture Notes in Math. 1565, Springer, Berlin, 1993, 31-140. MR 1288995. DOI 10.1007/BFb0073466.

[10] F. Gavarini, Quantization of Poisson groups, Pacific J. Math. 186 (1998), 217-266. MR 1663802. DOI 10.2140/pjm.1998.186.217. 
[11] A. Joseph, Quantum Groups and Their Primitive Ideals, Ergeb. Math. Grenzgeb. (3) 29, Springer, Berlin, 1995. MR 1315966.

[12] A. Joseph and G. Letzter, Separation of variables for quantized enveloping algebras, Amer. J. Math. 116 (1994), 127-177. MR 1262429. DOI 10.2307/2374984.

[13] G. Lusztig, Quantum deformations of certain simple modules over enveloping algebras, Adv. Math. 70 (1988), 237-249. MR 0954661. DOI 10.1016/0001-8708(88) 90056-4.

[14] - Quantum groups at roots of 1, Geom. Dedicata 35 (1990), 89-113. MR 1066560. DOI 10.1007/BF00147341.

[15] - Introduction to Quantum Groups, Progr. Math. 110, Birkhäuser, Boston, 1993. MR 1227098.

[16] N. Popescu, Abelian Categories with Applications to Rings and Modules, London Math. Soc. Monogr. Ser. 3, Academic Press, London, 1973. MR 0340375.

[17] R. Steinberg, On a theorem of Pittie, Topology 14 (1975), 173-177. MR 0372897.

[18] T. Tanisaki, "Killing forms, Harish-Chandra isomorphisms, and universal $R$-matrices for quantum algebras" in Infinite Analysis, Part A, B (Kyoto, 1991), Adv. Ser. Math. Phys. 16, World Sci. Publ., River Edge, New Jersey, 1992, 941-961. MR 1187582.

[19] - The Beilinson-Bernstein correspondence for quantized enveloping algebras, Math. Z. 250 (2005), 299-361. MR 2178788. DOI 10.1007/s00209-004-0754-9.

[20] - Differential operators on quantized flag manifolds at roots of unity, Adv. Math. 230 (2012), 2235-2294. MR 2927370. DOI 10.1016/j.aim.2012.04.018.

[21] - Manin triples and differential operators on quantum groups, Tokyo J. Math., 36 (2013), 49-83.

Department of Mathematics

Osaka City University

Sumiyoshi-ku, Osaka

558-8585 Japan

tanisaki@sci.osaka-cu.ac.jp 